\title{
Joint Annual Meeting of the
}

Swiss Respiratory Society

Swiss Society of Oto-Rhino-Laryngology, Head and Neck Surgery

Swiss Paediatric Respiratory Society

Swiss Society for Thoracic Surgery

Interlaken, May 4-6, 2011

\section{Free Communications 1 Clinical Research: COPD/Asthma}

23

Polysomnography Under Non-invasive Ventilation in Stable COPD to Reduce Patient-ventilator Asynchrony and Morning Breathlessness

D. Adler ${ }^{1}$, S. Perrig ${ }^{1}$, H. Takahashi ${ }^{1}$, F. Espa ${ }^{1}$, D. Rodenstein ${ }^{2}$, J.-L. Pépin ${ }^{3}$, J.-P. Janssens ${ }^{1}$

${ }^{1}$ HUG, Genève, ${ }^{2}$ Catholic University of Louvain, Woluwe, $B$, 3INSERM U 1042, Grenoble, F

Background: Patient-ventilator asynchrony (PVA) is frequent in patients undergoing non-invasive ventilation (NIV) who are considered effectively treated. Pulsoximetry and transcutaneous capnography $\left(\mathrm{TcPCO}_{2}\right)$ monitoring cannot exclude PVA. We hypothesized that home NIV in severe COPD with inadequate settings may lead to hyperinflation with increased PEEPi and result in unrewarded efforts and morning dyspnea when interrupting NIV. Adjusting ventilator settings during polysomnography (PSG) might improve sleep quality, patient-ventilator synchronization and morning dyspnea ('deventilation dyspnea').

Methods: 8 consecutive severe COPD patients, scheduled for a routine control of NIV, were admitted at Geneva University hospital sleep lab for two nights. PSG under NIV was recorded with the patient's usual ventilator settings on the first night. Ventilator settings were adjusted using on-line PSG with $\mathrm{TcPCO}_{2} / \mathrm{SaO}_{2}$ monitoring in order to reduce PVA on the second night.

Results: Pressure support reduction (IPAP-EPAP) in all cases (baseline: $13.6 \pm 1.8 \mathrm{~cm} \mathrm{H}_{2} \mathrm{O}$; adjusted: $10.3 \pm 1.7 \mathrm{~cm} \mathrm{H}_{2} \mathrm{O}, \mathrm{p}=.0005$ ) resulted in reduction of PVA index from $40.5 \% \pm 31.0$ to $6.7 \% \pm 7.3$, $\mathrm{p}=.009$ (fig. 1). Total sleep time, microarousal index and sleep efficiency were not significantly changed. Pressure support reduction resulted in a marked decrease in morning 'deventilation dyspnea' measured by a modified Borg scale (baseline: $5.8 \pm 2.5$; adjusted: $2.3 \pm 1.6, p=.0059$ ). Subjective assessment of ventilation using a visual analog scale showed improvement of perceived PVA $(\mathrm{p}=.04)$, perception of leaks $(\mathrm{p}=.04)$ and overall quality of sleep $(\mathrm{p}=.01)$ (fig. 2). Pressure support reduction did not increase mean nocturnal $\mathrm{TcPO}_{2}$ (baseline: $7.5 \pm 1.2 \mathrm{kPa}$; adjusted: $7.3 \pm 1.5 \mathrm{kPa}$, $\mathrm{p}=.06)$.

Conclusion: This study confirms that a very high rate of PVA in severe COPD under home ventilation can remain undetected without PSG. Adjusting ventilator settings using online PSG resulted in: 1) improved patient ventilator synchronization; 2) improved patient comfort; 3) decreased 'deventilation dyspnea'; 4) without any deterioration in mean TcPCO2.

24

Improvement of Quality of Life in Mild to Moderate COPD After Treatment with Inhaled Corticosteroids Based on Airway Responsiveness to Mannitol

A. Scherr, A. Jochmann, S. Schaffroth, D. Miedinger, S. Maier, M. Tamm, D. Leuppi

University of Basel, Basel

Background: The effect of inhaled corticosteroids (ICS) is controversially discussed in mild to moderate COPD. Based on former data airway hyperresponsiveness (AHR) to mannitol challenge could allow to identify a subgroup of patients who will respond to treatment with ICS.

Methods: We investigated 68 subjects (61.5 years (range 36-84)) with mild to moderately severe COPD. All subjects had postbronchodilator FEV1/FVC of less than $70 \%$, FEV1> $50 \%$ and a reversibility of less than $12 \%$ or $200 \mathrm{ml}$ from baseline in spirom-

\begin{tabular}{ll}
\hline KARGER & ( ) 2011 S. Karger AG, Basel \\
0025-7931/11/0821-0067\$38.00/0 \\
$\begin{array}{l}\text { Fax +4161306 12 34 } \\
\begin{array}{l}\text { E-Mail karger@karger.ch } \\
\text { www.karger.com }\end{array}\end{array}$ & $\begin{array}{l}\text { Accessible online at: } \\
\text { www.karger.com/res }\end{array}$
\end{tabular}




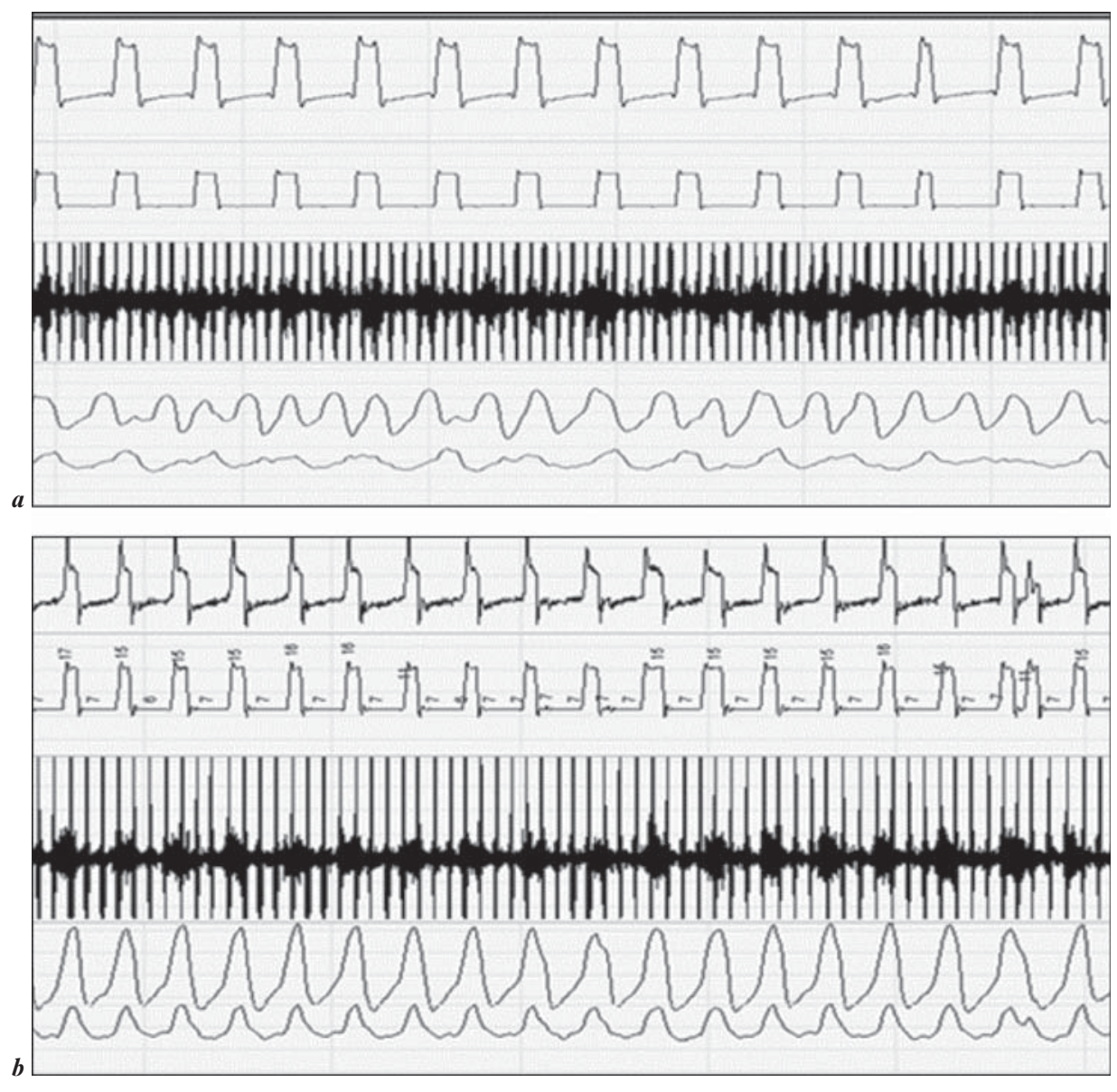

Fig. 1. (for Abstract 23)

Panel A: Example of unrewarded inspiratory efforts with initial ventilator settings.

From top to bottom: flow, pressure, EMG of the diaphragm, thoracic and abdominal belts. This 60 second epoch shows major patient-ventilator asynchrony. Unrewarded efforts can easily be identified by comparing the EMG trace or that of the abdominal and thoracic belts with the flow or pressure traces. The patients inspiratory efforts do not trigger the ventilator which is on its back-up rate.

Panel B: Correction of patient ventilator asynchrony by adjustment of ventilator sattings with polysomnography. Flow, pressure, right diaphragm EMG and abdominal and thoracic belts are perfectly synchorinized. All inspiration efforts trigger the ventilator resulting in an increased respiratory rate.

Fig. 2. (for Abstract 23)

Correction of 'de-ventilation' dyspnea by adjusting ventilator settings with polysomnography is illustrated for each individual patient on panel a. On a $0-10$ visual analog scale, subjective assessment of ventilator synchronization (Panel b), leaks (Panel c) and overall quality of sleep (Panel d) are all improved.

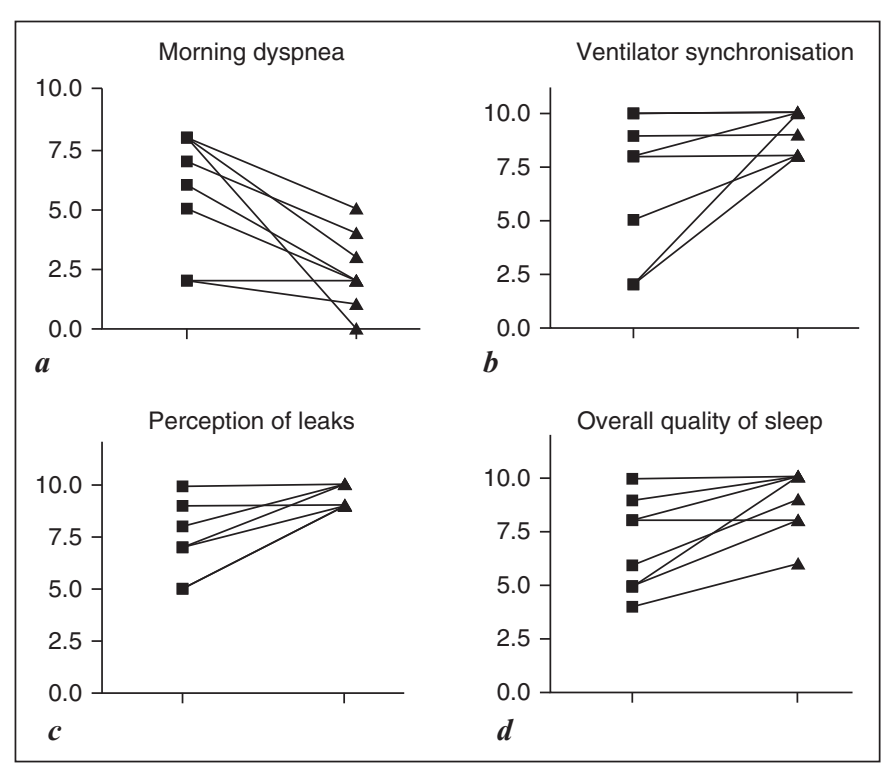


etry. Subjects with known asthma were excluded from the study. All patients according to the GOLD guidelines were treated with tiotropium. After 4 weeks of tiotropium only patients were randomized in a double blind manner to either additionally budesonid twice daily (total daily dose of $1600 \mathrm{mcg}$ ) or placebo for another 3 months. At all visits lung function, AHR to mannitol, Quality of Life (SGRQ), Symptom Score (MRC Dyspnoe Scale) and exhaled nitric oxide levels were assessed. AHR was defined as a PD 15 of $635 \mathrm{mg}$ or less. Response-dose-ratio (RDR) was calculated as the percent fall in FEV1 at the last dose divided by totally dose of mannitol administered.

Results: At baseline AHR to mannitol challenge was found in 38 patients (56\%). After 3 month of treatment with ICS there were no significant changes from baseline regarding FEV1\% pred. (2.46 CI $[-1.01 ; 5.93] \mathrm{p}=0.162)$, MRC Dyspnoe Scale $(0.4$ CI $[0.1 ; 1.7] \mathrm{p}=0.22)$ and Quality of Life (SGRQ) (-3.21 CI $[-8.47 ; 2.05] \mathrm{p}=0.227)$ as compared to placebo. Statistically we found slightly lower values of exhaled nitric oxide $(50 \mathrm{ml} / \mathrm{s})$ $(-5.48 \mathrm{CI}[-11.16 ; 0.19] \mathrm{p}=0.058)$ and a decline in $\log 10 \mathrm{RDR}$ (response dose ratio) $(-0.26 \mathrm{CI}[-0.49 ;-0.03] \mathrm{p}=0.025)$ after ICS treatment. However improvement in SGRQ after ICS treatment was significant better in subjects presenting with AHR to mannitol $(-8.64$ CI $[-16.11 ;-1.17] \mathrm{p}=0.024)$. AHR to mannitol correlated with an improvement of FEV $1 \%$ pred. of about $5 \%$ as compared to placebo $(p=0.09)$, but this effect could not be discriminated from subjects with negative mannitol challenge $(0.03$ CI $[-5.02 ; 5.09](\mathrm{p}=0.989)$. Exhaled nitric oxide did not change over time in dependence of AHR (0.23 CI [-8.49; 8.95] $\mathrm{p}=0.958$ ). Our findings were not influenced by current smoking status.

Conclusion: Our observations suggest that AHR improves and seems to result in a better quality of life after ICS treatment in mild to moderate COPD.

\section{5 \\ Is the Severity of Airflow Limitation Associated with Vascular Function in COPD?}

C.F. Clarenbach, A.-C. Stöwhas, G. Mettler, A.J.R. van Gestel, E.W. Russi, M. Kohler

University Hospital of Zurich, Zurich

Background: Cardiovascular disease plays an important role regarding the morbidity and mortality in patients with COPD. Preliminary data suggest that impaired endothelial function and increased arterial stiffness may be mechanisms underpinning the association between COPD and cardiovascular disease. Endothelial function and arterial stiffness can be assessed by flow-mediated dilatation (FMD) of the brachial artery and by radial applanation tonometry derived augmentation index (AIx), respectively. Our objective was to evaluate whether there is an association between the severity of airflow limitation and vascular function.

Methods: In 75 patients ( 45 males) with a mean age (SD) of 62.4 (7.3) years and confirmed COPD according to GOLDguidelines, lung function (FEV1), endothelial function (FMD) and arterial stiffness (AIx) were measured.

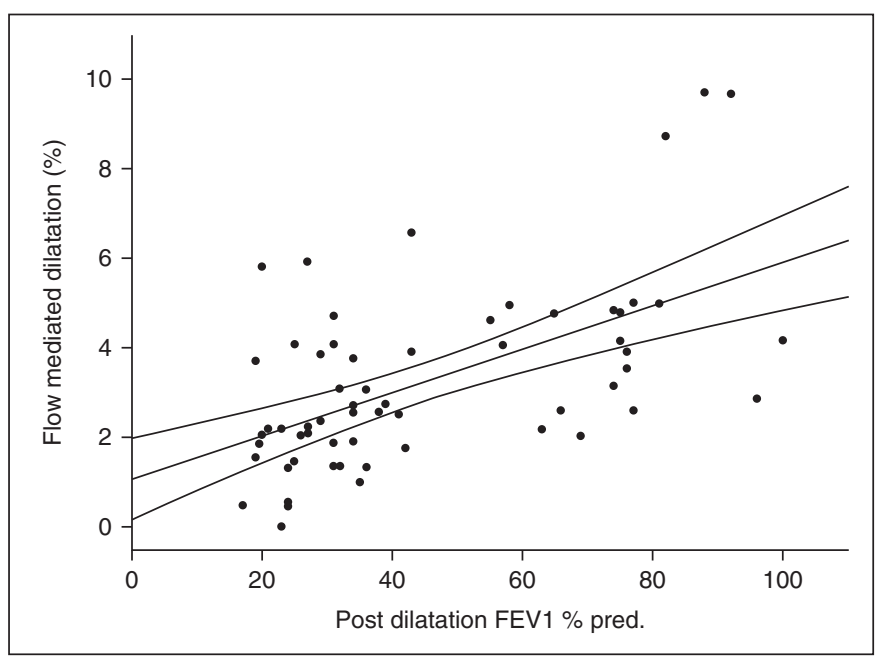

Fig. 1.

Results: Endothelial function as assessed by FMD was strongly correlated with the severity of airflow limitation $(r=0.57, p<0.001$, figure 1) and this association remained significant after correction for smoking history, age, blood pressure and cholesterol level. Mean FMD (SD) was nearly normal in patients with GOLD stage I: 6.4 (3.4)\% but was progressively impaired in patients with GOLD stage II: 4.2 (1.7)\%, III: 2.9 (1.4)\% and stage IV: $2.2(0.3) \%$ ( $\mathrm{p}<0.001$ for comparison between groups). Arterial stiffness as assessed by AIx was not associated with the severity of airflow limitation $(\mathrm{r}=-0.13, \mathrm{p}=0.31)$.

Conclusions: COPD seems to be associated with impaired endothelial function but not with increased arterial stiffness. Therefore endothelial dysfunction may play a role in the pathogenesis of vascular disease in patients with COPD.

\section{6 \\ COPD is Associated with Increased Sympathetic Nervous System Activation}

\section{C.F. Clarenbach, A.-C. Stöwhas, G. Mettler, A.J.R. van Gestel, O. Senn, E.W. Russi, M. Kohler}

University Hospital of Zurich, Zurich

Background: Cardiovascular disease plays an important role regarding the morbidity and mortality in patients with COPD. Up to $50 \%$ of all deaths in patients with COPD are caused by cardiovascular disease. Mechanisms underpinning the association between cardiovascular disease and COPD are largely unknown. Preliminary data suggest that COPD might lead to increased sympathetic activity. Thus, our aim was to test the hypothesis that the severity of airflow limitation is associated with augmented sympathetic activity. 


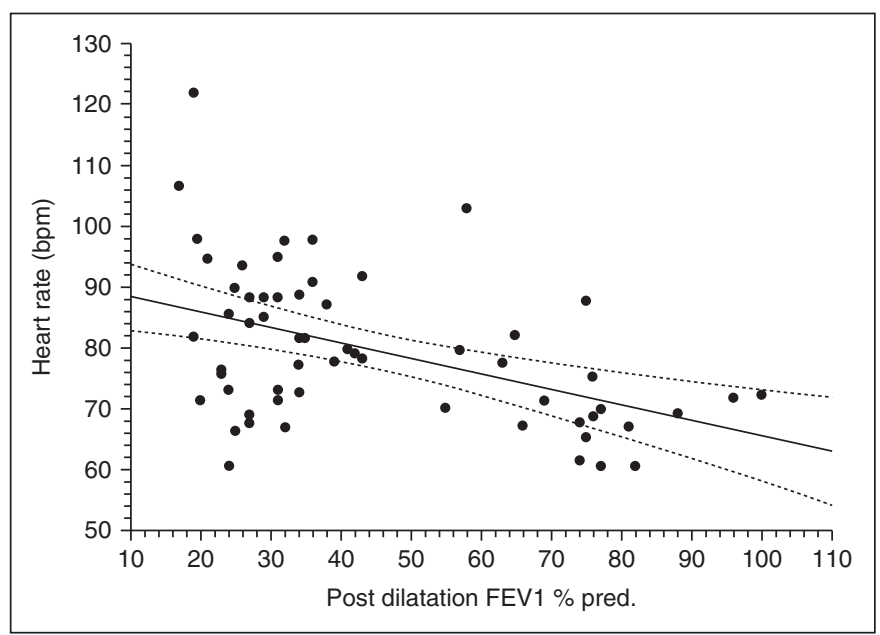

Fig. 1.

Methods: In 63 patients (39 males) with a mean age (SD) of 62.2 (7.7) years and confirmed COPD according to GOLDguidelines, airflow limitation (FEV1 pred.) was measured by spirometry and blood pressure, resting heart rate, heart rate variability as well as baroreflex sensitivity were assessed by non-invasive beat-to beat analysis of pulse pressure (Finapres ${ }^{\circledR}$ ) during 5 minutes.

Results: $5 \%$ of the examined patients were classified as GOLD stage I, $29 \%$ as GOLD stage II, $31 \%$ as GOLD stage III and $35 \%$ as GOLD stage IV.

In univariate analysis the severity of airflow limitation (FEV1 pred.) was correlated with resting heart rate $(\mathrm{r}=-0.47, \mathrm{p}<0.01$, figure 1$)$ and baroreflex sensitivity $(\mathrm{r}=0.33, \mathrm{p}<0.01)$. There was a trend towards a lower heart rate variability in patients with more severe airflow limitation $(\mathrm{r}=0.22, \mathrm{p}=0.09)$. The severity of airflow limitation was not correlated with blood pressure.

In multivariate analysis the severity of airflow limitation (FEV1 pred.) remained significantly associated with heart rate and baroreflex sensitivity after correction for smoking status as well as pack years, body-mass-index, blood pressure, and the use of beta blockers.

Conclusions: There is an association between the severity of airflow limitation and sympathetic nervous system activity in patients with COPD. Therefore increased sympathetic nervous system activity may be a mechanism linking COPD with cardiovascular disease.

\section{7}

\section{Oxygen Kinetics During 6-minute Walk Tests Using} Mobile Telemetric Cardiopulmonary Monitoring

S. Condrau' , L. Kern'², F. Baty², J. Wiegand ${ }^{3}$, A. Azzola ${ }^{4}$, M. Tamm ${ }^{5}$, M.H. Brutsche ${ }^{2}$

${ }^{1}$ Hospital of Interlaken, Interlaken, ${ }^{2}$ Cantonal Hospital St. Gallen, St. Gallen, ${ }^{3}$ Inselspital, Bern, ${ }^{4}$ Ospedale Regionale Lugano, Lugano, ${ }^{5}$ University Hospital of Basel, Basel

Background: The mean response time (MRT) assessed by cardiopulmonary exercise testing (CPET) is a prognostic factor for hospitalization, mortality and transplantation in patients with congestive heart failure (CHF). It is not known, whether MRT can also be calculated using Mobile Telemetric Cardiopulmonary Monitoring (MOB) during a conventional non-encouraged 6-minute walk test (6MWT). MOB enhanced 6MWT has been shown to be a valuable tool to identify factors limiting exercise in patients irrespective of their underlying disease.

Rationale: The aim of the study was to assess MRT in a MOB setting and to test its predictive value in several disease categories.

Material and Methods: Oxygen consumption profiles of 203 patients and 16 healthy controls were obtained using $\mathrm{MOB}$ $\left(\right.$ Oxycon Mobile ${ }^{\circledast}$ ) during a 6MWT. Patient characteristics are depicted in Table 1. Curves were fitted using asymptotic regression models and the following parameters were assessed: $\mathrm{O} 2$-deficit, baseline and peak $\mathrm{O} 2$-consumption during exercise and mean response time (MRT). In contrast to CPET, MOB does not provide information on work load. Therefore, MRT was reparametrized $\left(\mathrm{MRT}^{*}=\mathrm{MRT} / \mathrm{dVO} 2\right)$ to correct for this variable. The differences in $\mathrm{MRT}^{\star}$ between disease categories were tested using linear models (ANOVA F-test). The association between $\mathrm{MRT}^{\star}$ and patient outcome - time to hospitalization / death - was tested using Cox proportional hazards regression.

Results: Unadjusted MRT assessed by MOB did not differ between patients and healthy controls. O2-deficit and $\mathrm{MRT}^{\star}$ were significantly increased in patients compared to healthy controls $(\mathrm{p}<0.001)$. There was no significant difference between different disease categories. MRT $^{\star}$ significantly predicted the patient outcome only in patients with CHF ( $\mathrm{p}=0.004$, Figure 1$)$.

Conclusion: Compared to healthy controls $\mathrm{MRT}^{\star}$ was generally increased in all patient groups. $\mathrm{MRT}^{\star}$ is a significant prognostic factor for patients with CHF but not for patients suffering from chronic lung disease. We could therefore reproduce the results of previous results obtained with $\mathrm{CPET}$ using $\mathrm{MOB}$ during a 6MWT.

Table 1. Patient characteristics (mean $+/-$ SD) (for Abstract 27)

\begin{tabular}{|c|c|c|c|c|c|}
\hline & Healthy & Restrictive & COPD & PAHT & $\mathrm{CHF}$ \\
\hline Sex (females) & 8 & 21 & 39 & 36 & 12 \\
\hline BMI & $24+/-4$ & $30+/-7$ & $26+/-6$ & $27+/-6$ & $30+/-8$ \\
\hline Walking distance, $\mathrm{m}$ & $720+/-83$ & $381+/-110$ & $357+/-110$ & $338+/-120$ & $340+/-91$ \\
\hline
\end{tabular}




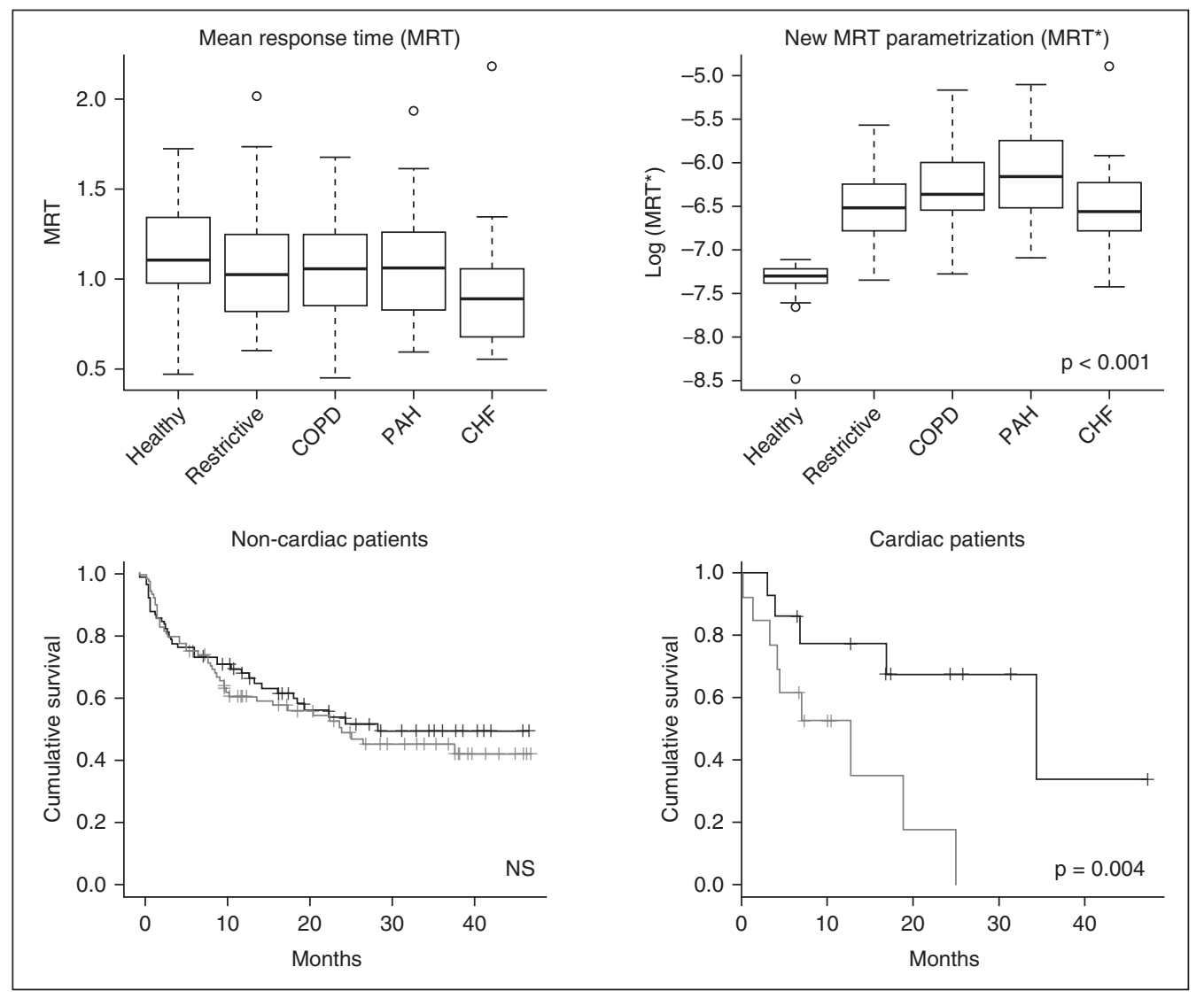

Fig. 1. (for Abstract 27)

\section{8 \\ Nasal Nitrite Oxide Measurement in Preschool Children: Feasibility and Validation of a Tidal Breathing Technique with a Straw}

\section{A. Jung ${ }^{1}$, C. Geidel ${ }^{1}$, I. Heinrichs ${ }^{1}$, A. Möller ${ }^{2}$, G. Menz ${ }^{1}$, R. Lauener ${ }^{1}$ \\ ${ }^{1}$ Hochgebirgsklinik Davos, Davos, ${ }^{2}$ University Children's Hospital, Zurich}

Background: Screening for primary ciliary dyskinesia (PCD) in preschool children is difficult, as non-invasive techniques such as measurement of nasal nitrite oxide (nNO) have not been standardized and reference values are lacking. This study aimed to compare a conventional nNO measurement technique with a tidal breathing technique through a medium-sized straw.

Methods: nNO was assessed by the Niox Flex system in children and adults with chronic rhino-sinusitis (CRS), confirmed PCD and in healthy controls. Measurements consisted of 2 tests with subjects holding their breath ( $\mathrm{BH}$ technique), followed by 2 tests with tidal breathing through a straw (5 mm diameter, 210 mm length; TB technique). In both settings, a test was considered valid when $\mathrm{NO}$ concentration reached a stable plateau.

Results: 88 subjects (median age 9.8 yrs) were recruited. 71 $(81 \%)$ or $77(88 \%)$ subjects performed either a valid $\mathrm{BH}$ or a valid TB test, respectively. In the preschool age group (age $<6$ yrs; $n=22), 19$ subjects $(86 \%)$ had a valid TB test with only 10 individuals (45\%) performing a valid $\mathrm{BH}$ test.

Median nNO levels were higher using the TB technique as compared to the $\mathrm{BH}$ technique in healthy controls and subjects with CRS $(p<0.001$ and $p=0.009$, respectively). For the PCD group, results didn't differ between both techniques. A high correlation between $\mathrm{TB}$ and $\mathrm{BD}$ values were observed $(\mathrm{r} 2=0.954$, $\mathrm{p}<0.001$ ).

Median nNO values were significantly lower for the CRS group compared to the control group $(\mathrm{p}<0.001)$. Ranges of NO values greatly overlapped between controls and CRS and had therefore no predictive power, whereas the method discriminated well between individuals with PCD and subjects without PCD.

For the control group, level of nNO values were independent of age $\left(r^{2}=0.044\right.$, n.s. $)$. When controls were stratified for age $(<6$ yrs $n=9,6-15$ yrs $n=18,>=16$ yrs $n=21)$, there was a trend towards lower NO levels for younger children in the preschool age group $\left(\mathrm{r}^{2}=0.341, \mathrm{p}<0.1\right)$.

Conclusion: Nasal NO measurement by TB with a straw is a feasible and valid method in all age groups. Results correlate well with $\mathrm{nNO}$ levels measured by conventional techniques. Almost all preschool children were able to perform a valid TB test. The current findings point to lower nasal NO levels in the very young. A larger study is currently on the way to determine reference values in preschool children on the most widespread devices, using the established TB method with a straw. 


\section{Free Communications 2 Basic Research}

\section{9 \\ Deficient Production of Interferon Stimulated Genes Upon Rhinovirus Infection in Cystic Fibrosis Airway Epithelial Cells}

\author{
E. Kieninger', M. Vareille', M. Alves' ${ }^{1}$, B.S. Kopf', T. Geiser ${ }^{3}$, \\ S.L. Johnston ${ }^{4}$, M.R. Edwards ${ }^{4}$, N. Regamey ${ }^{1}$ \\ ${ }^{1}$ University Children's Hospital Berne, Berne, ${ }^{2}$ University of \\ Berne, Berne, ${ }^{3}$ University Hospital of Berne, Berne, ${ }^{4}$ Imperial \\ College London, London, UK
}

Background: Virus infections play an important role in cystic fibrosis (CF) respiratory morbidity but their contribution to lung disease progression is poorly understood. We recently described deficient innate immune responses to rhinovirus (RV) infection in CF airway epithelial cells, characterized by impaired type I and III interferon (IFN-beta and -lambda) production, resulting in increased virus replication. To further study downstream effects of impaired IFN induction in CF cells, we now investigated the expression of IFN-stimulated genes (ISGs), which are important for the production of several anti-viral proteins.

Methods: Epithelial CF and non-CF cell lines (UNCCF2T/ UNCN2T, CFBE41o-/16HBE14o-) were used in in vitro studies. Epithelial cells were cultured and infected with rhinoviruses (RV$16, \mathrm{RV}-1 \mathrm{~B}$ ) at a multiplicity of infection of 2. Quantitative PCR was carried out to assess the induction of ISGs. Exogenous IFNbeta and -lambda $(100 \mathrm{pg} / \mathrm{ml})$ was added before and after virus infection.

Results: Expression of all ISGs was induced in both CF and control cells upon virus infection but CF cells expressed 100 to 1000 times less ISGs including MxA, 2',5'-OAS, viperin and NOS than control cells (all $\mathrm{p}<0.05$ ). ISG expression and RV replication were inversely related (e.g. MxA: $r=-0.79, p=0.001$ ), and there was a positive correlation between ISG expression and IFN-beta production (e.g. 2',5'-OAS: $\mathrm{r}=0.74, \mathrm{p}=0.004$ ), and between ISG expression and IFN-lambda production (e.g. NOS2: $\mathrm{r}=0.65$, $\mathrm{p}=0.01)$. Both exogenous IFN-beta and -lambda $(100 \mathrm{pg} / \mathrm{ml})$ increased levels of ISGs to the level of control cells, with a more pronounced effect of IFN-beta.

Conclusion: Induction of ISGs upon RV infection is defective in $\mathrm{CF}$, indicating a profound impairment of the anti-viral early innate response to virus infection. Addition of exogenous IFNs, in particular of IFN-beta, restores anti-viral pathways and virus control in CF cells. These mechanisms may contribute to the increased respiratory morbidity after virus infection in $\mathrm{CF}$ and suggest the potential use of IFNs in the treatment of virus-induced CF exacerbations.

\section{0}

\section{Lung on Chip: In vitro HGF Effects on Injured Lung} Epithelial Cells in a Microfluidic System

\author{
P. Sallin 1', L. Barbe ${ }^{2}$, A. Gazdhar' ${ }^{1}$ T. Geiser ${ }^{3}$, O. Guenat ${ }^{1}$ \\ ${ }^{1}$ University of Berne, Berne, ${ }^{2}$ CSEM, Landquart, ${ }^{3}$ University \\ Hospital of Berne, Berne
}

Background: Microfluidic systems have become competitive tools in the in-vitro modelling of diseases and promising alternatives to animal studies. They allow, in particular, obtaining more in-vivo like conditions for cellular assays. Research in idiopathic pulmonary fibrosis could benefit from this novel methodological approach to understand the pathophysiology of the disease and develop efficient therapies. The use of hepatocyte growth factor (HGF) for alveolar reepithelisation is one main focus of the current research. In this study, we sought to develop a new microfluidic system to analyze the effects of HGF on injured alveolar epithelial cells.

Methods: Microfluidic systems in polydimethylsiloxane were fabricated by soft lithography. The human alveolar A549 epithelial cells were seeded and studied in these microfluidic systems with media perfusion $(1 \mu \mathrm{l} / 30$ minutes). Injury tests were made on the cells by the perfusion with media containing $\mathrm{H}_{2} \mathrm{O}_{2}(0.0625 \mu \mathrm{M}$, $0.625 \mu \mathrm{M}, 1.25 \mu \mathrm{M})$ or bleomycin $(0.002 \mathrm{U} / \mathrm{ml}, 0.02 \mathrm{U} / \mathrm{ml}, 0.2 \mathrm{U} / \mathrm{ml})$ during 6 or $18 \mathrm{~h}$. The degree of injury was then assessed by a metabolic and an apoptotic assays. Wound assays were performed with a central laminar flow of $10 x$ concentrated trypsin/EDTA. Monitoring of wound closure with media \pm HGF $(10 \mathrm{ng} / \mathrm{ml}) \pm 10 \%$ FBS and \pm pre-incubation of the cells with mitomycin C $(10 \mu \mathrm{g} / \mathrm{ml})$ was assessed by time lapse microscopy. The degree of wound closure was calculated as a percentage of initial wound area.

Results: The alveolar A549 epithelial cells grew and proliferated in the microfluidic system. The injury tests permitted to choose the most adapted concentrations of bleomycin and $\mathrm{H}_{2} \mathrm{O}_{2}$ for further experiments with HGF. In the wound closure assay with $10 \%$ FBS, the degree of wound closure after 5 hours was $53.3 \pm 1.3 \%$ with HGF compared to $9.8 \pm 2.4 \%$ without HGF $(\mathrm{p}<0.001)$. In the microsystems without FBS, this degree after 5 hours was $47.3 \pm 28 \%$ with HGF compared to $14.1 \pm 11.7 \%$ without HGF $(p<0.05)$. In the epithelial wound closure assay with preincubation of the cells with mitomycin $\mathrm{C}$, no difference was observed at all time points between the microsystems with or without HGF.

Conclusion: We present a novel microfluidic model that allows culture, injury and wounding of A549 epithelial cells and represents the first step towards the development of an in-vitro reconstitution of the alveolar-capillary interface. We were also able to confirm that HGF increased alveolar epithelial repair in vitro in this microfluidic system. 


\section{1 \\ Epithelial-cell Conditioned Media Strongly Modulates the Function and Differentiation of Monocytes and Dendritic Cells Towards Regulatory Cells \\ V. Cesson ${ }^{1}$, K. van der Sluijs' ${ }^{2}$, L.P. Nicod ${ }^{1}$, C. Obregon ${ }^{1}$ \\ ${ }^{1}$ CHUV, Lausanne, ${ }^{2}$ Service of Pneumology, Amsterdam, NL}

Lung DCs, due to their localization, must be profoundly influenced by the epithelial milieu. Thus, we aimed to study the role of lung epithelial environment, in the function of monocytes (Mo) and their differentiation to DCs.

Monocytes (Mo) were cultured with epithelial supernatant (E-SN) both from A-549 or B2B epithelial cells stimulated with IL-1beta or respiratory syncytial virus (RSV) and compared to Mo cultivated in Mock conditions or culture with supplemental factors IL-4 /GM-CSF. Maturation markers, DC myeloid or plasmacytoid subset and monocyte cell markers were analysed. DC functionality was examined by the release of cytokines as well as the capacity to present allo antigens or induce T regs.

The epithelial supernatant (ESN) stimulated or mock conditions induced a differentiation of Mo into monocyte-DC like as observed by the upregulation of CD86, mannose receptor but maintained expression of CD14 and CD163. Surprisingly, E-SN stimulated with IL-1beta or RSV induced a transient expression of BDCA-2, and increased expression of BDCA-4 and CD123 compared to mock conditions.

Functionally, Mo cultured in the E-SN maintain an immature phenotype after stimulation with LPS, and poly I:C maintaining a low expression of CD80, CD83 and no expression of CD86 and CCR7. Furthermore, the E-SN has kept an important immunosuppression inhibiting the release of IL-12 and TNF-a, but do not inhibit the release of IL-6, IL-10. In addition, they maintain the release of IFN-alpha and IFN-lambda upon TLR-9 stimulation. Functionally, cells cultured in ESN failed to present allo antigens and interestingly they have an increased capacity to expand Tregs.

To analyse if the E-SN modulation was reversible, cells were cultured in the ESN then washed and cultured with GM-CSF/IL4 or viceversa. Interestingly, ESN inhibit not only the expression of maturation markers after LPS stimulation in Mo but also inhibit the expression in already differentiated DC.

Lung epithelial supernatants were shown to induce plasmacytoid features in DCs, with an important regulatory function. The epithelium seem to maintain the cells in their environment avoiding migration of DCs to the lymph-nodes, driving the local immunity towards a Th2 by the release of IL-10 and shutting down the release of IL-12. Understanding DCs plasticity may be important for the control of $\mathrm{T}$ cell polarization towards immune response or tolerance.

\section{2 \\ Particle Size Determines Uptake Efficiency of Different Antigen Presenting Cell Populations in the Respiratory Tract During Steady State and Allergic Airways Inflammation}

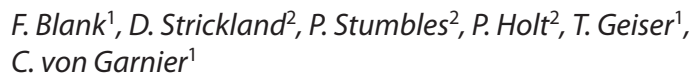

${ }^{1}$ Berne University Hospital, Berne, ${ }^{2}$ Telethon Institute for Child Health Research, Perth, AUS

Increasing research is being conducted to develop novel biomedical particles for diagnostic and therapeutic applications. Effects of biomedical particles on lung antigen presenting cells (APC) like lung parenchymal dendritic cells (LPDC) and alveolar macrophages (AM), immunological key players that underlie stringent control mechanisms to maintain immunological homeostasis, are poorly characterised to date.

To study the effect of particle size on the uptake efficiency of lung APC during steady state and allergic airways disease (ovalbumin sensitized and challenged), BALB/c mice were intra-nasally instilled with different sized fluorescent polystyrene particles $(50 \mathrm{~nm}, 100 \mathrm{~nm}, 200 \mathrm{~nm}, 1000 \mathrm{~nm}$ in diameter). Two h after instillation bronchoalveolar lavage fluid, and peripheral lung tissue were collected and single cell suspensions were obtained. Cell phenotypes and particle uptake were analysed using flow cytometry.

Different lung APC populations could be characterized and monitored during particle exposure in naive and allergic mice. Independent of size most of the particles were found in AM in naive and allergic mice. About 20\% less AM were involved in uptake of $50 \mathrm{~nm}$ particles compared to bigger particles in naive but not in allergic mice. More LPDC were involved in uptake of $50 \mathrm{~nm}$ particles compared to bigger particles in naive and allergic mice.

Our study shows that size needs to be thoroughly addressed when designing appropriate drug or vaccine carries for interaction with the cellular lung immune system.

33

\section{SERPINA1 MS and MZ Heterozygotes and Lung Function Decline in the SAPALDIA Cohort Study}

G.A. Thun ${ }^{1}$, I. Ferrarotti ${ }^{2}$, M. Imboden ${ }^{1}$, T. Rochat ${ }^{3}$, M. Zorzetto ${ }^{2}$, S. Ottaviani ${ }^{2}$, E.W. Russi ${ }^{4}$, M. Luisetti ${ }^{2}$, N.M. Probst-Hensch ${ }^{1}$

${ }^{1}$ Swiss TPH / University of Basel, Basel; ${ }^{2}$ University of Pavia, Pavia, I, ${ }^{3}$ University Hospital of Geneva, Geneva, ${ }^{4}$ University Hospital of Zurich, Zurich

The only ascertained genetic risk factor for chronic obstructive pulmonary disease (COPD) is the concentration of alpha1-antitrypsin in the blood, which is dictated by SERPINA1 genotypes in a co-dominant fashion. Severe alpha1-antitrypsin deficiency has a well established adverse impact on the longitudinal course of respiratory health, but the effect of gene variants resulting in intermediate alpha1-antitrypsin deficiency (most frequently SERPINA1 MS and MZ) is far less clear. Therefore, we tested a possible unfa- 
vourable effect of SERPINA1 MS and MZ genotypes on the change in lung function in different subgroups of the large, populationbased cohort study SAPALDIA.

The study population represents the three major Swiss language regions and includes more than 5000 subjects from whom SERPINA1 genotypes for $\mathrm{S}$ and $\mathrm{Z}$ alleles, alpha1-antitrypsin blood levels, and spirometry at baseline and after 11 years follow up are available. All analyses were performed by applying multiple regression models which were adjusted for gender, age, recruiting region, smoking status, baseline BMI and BMI change between baseline and follow up.

While we did not find an overall significant effect of MS or MZ compared to the wildtype MM carriers on lung function decline, $\mathrm{MZ}$ seems to be a risk factor for obese patients (annual decline in the forced mid expiratory flow (FEF 25-75\%) $56.4 \mathrm{ml}(\mathrm{MM})$ vs. $92.6 \mathrm{ml}(\mathrm{MZ}), \mathrm{p}=0.02)$ and smokers $(72.7 \mathrm{ml}(\mathrm{MM})$ vs. $89.3 \mathrm{ml}$ $(\mathrm{MZ}), \mathrm{p}=0.04)$. Moreover, smoking history seems to influence the accelerated decline in $\mathrm{MZ}$ individuals in a dose-responsive and gender dependent manner. Equivalent, but less strong associations were observed for the decline in the FEV1/FVC ratio.

We suggest that the presence of one $\mathrm{Z}$ allele, which comprises between 2 and $3 \%$ of the Swiss population, may already be sufficient to accelerate lung function decline in subgroups exposed to high oxidative stress.

\section{4 \\ Confounding Factors Influencing Accuracy of DLCO- Measurements: Comparisons of Two Different Devices \\ R. Kraemer ${ }^{1}$, C. Buess ${ }^{2}$, P. Ballinary ${ }^{3}$, J. Barandun ${ }^{1}$, T. Scherer $^{1}$, A. Piecyk ${ }^{1}$, K. Klingler ${ }^{1}$ \\ ${ }^{1}$ LungenZentrum Zürich, Zürich, ${ }^{2}$ ndd Medical Technologies, Zürich, ${ }^{3}$ Institut für Psychologie, Bern}

Rationale: Inter- and intra-laboratory variability and equipment verification for measuring diffusion capacity of the lung for carbon monoxide (DLCO) have educated some disturbing concern recently $[1,2]$. To detect small changes in DLCO, accurate measurements of volumes along the test procedure of DLCO measurements have to be faced.

Objectives: (1) to assess the accuracy of DLCO measurements comparing the two devices EasyOnePro (EOP) and ZAN300 (ZAN) by evaluating the variability of measurements of static lung volumes. (2) to determine the discrimination of the two devices between healthy subjects, asthmatic and COPD patients.

Methods: Lung function testing including spirometry, wholebody plethysmography and DLCO measurements were performed in 36 subjects ( 11 healthy, 13 asthmatics, 12 COPD adults), assessing device accuracy with respect to measurements of residual volume (RV), total lung capacity (TLC), alveolar volume (VA) and hence the calculation of TLco and Kco.

Results: Significant differences regarding measurements of lung volumes were discovered. EOP measured lower RV $(p=0.004)$, higher TLC ( $\mathrm{p}=0.001)$, lower RV/TLC ratios $(\mathrm{p}<0.001)$ and hence higher VA $(p=0.003)$. Therefore, by inclusion of RV/TLC into the regression model, substantially better discrimination was achieved.
Although with both devices using TLco-FEV1/FRC plots distinction between healthy subjects, asthmatic and COPD patients could be achieved, EOP presented with better Wilks'Lambda factors of discrimination ( $\mathrm{chi}^{2}$ for TLco EOP: 46.9; $\mathrm{p}<0.001$, versus ZAN: 37.3; $\mathrm{p}<0.001)$.

Conclusions: Precise measurement of lung volumes along with DLCO-measurements seems to be essential in order to access accurate VA. Especially in patients with severe lung involvement minimal discrepancies regarding RV/TLC, and hence VA influence the DLCO-calculation. It is suggested that a part from potentially different guidance of the patient by the technician performing the tests, major factors are measurements of lung volumes. Flow measurements with EOP are performed by ultrasonic technology (no resistance from the apparatus) presumed to be superior to the pneumotachographic technic of ZAN with an instrumental resistance of $0.03 \mathrm{kPa} / \mathrm{l} / \mathrm{s}$.

[1] Eur Respir J. 2009, 33:828-834.

[2] Eur Respir J. 2009 Aug;34:523-4.

\section{Free Communications 3 Clinical Research: Sleep}

\author{
58 \\ CPAP Improves Endothelial Function in Minimally \\ Symptomatic OSA Patients: Results from the MOSAIC \\ Trial \\ M. Kohler', S. Craig', J.C.T. Pepperell ${ }^{3}$, D. Nicoll ${ }^{2}$, D. Bratton ${ }^{4}$, \\ A. Nunn ${ }^{4}$, P. Leeson ${ }^{2}$, R.J.O. Davies ${ }^{2}$, J.R. Stradling ${ }^{2}$ \\ ${ }^{1}$ University Hopsital Zurich, Zurich, ${ }^{2}$ Oxford Centre for \\ Respiratory Medicine, Oxford, UK, ${ }^{3}$ Somerset Centre for \\ Respiratory Medicine, Taunton, UK, ${ }^{4}$ Clinical Trials Unit MRC, \\ London, UK
}

Background: CPAP treatment for symptomatic OSA improves measures of cardiovascular risk, such as endothelial function and arterial stiffness. Minimally symptomatic OSA is far more prevalent than symptomatic OSA but the effects of CPAP on endothelial function and arterial stiffness in these patients are unknown.

Methods: 245 patients with minimally symptomatic OSA were randomised to 6 months of CPAP or standard care. 208 patients attended their follow-up visit within the predefined time window and had complete measurements of arterial stiffness (augmentation index, AIx) and 64 patients had endothelial function measurements by brachial artery flow-mediated dilatation (FMD). Multivariable analyses adjusting for baseline measurements and minimisation factors were performed to assess the effect of CPAP treatment on FMD and AIx compared to standard care.

Results: The mean \pm SD baseline oxygen desaturation index and Epworth sleepiness score (ESS) of the 208 patients (age $58 \pm 7.3$ years, 31 (14.9\%) females) were $13.7 \pm 12.8$ and $8.3 \pm 4.2$, respectively. There was no effect of CPAP on arterial stiffness (AIx, $-1.4 \%, 95 \%$ CI -3.6 to $0.9 \%, p=0.23)$, but CPAP improved 
endothelial function (FMD, $+2.1 \%$, 95\% CI 1.0 to $3.2 \%$, p < 0.0001 ). CPAP improved daytime sleepiness as assessed by ESS $(-2.1,95 \%$ CI -2.9 to $-1.4, \mathrm{p}<0.0001)$. A one hour increase in CPAP usage was estimated to improve FMD by $0.5 \%$ (95\% CI 0.0 to $0.9 \%$, $\mathrm{p}=0.04)$, but there was no statistically significant effect of CPAP usage on AIx.

Conclusions: CPAP improves endothelial function, but not arterial stiffness in minimally symptomatic OSA. Thus CPAP may reduce cardiovascular risk in patients with minimally symptomatic OSA.

\section{9}

AutoCPAP Devices Accurately Identify Obstructive Sleep Apnoea Patients with Residual Apnoea During Treatment

L. Hess' ${ }^{1}$, T.D. Latshang ${ }^{1}$, C.M. Lo Cascio ${ }^{1}$, M. Kohler ${ }^{1}$, R. Thurnheer ${ }^{2}$, K.E. Bloch ${ }^{1}$

${ }^{1}$ University Hospital of Zurich, Zurich, ${ }^{2}$ Kantonsspital

Münsterlingen, Münsterlingen

Background: In the long-term care of patients with the obstructive sleep apnea syndrome (OSA) objective data on the effectiveness of CPAP treatment would be desirable. Although CPAP devices record the apnea/hypopnea index (AHI) during use the accuracy and clinical relevance of these measurements are not known. Therefore, we compared AHI recorded by CPAP devices with corresponding values measured during in-laboratory polygraphic sleep studies. The hypothesis was that the AHI derived from autoCPAP devices accurately predict an elevated AHI during a sleep study.

Methods: 48 OSA patients, mean age \pm SD $54 \pm 6 y$, baseline AHI $52.5 \pm 21.6 / \mathrm{h}$, underwent polygraphic sleep studies once or twice within a few months during long-term autoCPAP therapy (ResMed S8, Philips Respironics REMstar). The memory of CPAP devices was downloaded into a PC for the night of the sleep study as well as for the 7 nights and the 3 months preceding the study. AHI derived from CPAP devices and sleep studies were analyzed.

Results: A total of 60 sleep studies and corresponding CPAP downloads were available. Mean \pm SD machine use was $6: 44 \pm 1: 25 \mathrm{~h}$, the allowed pressure range was $5-15 \mathrm{~cm} \mathrm{H} 2 \mathrm{O}$, mean applied CPAP was $10.9 \pm 1.8 \mathrm{~cm} \mathrm{H} 2 \mathrm{O}$. The AHI derived from polygraphic sleep studies was $5.0 \pm 6.2 / \mathrm{h}$, the mean difference (bias) of the corresponding AHI from CPAP devices was $1.8 / \mathrm{h}$, $95 \%$ confidence interval -7.4 to $+11.0 / \mathrm{h}$. The mean difference between the maximal and minimal CPAP derived AHI in the week preceding the sleep study was $6.1 \pm 5.1 / \mathrm{h}$. In 22 out of 60 polygraphic sleep studies, the AHI was elevated $>5 / \mathrm{h}$. The accuracy of the AHI from CPAP devices to correctly predict abnormal sleep studies with $\mathrm{AHI}>5 / \mathrm{h}$ was quantified by the area under the receiver operating characteristic curve of 0.87 (95\% CI 0.78 to 0.96). The sensitivity and specificity of an AHI $\geq 5 / \mathrm{h}$ from CPAP devices to identify a polygraphic AHI $>5 /$ h were $86 \%$ and $75 \%$, respectively; the negative and positive predictive values were $93 \%$ and $56 \%$, respectively.
Conclusions: AutoCPAP devices accurately identify OSA patients with elevated AHI during long-term treatment of OSA. Since the night-to-night variability of the AHI is high, AHI derived from autoCPAP devices over multiple nights in the home setting might be clinically more relevant than a single AHI measured in the sleep laboratory. We conclude that AHI measurements in the sleep laboratory are not routinely required to monitor effectiveness of CPAP therapy in OSA patients.

\section{0 \\ Prevalence of Sleep Disordered Breathing in the General Population According to Two Different Scoring Criteria}

\author{
J. Haba-Rubio, D. Andries, N. Tobback, F. Bastardot, \\ P. Vollenweider, M. Tafti, R. Heinzer
}

\section{CHUV, Lausanne}

Introduction: In studies from the 80's and 90's, the prevalence of sleep disordered breathing (SDB) in middle-aged general population is estimated at $9 \%$ in women and $27 \%$ in men. Considering the recent improvements in the sensitivity of recording techniques, our aim was to reevaluate the prevalence of SDB in the general population.

Methods: 505 subjects $(47.1 \%$ women, $50.3 \pm 5.6$ years old, BMI $25.7+/-4.4 \mathrm{~kg} / \mathrm{m}^{2}$ ) participating in an ongoing populationbased cohort study (HypnoLaus, Lausanne, Switzerland) underwent complete polysomnographic recordings at home and had an extensive clinical workup including Epworth Sleepiness score Score (ESS). Prevalence of SDB was determined according to apnea-hypopnea index (AHI) using two different scoring criteria: AASM 2007 and AASM 1999 'Chicago' criteria.

Results: With AASM 2007 criteria, prevalence of SDB with AHI thresholds of $5 / \mathrm{h}, 15 / \mathrm{h}$ and $30 / \mathrm{h}$ was $45.7 \%, 15,7 \%$, and $6.3 \%$, respectively in men, and $19.3 \%, 4.2 \%$, and $0.8 \%$ respectively in

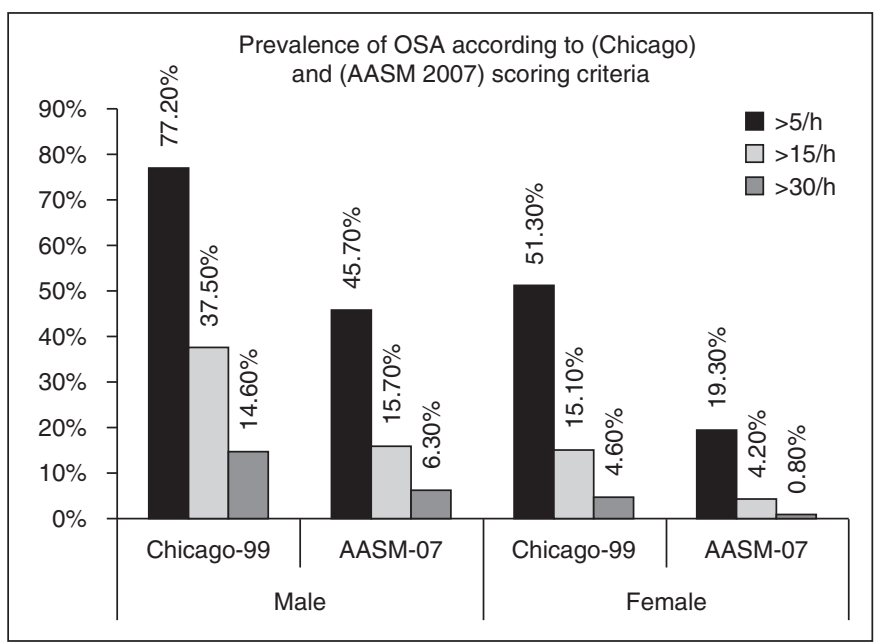

Fig. 1.

Respiration 2011;82:67-107 


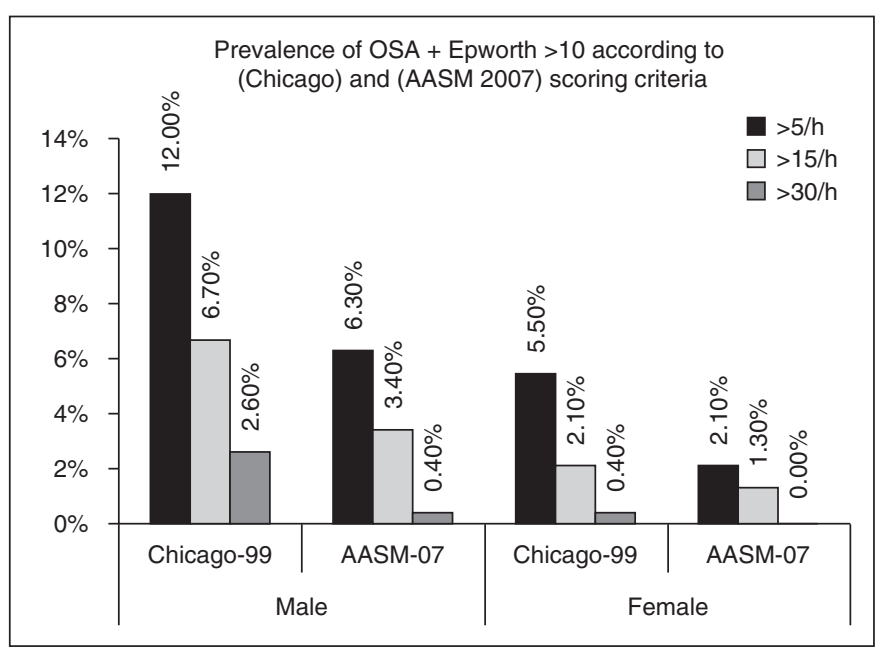

Fig. 2.

women. Mean ESS score was $6.9 \pm 4.2$ in men and $6.4 \pm 3.8$ in women. $18 \%$ of the men and $12.6 \%$ of the women had an ESS $>10$. The prevalence of ESS $>10$ and OSA with the same thresholds $(5 / \mathrm{h}$, $15 / \mathrm{h}$ and $30 / \mathrm{h}$ ) was $6.3 \%, 3.4 \%$, and $0.4 \%$, respectively in men and $2.1 \%, 1.3 \%$, and $0 \%$ in women. With AASM 1999 criteria, prevalence of OSA with the same thresholds was $77.2 \%, 37.5 \%$, and $14.6 \%$ in men and $51.3 \%, 15.1 \%$, and $4.6 \%$ in women. Prevalence of OSA and ESS $>10$ was $12.0 \%, 6.7 \%$, and $2.6 \%$ in men and $5.5 \%$, $2.1 \%$, and $0.4 \%$ in women.

Conclusion: In HypnoLaus population-based study, prevalence of SDB is much higher than previously reported, especially in middle-aged men. This appears to be due to differences in scoring criteria and to a higher sensitivity of nasal pressure sensors compared to thermocouples. These results warrant a reconsideration of the criteria for the definition of sleep disordered breathing.
61

\section{Are Sleep, Nocturnal Breathing and Daytime Performance of Lowlanders Impaired During a Stay at Moderate Altitude?}

T.D. Latshang ${ }^{1}$, C.M. Lo Cascio ${ }^{1}$, K. Stadelmann ${ }^{2}$, N. Tesler ${ }^{3}$, A.-C. Stöwhas ${ }^{1}$, P. Achermann ${ }^{2}$, R. Huber ${ }^{3}$, M. Kohler ${ }^{1}$, K.E. Bloch ${ }^{1}$

${ }^{1}$ University Hospital of Zurich, Zurich, ${ }^{2}$ Institute of Pharmacology and Toxicology, Zurich, ${ }^{3}$ Childrens Hospital, Zurich

Background: Many lowlanders feel that their sleep is disturbed during the first few nights of an altitude sojourn even at moderate elevation of Swiss alpine resorts. There are concerns that altitudeinduced sleep impairments and hypoxia might predispose to performance decrements during daytime. The current study evaluated the hypothesis that nocturnal sleep, breathing and psychomotor performance during daytime are disturbed in newcomers at moderate altitude.

Methods: 51 healthy males, mean \pm SD age $27 \pm 9 \mathrm{y}$, living at $<600 \mathrm{~m}$, were studied for 1 day/night at Zurich, for 2 days/nights at Davos Wolfgang (1630m) and Davos Jakobshorn (2590m), respectively. The order of stays at different altitudes was randomized. Polysomnographic recordings, psychomotor vigilance tests (PVT), snow board simulator tests and questionnaire evaluations were performed at all locations. The study was powered with $>80 \%$ to detect a 20ms change in PVT reaction time.

Results: Compared to $490 \mathrm{~m}$, sleep studies at altitude revealed reduced oxygen saturation associated with an increased central apnea/hypopnea index. Slow wave sleep was reduced and REM sleep increased at altitude while sleep efficiency and arousals revealed no consistent changes. PVT reaction time and snow board simulator racing time were not impaired at altitude compared to $490 \mathrm{~m}$ when controlled for learning effects in multiple logistic regression analyses. In the morning after polysomnography, Karolinska sleepiness scores and visual analogue scores of sleep quality were not consistently affected at altitude. Data are summarized in the table.

Table 1. Sleep studies and daytime evaluations (for Abstract 61)

\begin{tabular}{|c|c|c|c|c|c|c|}
\hline & \multirow{2}{*}{$\begin{array}{l}\text { Zurich } \\
490 \mathrm{~m}\end{array}$} & \multicolumn{2}{|c|}{ Davos Wolfgang, 1630 m } & \multicolumn{2}{|c|}{ Davos Jakobshorn, 2590 m } & \multirow{2}{*}{$\begin{array}{l}\mathrm{P} \\
\text { ANOVA }\end{array}$} \\
\hline & & 1st night & 2nd night & 1st night & 2nd night & \\
\hline $\mathrm{SpO}_{2}, \%$ & $96(95 ; 96)$ & $94(93 ; 95)$ & $94(93 ; 95)$ & $90(89 ; 91)$ & $91(90 ; 92)$ & $<10^{-5}$ \\
\hline AHI total, $1 / \mathrm{h}$ & $4.5(2.3 ; 7.9)$ & $7.2(4.1 ; 12.6)$ & $5.3(3.5 ; 10.5)$ & $12.7(6.7 ; 29.0)$ & $7.9(4.4 ; 20.5)$ & $<10^{-5}$ \\
\hline AHI central, 1/h & $2.0(1.2 ; 3.7)$ & $4.6(2.3 ; 7.9)$ & $2.8(1.7 ; 4.9)$ & $8.8(5.0 ; 25.1)$ & $5.4(2.9 ; 11.8)$ & $<10^{-5}$ \\
\hline Slow wave sleep, \% & $24(20 ; 27)$ & $24(19 ; 26)$ & $24(21 ; 29)$ & $20(17 ; 24)$ & $21(18 ; 25)$ & $<10^{-5}$ \\
\hline REM sleep, \% & $19(16 ; 24)$ & $22(18 ; 26)$ & $26(21 ; 29)$ & $20(18 ; 25)$ & $23(19 ; 27)$ & $<10^{-4}$ \\
\hline Sleep efficiency \% & $95(90 ; 97)$ & $95(88 ; 97)$ & $96(94 ; 97)$ & $95(91 ; 97)$ & $96(93 ; 98)$ & NS \\
\hline Arousal index, $1 / \mathrm{h}$ & $8.3(6.1 ; 9.5)$ & $6.5(5.3 ; 8.6)$ & $6.7(5.5 ; 8.3)$ & $7.6(6.1 ; 9.7)$ & $7.9(6.1 ; 10.9)$ & $<10^{-2}$ \\
\hline *Snowboard, sec & $37(35 ; 41)$ & $38(34 ; 41)$ & $37(33 ; 38)$ & $39(35 ; 41)$ & $37(33 ; 40)$ & $<10^{-3}$ \\
\hline${ }^{\star} \mathrm{PVT}, \mathrm{ms}$ & $203(183 ; 225)$ & $200(184 ; 229)$ & $196(181 ; 218)$ & $198(181 ; 227)$ & $195(179 ; 212)$ & 0.03 \\
\hline${ }^{*}$ Sleep quality, mm & $6.3(4.0 ; 7.5)$ & $6.0(4.3 ; 7.4)$ & $7.1(6.0 ; 8.2)$ & $5.6(3.9 ; 7.0)$ & $6.3(5.1 ; 7.9)$ & $<10^{-2}$ \\
\hline${ }^{*}$ Sleepiness & $3(2 ; 4)$ & $3(3 ; 5)$ & $3(3 ; 5)$ & $3(3 ; 5)$ & $3(3 ; 4)$ & NS \\
\hline
\end{tabular}

Values are medians (quartiles). $\mathrm{SpO}_{2}$ : nocturnal oxygen saturation, $\mathrm{AHI}$ : apnea/hypopnea index.

*evaluation in the morning after polysomnography. Snowboard simulator racing time: PVT psychomotor vigilance test reaction time; sleep quality: visual analogue scale from $0=$ extremely poor to $10=$ excellent; Sleepiness: Karolinska sleepiness scale, from $1=$ very awake to $9=$ very sleepy. 
Conclusion: In healthy young men, a stay at moderate altitude for a few days is associated with a slightly reduced nocturnal oxygen saturation, periodic breathing during sleep and subtle changes in sleep architecture. However, exposure to moderate hypobaric hypoxia does not alter subjective perception of sleep quality and daytime sleepiness nor does it consistently alter psychomotor vigilance.

Grant support: Zurich Center for Integrative Human Physiology, Schweizerische Unfallversicherungsgesellschaft.

\section{Free Communications 4 Clinical Research: Various Topics}

\section{2 \\ Monitoring of Leaks and Tidal Volume by Built-in Software of Home Bi-level Ventilators: A Bench Study \\ O. Contal', L. Vignaux' ', C. Combescure' ${ }^{1}$ J.-L. Pépin ${ }^{2}$, P. Jolliet ${ }^{3}$, J.-P. Janssens ${ }^{1}$}

${ }^{1}$ University Hospital of Geneva, Geneva, ${ }^{2}$ University Hospital of Grenoble, Grenoble, $\mathrm{F}^{3} \mathrm{CHUV}$, Lausanne

Background: Recent bi-level ventilators designed for home care provide the clinician with built-in softwares which monitor a large number of parameters such as compliance, estimation of leaks, tidal volume (VT), minute ventilation, apnea and hypopnea index, and cycles triggered by the patient. The present study aims to determine the reliability of assessment of VT and leaks by ventilator software.

Methods: Bench test evaluation of 7 home bi-level ventilators (Monnal T30 $^{\text {Ts }}$ (Airox; Pau, France); Synchrony ${ }^{\mathrm{Tm}}$ and Trilogy ${ }^{\mathrm{Tm}}$ (Respironics; Murrysville, PA); Ventimotion ${ }^{\mathrm{TM}}$ (Weinmann; Hamburg, Germany); Vivo 40 (Breas; Mölnycke, Sweden); VPAP III ST ${ }^{\mathrm{m} x}$ and VPAP IV ST-A ${ }^{\mathrm{m}}$ (ResMed; North Ryde, Australia). Calibrated leaks were generated by a computer-driven solenoid valve between the mask and ventilator. Three pneumotachographs recorded effective ventilation, and intentional (i.e. mask valve) as well as unintentional leaks (solenoid valve). Results were compared with data provided by ventilator software. Settings for tested ventilators were: EPAP: $5 \mathrm{cmH} 2 \mathrm{O}$; back-up frequency: 10/min; triggers and cycling set at default values. Tests were performed at 2 values of IPAP: 15 and $25 \mathrm{cmH} 2 \mathrm{O}$, with five different levels of leaks $(0,10,24,40,60 \mathrm{~L} / \mathrm{min})$.

Results: Table 1 summarizes results of Bland \& Altman assessment of Leaks and VT. Bias for leaks (all conditions pooled) ranged from 0.3 to $-25.9 \mathrm{~L} / \mathrm{min}$ according to the device tested. Three devices provided a reliable estimation of leaks, while 3 were intermediate, and one unreliable. VT (all conditions pooled) was underestimated by all devices with a bias which ranged from 66 to $236 \mathrm{ml}$, and was little affected by increasing leaks, but increased significantly with increasing IPAP pressure.

Conclusions: Estimation of leaks provided by home bi-level ventilators $1 /$ is not calculated or expressed in the same way for all devices tested, and 2/ yields highly variable results from one device to another. Tidal volume was underestimated by all devices; bias was little affected by leaks, but increased with higher insufflations pressure; underestimation of VT could be misleading for the clinician with some ventilators.

63

EGFR and KRAS Exonic Expression Profiling in Small Bronchoscopic Biopsies from Patients with Advanced Non-small Cell Lung Cancer Treated by Combined Bevacizumab/Erlotinib Therapy: A Multicentre Phase II Trial SAKK 19/05

M.H. Brutsche ${ }^{1}$, M. Früh', S. Crowe ${ }^{2}$, K.J. Na ${ }^{2}$, C. Dröge ${ }^{3}$, D. Betticher', R. von Moos ${ }^{5}$, F. Zappa ${ }^{6}$, M. Pless' ${ }^{7}$, L. Bubendorf ${ }^{3}$, F. Baty ${ }^{1}$

${ }^{1}$ Cantonal Hospital of St. Gallen, St. Gallen, ${ }^{2}$ SAKK Coordinating Center, Berne, ${ }^{3}$ University Hospital Basel, Basel, ${ }^{4}$ Cantonal Hospital of Fribourg, Fribourg, ${ }^{5}$ Cantonal Hospital of Graubünden, Chur, ${ }^{6}$ Oncology Institute of Southern Switzerland, Lugano, ${ }^{7}$ Cantonal Hospital of Winterthur, Winterthur

Background: The activity of bevacizumab/erlotinib (BE) was investigated in 101 treatment-naïve non-squamous stage IIIB and IV non-small cell lung cancer (NSCLC) patients in a single arm phase II trial (SAKK 19/05). We explored the effect of BE focusing on the exon intensity levels of EGFR and KRAS, a target and a prognostic gene.

Table 1. (for Abstract 62)

\begin{tabular}{|c|c|c|c|c|c|c|}
\hline Ventilator & $\begin{array}{l}\text { Average leaks } \\
(\mathrm{SD})(\mathrm{L} / \mathrm{min})\end{array}$ & Bias (SD) (L/min) & $\begin{array}{l}\text { Upper and lower limits } \\
\text { of agreement }(\mathrm{L} / \mathrm{min})\end{array}$ & $\begin{array}{l}\text { Average VT (SD) } \\
(\mathrm{ml})\end{array}$ & Bias (SD) (ml) & $\begin{array}{l}\text { Upper and lower limits } \\
\text { of agreement }(\mathrm{ml})\end{array}$ \\
\hline $\begin{array}{l}\text { Monnal T30 } \\
\text { Synchrony }\end{array}$ & $\begin{array}{l}63.8(10.9) \\
50.5(11.9)\end{array}$ & $\begin{array}{l}-8.3(6.1) \\
-6.0(3.2)\end{array}$ & $\begin{array}{l}{[-20.1 ; 3.6]} \\
{[-12.3 ; 0.3]}\end{array}$ & $\begin{array}{l}658(172) \\
696(228)\end{array}$ & $\begin{array}{r}130(46) \\
84(39)\end{array}$ & $\begin{array}{l}{[41 ; 220]} \\
{[7 ; 160]}\end{array}$ \\
\hline $\begin{array}{l}\text { Trilogy } \\
\text { Ventimotion } \\
\text { Vivo } 40\end{array}$ & $\begin{array}{l}58.5(13.6) \\
58.2(20.8) \\
54.8(10.5)\end{array}$ & $\begin{array}{c}0.3(1.0) \\
-16.3(5.2) \\
-25.9(12.7)\end{array}$ & $\begin{array}{l}{[-1.7 ; 2.3]} \\
{[-26.4 ;-6.1]} \\
{[-50.8 ;-1.0]}\end{array}$ & $\begin{array}{l}706(223) \\
809(258) \\
624(208)\end{array}$ & $\begin{array}{l}66(40) \\
236(125) \\
134(30)\end{array}$ & $\begin{array}{l}{[-13 ; 145]} \\
{[-9 ; 480]} \\
{[75 ; 193]}\end{array}$ \\
\hline VPAPIII & $14.6(12.7)$ & $0.8(1.5)$ & {$[-2.1 ; 3.7]$} & $789(267)$ & $174(61)$ & {$[53 ; 293]$} \\
\hline VPAPIV & $14.8(12.5)$ & $0.9(0.9)$ & {$[-0.8 ; 2.7]$} & $822(311)$ & $106(75)$ & {$[-41 ; 255]$} \\
\hline
\end{tabular}


Methods: The exonic expression profiling of 42 bronchoscopic biopsies was investigated using Affymetrix exon arrays. Unsupervised multivariate approaches, including principal component analysis (PCA) and hierarchical cluster analysis were used to describe the exonic variations within EGFR and KRAS among patients having various EGFR/KRAS mutational status. All analyses were blinded for the mutational status and clinical outcome.

Results: This analysis targeted all probe sets measured within EGFR $(n=451)$ and KRAS $(n=261)$. Fifty-one and 11 exonic probe sets were available within EGFR and KRAS respectively. PCA revealed a gradient of expression among patients which was homogeneous among the different EGFR exons (average withinpatient coefficient of variation $=0.28$, whereas larger exonic variations were found within KRAS (average within-patient coefficient of variation $=0.43$ ). In contrast to KRAS, there was a trend showing an association between gender and the expression of EGFR $(\mathrm{p}=0.06)$.

Conclusion: Using the exon array technology, it was possible to assess the exonic expression profiling of 2 key genes involved in non-squamous NSCLC growth pathways. EGFR expression level was homogeneous among exons, advocating independence between the exon intensity level and the patient mutational status. Conversely, KRAS proved to exhibit larger within-patient variation in the exonic expression level. We hypothesize that the exonic heterogeneity within KRAS provides additional prognostic information complementary to the patients' mutational status. These results will be provided after the unblinding of the trial.

\section{4}

\section{High-Throughput Gene Profiling of the Immediate Response to Bevacizumab/Erlotinib (BE) in Non- Squamous Non-small Cell Lung Cancer (NSCLC)}

F. Baty ${ }^{1}$, M. Früh ${ }^{1}$, S. Crowe ${ }^{2}$, K.J. Na ${ }^{2}$, C. Dröge ${ }^{3}$, D. Betticher ${ }^{4}$, R. Cathomas ${ }^{5}$, F. Zappa ${ }^{6}$, M. Pless ${ }^{7}$, M.H. Brutsche ${ }^{1}$

${ }^{1}$ Cantonal Hospital of St. Gallen, St. Gallen; ${ }^{2}$ SAKK Coordinating Center, Berne, ${ }^{3}$ University Hospital Basel, Basel, ${ }^{4}$ Cantonal

Hospital of Fribourg, Fribourg, ${ }^{5}$ Cantonal Hospital of Graubünden, Chur, ${ }^{6}$ Oncology Institute of Southern Switzerland, Lugano, ${ }^{7}$ Cantonal Hospital of Winterthur, Winterthur

Background: It is crucial to better understand the mechanisms of action of new therapies in advanced lung cancer. The SAKK investigated the response to $\mathrm{BE}$ on disease stabilization at week 12 in non-squamous NSCLC patients in a single arm phase II trial (SAKK 19/05). In the current translational research project, we investigated the $24 \mathrm{~h}$-effect of the combined BE therapy with the aid of whole blood transcriptomics.

Methods: The gene expression level of 43 out of 101 treatment naive non-squamous stage IIIB and IV NSCLC patients who received $\mathrm{BE}$, was profiled at $24 \mathrm{~h}$ and compared to baseline.The within patient gene variations were analyzed using multivariate between-group correspondence analysis (BGA) together with univariate paired t-tests. All analyses were blinded for the patients' mutational status and clinical outcome.
Results: The 24h-gene dysregulation due to BE was limited, showing 638 out of $26^{\prime} 116(2.4 \%)$ significant variations at the nominal level $(\mathrm{p}<0.05)$. Functional analysis showed a significant enrichment of dysregulated genes involved in pathways such as hematopoietic cell lineage, cytokine-cytokine receptor interactions, T-cell receptor signaling and cell adhesion molecules. The gene encoding for the matrix metalloproteinase 9 (MMP-9) was top ranked in the lists obtained both by gene-by-gene and multivariate approaches. MMP9 showed a down-regulation after $24 \mathrm{~h}$ $(\mathrm{p}=0.02)$. The $24 \mathrm{~h}$-response varied among patients, $72 \%$ of patients showing a down-regulation of MMP9.

Conclusion: The 24h-effect of BE, as measured in the blood, induced the regulation of a limited, yet consistent number of genes among which MMP-9. This gene, which is involved in the pathogenesis of metastatization, may be a potential early marker of clinical response to BE therapy. After unblinding for clinical outcome it is planned to identify potential early markers of 12 -week disease stabilization, tumor response and overall survival with BE.

\section{5 \\ Kidney Transplantation Improves Lung Function in Lung Transplant Recipients}

\author{
M. Schuurmans, G.M. Tini, C. Benden, M.K. Mueller, T. Fehr, \\ A. Boehler \\ University Hospital of Zurich, Zurich
}

Background: Chronic kidney disease is a major co-morbidity following lung transplantation, particularly caused by calcineurin inhibitor (CNI) toxicity. Kidney transplantation in previous lung transplant recipients is considered the ultimate therapy option, however, only performed in a relatively small number of patients so far. We aim to evaluate outcomes of kidney after lung transplants (KAL) at our centre in terms of lung and kidney graft function.

Methods: A retrospective, single-center review was performed, including KAL transplant recipients undergoing renal transplants between 2002 and 2009.

Results: In more than 250 lung transplant recipients, 7 KAL transplants were performed ( 3 women), 5 from living donors. Patients underwent lung transplantation for cystic fibrosis (4), idiopathic pulmonary fibrosis (2) and pulmonary arterial hypertension (1) (1995-2002). Mean time to kidney transplantation was 104.7 months after lung transplant. No renal grafts were lost during follow-up, all renal grafts are functioning with a mean followup of 38 months. Complications after kidney transplantation included urinary tract infections (3/7), with 1 patient requiring a uretero-pyelostomy. Patients experienced a mean FEV1 decline of $22 \mathrm{~mL} / \mathrm{month}$ (range 6-42) over a 12-month period prior to the kidney transplant (Bronchiolitis Obliterans Syndrome (BOS) grade $0, \mathrm{~N}=1$; BOS grade $0-\mathrm{p}, \mathrm{N}=2$; BOS grade $1, \mathrm{~N}=4$ ). Thereafter, lung function overall stabilized in 5/7 recipients; moreover, FEV 1 actually improved in most recipients. Mean increase of FEV1 was $3.6 \%$ and of FVC was $5.9 \%$ by 1 -year post kidney transplant, with further increases to $9.9 \%$ and $12.3 \%$, respectively. All 7 patients have survived to date. 
Conclusion: Our findings show good outcomes of kidney transplantation in KAL recipients. Moreover, lung function stabilized and even improved in selected patients. This information may be helpful in clinical decision-making and organ allocation in this population.

\section{Poster Session 1 COPD/Asthma}

\section{P71}

Prediction of Exercise-Induced Oxygen Desaturation in Patients with Chronic Obstructive Pulmonary Disease

\author{
A.J.R. van Gestel' , C.F. Clarenbach ${ }^{1}$, G. Mettler ${ }^{1}$, S. Teschler ${ }^{2}$, \\ E.W. Russi ${ }^{1}$, H. Teschler ${ }^{2}$, M. Kohler ${ }^{1}$ \\ ${ }^{1}$ University Hospital Zurich, Zurich, ${ }^{2}$ University Hospital \\ Ruhrlandklinik, Essen, D
}

Background: Many patients with Chronic Obstructive Pulmonary Disease (COPD) experience increasing deterioration of oxygen saturation ( $\mathrm{SpO} 2$ ) during physical exercise which may be associated with increased risk of mortality. Previous studies with small sample sizes reported contradicting findings as to whether pulmonary function tests, such as forced expiratory volume in one second (FEV1) and diffusion capacity for carbon monoxide (DLCO), can predict exercise-induced oxygen desaturation (EID).

Objective: To evaluate whether FEV1 and DLCO are predictors of EID in COPD.

Methods: We measured FEV1 and DLCO as well as maximal percentage of oxygen desaturation during a 6-minute walking test in patients with COPD (GOLD stages I-IV). A drop in oxygen saturation of $>4 \%$ was defined as EID. To evaluate a possible association between measures of lung function and EID univariate and multivariate analyses were used and positive/ negative predictive values were calculated. Receiver operating characteristic curve (ROC) analysis was performed to determine the most useful threshold in order to predict/exclude EID.

Results: We included 154 patients with COPD (87 females) with a mean [SD] age of 62.5 [10.7] years and mean FEV1 \%pred. of 43.00 [19.24] \%; 113 of the $154(73.4 \%)$ patients had EID. The severity of EID was significantly correlated with FEV1 $(r=-0.56$, $\mathrm{p}<0.001)$ and DLCO $(\mathrm{r}=-0.49, \mathrm{p}<0.001)$. Including both FEV1 and DLCO in a multivariate analysis model, $33.0 \%$ of the variance in EID could be explained $(\mathrm{r} 2=0.33, \mathrm{p}<0.001)$. By including only FEV $1,32.0 \%$ of the variance in EID could be explained $(\mathrm{r} 2=0.32$, $\mathrm{p}<0.001)$ and estimated with the predictive formula: EID $(\%)=-$ 0.19 FEV1 (\% predicted) +16.6. The optimal cut-off value of FEV1 $\%$ pred. was at $50 \%$ (area under the ROC curve, 0.78 ; $\mathrm{p}<0.001$ ). The positive predictive value of a threshold of FEV $1<50 \%$ was

Abstracts

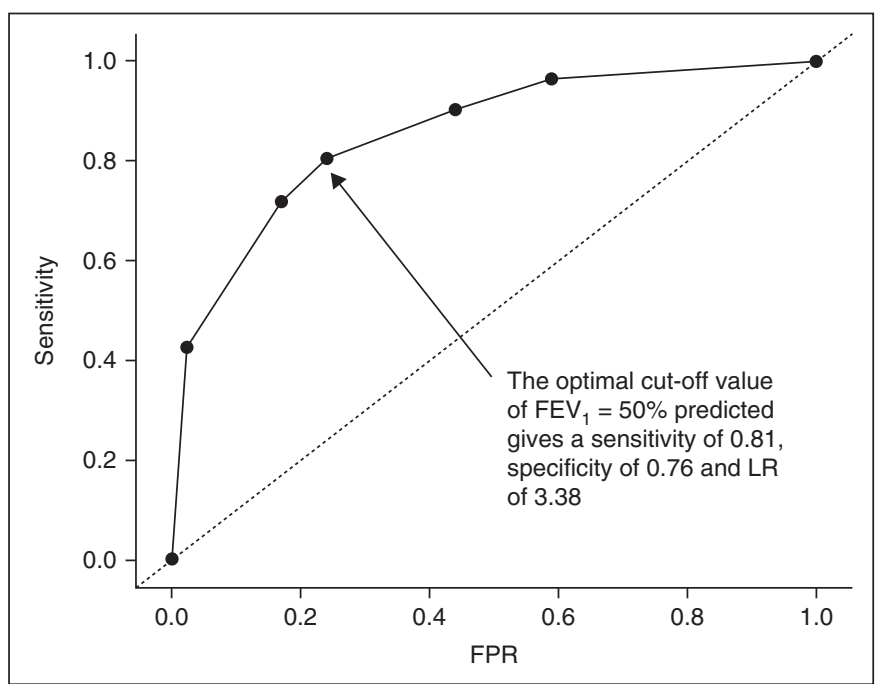

Fig. 1. Receiver operating characteristic curve (ROC) showing that the best combination of sensitivity and specificity occured at a FEV value of $50 \%$ (area under the ROC curve 0.78; p < 0.001). LR = likelihood ratio.

90.1\%. The negative predicting value of a threshold of FEV $1>80 \%$ was $86.0 \%$.

Conclusions: It is possible to estimate exercise-induced oxygen desaturation using FEV1, \%predicted. This finding may help to decide whether a 6-minute-walking test with measurement of $\mathrm{SpO} 2$ should be performed in patients with COPD.

\section{P72 \\ Factors Influencing the Utilisation of Hospital Resources in Patients with COPD in Switzerland}

B.D. Isenring, F. Baty, L. Kern, M.H. Brutsche

Cantonal Hospital of St. Gallen, St. Gallen

Introduction: Between 2002-2007, 3.1\% of all hospitalizations in $\mathrm{CH}$ contained COPD as diagnosis entity. In 0.6\%, COPD was coded as main diagnosis (predominantly because of exacerbated COPD [AECOPD]), and could be considered as the reason for hospitalization. The aim was to identify factors influencing the length of stay (LOS) and the time to rehospitalization (TTR), two factors reflecting the costs of hospitalized COPD.

Material and Methods: A database provided by the Swiss Federal Office for Statistics enclosing every hospital entry covering the years 2002-2007 was used. Data management and analysis were done using MySQL database and the R statistical software. Factors associated with time-to-event endpoints were analyzed using Kaplan-Meier estimators and the Andersen-Gill formulation of the cox-proportional hazards model.

Results: Patients older than 17, with COPD as main diagnosis were hospitalized for a median period of 11d (IQR: 7-17). The rehospitalization rate due to COPD as main diagnosis was $26 \%$

Respiration 2011;82:67-107 79 


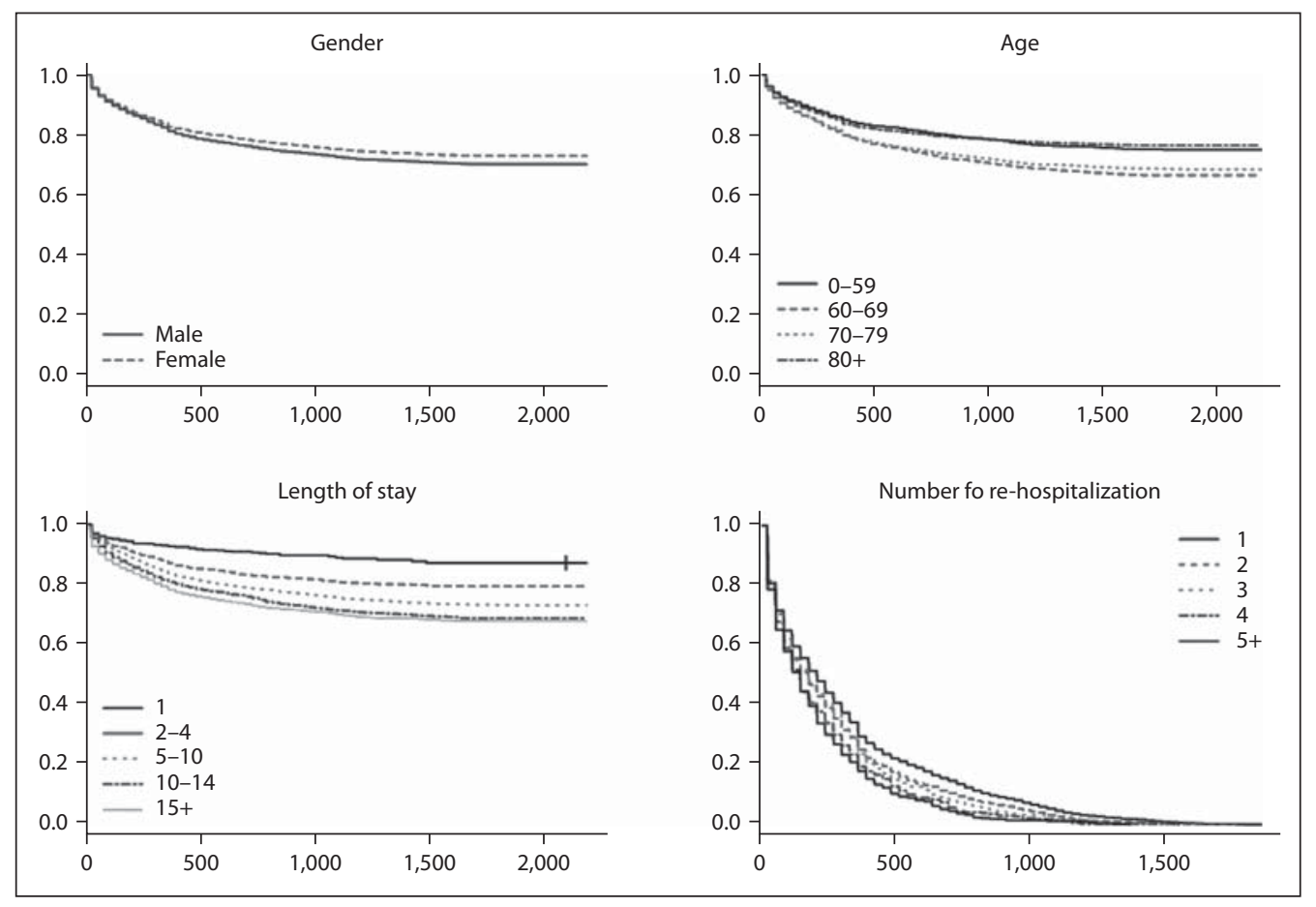

Fig. 1. (for Abstract P72)

(males: 27\%, females: 25\%). The median time between 2 hospitalizations was 5.0 months (IQR: 1.5 - 11.0). Factors influencing time to rehospitalization included gender with a shorter TTR in males $(\mathrm{p}<0.001)$, age with younger and older patients having a longer TTR $(\mathrm{p}<0.001)$, number of rehospitalization, the more often the shorter and finally the LOS with patients staying longer tending to be rehospitalized more rapidly (see Figure 1).

Conclusion: We could identify factors significantly affecting time to rehospitalisation, which occurs almost always within one year after first hospitalisation due to (AE) COPD.

As a next step we will test the associations between TTR/ LOS and our formerly (ERS 2010) identified comorbidity-clusters and factors influencing LOS.

\section{P73}

\section{Rethinking Models of Care to Improve Chronic Care in COPD Patients}

\section{Steurer-Stey ${ }^{1}$, K. DallaLana' ${ }^{1}$ S. Stoller ${ }^{2}$, A. Pfister ${ }^{2}$, O. Senn', M. Puhan ${ }^{3}$ \\ ${ }^{1}$ University of Zurich, Zurich, ${ }^{2}$ Medix Gruppenpraxis Zürich, Zürich, ${ }^{3}$ John Hopkins University, Baltimore, USA}

Background: The Chronic Care Model (CCM) is a successful strategy for primary care to improve care for patients with chronic illnesses. However, limited data exist in implementing and evaluating CCM elements in patients with chronic obstructive pulmonary disease (COPD).
We assessed the feasibility of two central CCM elements, self management support and delivery system design, for COPD patients in primary care.

Method: Our intervention focused on self-management support and a practice based multiprofessional care team approach. We assessed disease and patient specific aspects and offered proactive follow up. Six group modules (what is COPD; treatment and inhalation techniques, breathing techniques, energy conservation, physical activity, and a plan of action for exacerbation) addressed knowledge and skills necessary for COPD management.

Quality of life measured with the validated German version of the Chronic Respiratory Questionnaire (CRQ) and the perceived burden of disease measured with the Pictorial Representation of Illness and Self Measure (PRISM) were assessed before and after the intervention.

Results: All recruited patients $(n=25,52 \%$ male, $60 \%$ current smokers) completed the programme. Mean (SD) patients age and years of diagnosis were $65.6(11.8)$ and $6.2(4.7)$ years. Mean (SD) pulmonary function tests (FEV1, FEV1/FVC ratio) were 53.5 (19.0) \% predicted and 0.57 (0.14), respectively. Comorbidities were frequent ( $36 \%$ with cardiovascular disease).

A significant improvement in quality of life with a mean CRQ increase of 0.8 points (minimal clinical important difference 0.5 points) was measured. The median (IQR) self-illness separation (PRISM) increased from $9(5-22) \mathrm{cm}$ at the beginning to 24 (1826) $\mathrm{cm}$ after the intervention.

Conclusion: A care team approach and self-management support for COPD patients is feasible in primary care. Improved quality of life and reduced perceived burden of disease highlight the positive impact of a productive interaction between a prepared 


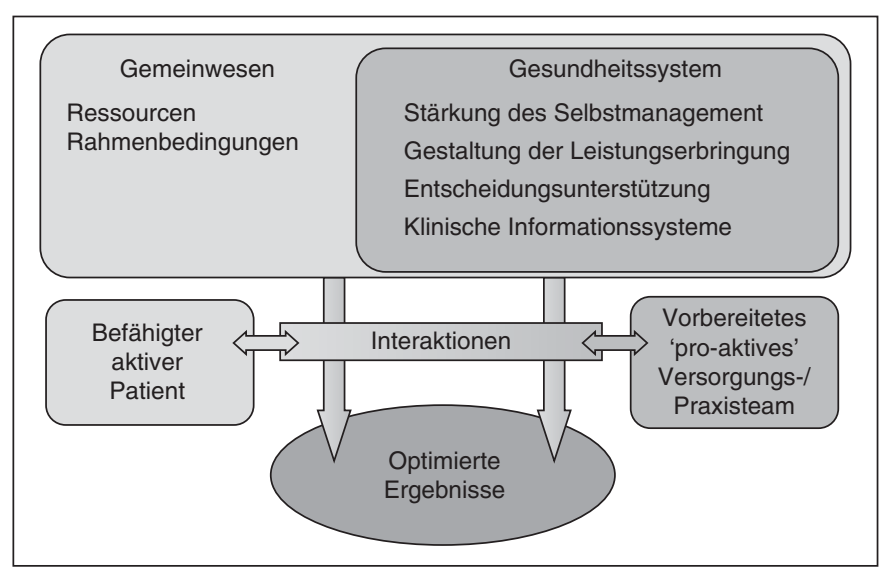

Fig. 1. Adaptation Steurer-Stey C. Department of General Practice and Health Services Research, University Zurich, Switzerland.

practice team and activated patients. To quantify the effects of our CCM congruent programme on patient important outcomes in COPD well designed trials are needed.

\section{P74 \\ Exertional Dyspnoea and Persistent Coughing - Exercised Induced Asthma?}

S. Diemer, K.-H. Stirner, A. Jung, C. Geidel, R. Lauener

Hochgebirgsklinik Davos, Davos

Introduction: Bronchial asthma is a common disease and it is diagnosed in 10 to $15 \%$ of all children and adolescents in Europe. However, other serious disorders can present with asthma-like symptoms.

Case history: $16 \frac{1 / 2}{2}$ year-old girl, since the age of 9 exertional dyspnoea and persistent cough with no history of allergy. Since 12 months progressive dyspnoea during exercise, perennial rhinitis and broncho-pulmonary impairment during exacerbations. Productive cough with yellow-white sputum, especially during the night. The patient reports about nocturnal in- and exspiratory stridor and about a noise like an opening and closing valve. Pulmonary function tests show reversible airway obstruction, normal FeNo and normal exercised induced asthma test. Beginning of treatment with ICS and LABA, and additionally LTRA without further improvement.

Admission to the pediatric department of Hochgebirgsklinik Davos with suspected diagnosis of EIA. Clinical investigations show an irreversible airway obstruction, normal FeNo and a mild house dust mite allergy. Upon auscultation, prolonged exspirium and an in-and exspiratory wheezing-like sound over trachea and larynx. Bronchoscopy revealed signs of subacute bronchitis and a pediculated, almost occlusive tumor in the left main bronchus, 3 $\mathrm{cm}$ distal of the carina. Thoracic CT scan showed a $1 \mathrm{~cm}$ in diameter, endoluminal tumor of the left main bronchus with contrast enhancement. Suspected diagnosis: carcinoid or adenoid cystic carcinoma. In the following segment resection of the left main bronchus with bronchus anastomosis and lymphadenectomy. Pathology confirmed a well-differentiated neuroendocrine carcinoid tumor.

Conclusion: Exertional dyspnoea and persistent cough are typical symptoms of bronchial asthma. However, in case of persistent symptoms resistant to treatment, other respiratory and non-respiratory diseases should be eliminated by further diagnostic investigations.

\section{P75 \\ The Drifter - A Neglected Phenotype of Asthma}

T. Rothe

\section{Zürcher Höhenklinik Davos, Davos}

Background: Asthma phenotypes are object to intense research. Being able to better phenotype asthma may help to find specific therapies. A 'drifter' type of asthma had been described by M. Turner-Warwick in 1977. Here patients had presented with progressive bronchial obstruction without reversibility to beta-agonists (BA). However, her drifter type of asthma showed reversibility to corticosteroids.

Methods: 2 male patients (M.S., 48 y and B.P., 60y) presented with marked bronchial obstruction without significant reversibility to short acting (SA)-BA. Due to suspicion of asthma they received an oral steroid trial of $40 \mathrm{mg}$ for 14 days.

Results: Both patients suffered from exertional dyspnoea, not from acute attacks. M.S. had a history of 30 py of cigarettes. Progressive obstruction occurred while he was not on anti-asthma drugs. In B.P. non-reversible obstruction progressed despite a combined therapy of long acting (LA)-BA and topical steroids. He was a lifelong non-smoker. Prior to the oral steroids he received a bronchoscopy revealing glue-like mucus plugging of subsegmental bronchi. Cytologic examination showed accumulation of eosinophils and multiple Curschman spirals.

In both cases FeNO values were elevated indicating eosinophilic inflammation of the airways. Histories and skin prick testing did not reveal any extrinsic cause of the bronchial inflammation. Initially, M.S. showed a post-bronchodilator FEV 1 of $48 \%$ of normal, B.P. of $73 \%$. FEV1 values after treatment with oral corticosteroids improved to $114 \%$ in M.S. and to $110 \%$ of normal in B.P.

Being of Chinese origin, M.S. did not understand well the medical advice and did not continue with topical steroids and LABA after the steroid trial. At a visit 2 weeks later the post-bronchodilator FEV1 had dropped for 0.63 litres. Again, the obstruction was not reversible to SABA.

Conclusion: 2 patients with a drifter type of asthma according to Turner-Warwick are presented. Especially in M.S., a former smoker, this phenotype is difficult to differentiate from COPD. In drifter asthma symptoms are dominated by progressive exertional dyspnoea without acute attacks. Typical asthma features of lung function as reversibility to SABA and hyperinflation are lacking. In case of a newly diagnosed irreversible bronchial obstruction elevated FeNO and/or blood respectively sputum eosinophilia should prompt a systemic steroid trial in order to not oversee the drifter type of asthma. 


\section{P76}

Efficacy of Roflumilast in Patients Receiving Concomitant Treatments for Chronic Obstructive Pulmonary Disease Over 12 Months

\section{A. Gooss ${ }^{1}$, H. Rusch ${ }^{2}$, T.D. Bethke ${ }^{2}$, N. Hanania ${ }^{3}$}

${ }^{1}$ Nycomed Pharma AG, Dübendorf, ${ }^{2}$ Nycomed Deutschland GmbH, Constance, D, ${ }^{3}$ Baylor College Medicine, Houston, USA

Background: The oral, selective phosphodiesterase 4 inhibitor roflumilast improves lung function and reduces exacerbations in patients with severe-to-very severe chronic obstructive pulmonary disease (COPD) and improves lung function and other clinical outcomes in patients with moderate-to-severe COPD who are receiving concomitant long-acting inhaled bronchodilators. We analyzed data from two 12-month studies that investigated the effect of roflumilast on the rate of moderate or severe exacerbations in patients with severe-to-very severe COPD. Our aim was to investigate whether the effect of roflumilast is influenced by concomitant maintenance treatment with bronchodilators (longacting beta2 agonist [LABA] or short-acting muscarinic antagonist [SAMA]) or by treatment with inhaled corticosteroid (ICS) prior to randomization.

Methods: M2-124 and M2-125 were identically designed, double-blind, randomized studies. Patients with severe-to-very severe COPD associated with cough and sputum production (chronic bronchitis) and a history of exacerbations were randomly assigned to either roflumilast $500 \mu \mathrm{g}$ once daily $(\mathrm{n}=1537)$ or placebo once daily $(n=1554)$ for 12 months. Concomitant LABA and regular SAMA use were allowed in all groups, but ICS was discontinued at randomization.

Results: LABAs were used by 749 (49\%) patients in the roflumilast group and 793 (51\%) patients in the placebo group in the pooled data set. The corresponding numbers for regular SAMA use were 537 (35\%) and 569 (37\%), respectively; 650 (42\%) and 657 (42\%), respectively, were treated with ICS prior to randomization. The change in exacerbation rate with roflumilast vs placebo was $-16.9 \%$ (95\% confidence interval $[\mathrm{CI}]-25,-8)$ in the overall population and was not influenced by LABA use, with changes in exacerbation rates of $-20.7 \%(95 \%$ CI $-31,-9)$ and $-14.6 \%(95 \%$ CI $-26,-1)$ in the subgroups of LABA users and non-LABA users, respectively. The corresponding changes in exacerbation rates for concomitant SAMA use vs non-SAMA use were -13.1\% (95\% CI $-24,-1)$ and $-19.8 \%(95 \%$ CI $-30,-8)$, respectively. In patients with and without prior ICS treatment, exacerbations changed by $-19.3 \%(95 \%$ CI $-30,-7)$ and $-16.8 \%$ (95\% CI $-28,-4)$, respectively, with roflumilast vs placebo.

Conclusions: In two 12-month studies, roflumilast reduced the frequency of moderate and severe exacerbations independent of concomitant maintenance bronchodilator treatment (LABA or SAMA), or treatment with ICS prior to randomization.

\section{P77 \\ Vocal Cord Dysfunction - A Rare But Important Differential Diagnosis of Asthma in Childhood}

\author{
S. Diemer, I. Heinrichs, A. Jung, C. Geidel, R. Lauener \\ Hochgebirgsklinik Davos, Davos
}

Introduction: Vocal cord dysfunction is a disorder of the vocal cords and leads to an obstruction of the upper airways. This obstruction can mimic bronchial asthma. It is important to recognize vocal cord dysfunction as a differential diagnosis of bronchial asthma in order to avoid unnecessary anti-asthmatic therapy.

Case report: 10 year-old obese boy, since the age of 4 years diagnosis of non-atopic asthma. During the last two years recurrent episodes of sudden dyspnoea, subsequent consultation of different physicians. Despite intensive inhaled anti-asthmatic therapy and several intervals of systemic steroids no improvement of frequency or severity of dyspnoea episodes. Symptoms occur mainly during exercise and are accompanied by chest tightness and inspiratory stridor. During the last year three admissions to the emergency department after exercise. Further clinical investigations including X-ray, sweat test and testing for infectious diseases yielded no pathologic results. Consequently, the patient avoided any physical activities. Admission to the pediatric department of Hochgebirgsklinik Davos for pulmonary rehabilitation. Pulmonary function tests such as body plethysmography, exhaled nitric oxide and methacholine challenge test without pathological findings. However, during exercise induced asthma test and social-pedagogical program with physical exercise repeated dyspnoea episodes with inspiratory stridor. Spontaneous improvement of these episodes without inhaled therapy. Clinical picture suggested vocal cord dysfunction. Laryngoscopy during exercise was performed and gave evidence of sponteanous and exercise-induced laryngospasm. After patient education and breathing exercise from a physiotherapist the patient reported significant improvement in symptom control. The patient can now take part in physical activities again and hasn't had any dyspnoea episodes during the last six months.

Conclusion: Asthma is a common diagnosis in children. However, when respiratory symptoms persist despite adequate treatment and good compliance, other diagnosis should be considered. Pulmonary rehabilitation programs can be helpful to initiate a broad diagnostic work-up, and subsequently to enhance disease management.

\section{P78 \\ Diffuse Aspiration Bronchiolitis. An Underestimated Diagnosis}

\section{J.J. Rüdiger, L. Kern, M.H. Brutsche \\ Cantonal Hospital St. Gallen, St. Gallen}

A 64 year old private practitioner was seen in the pulmonary clinic because of excertional dyspnea, progredient coughing for months and hemoptysis since serveral weeks. Besides an upside down stomach, causing severe reflux esophagitis his past medical 

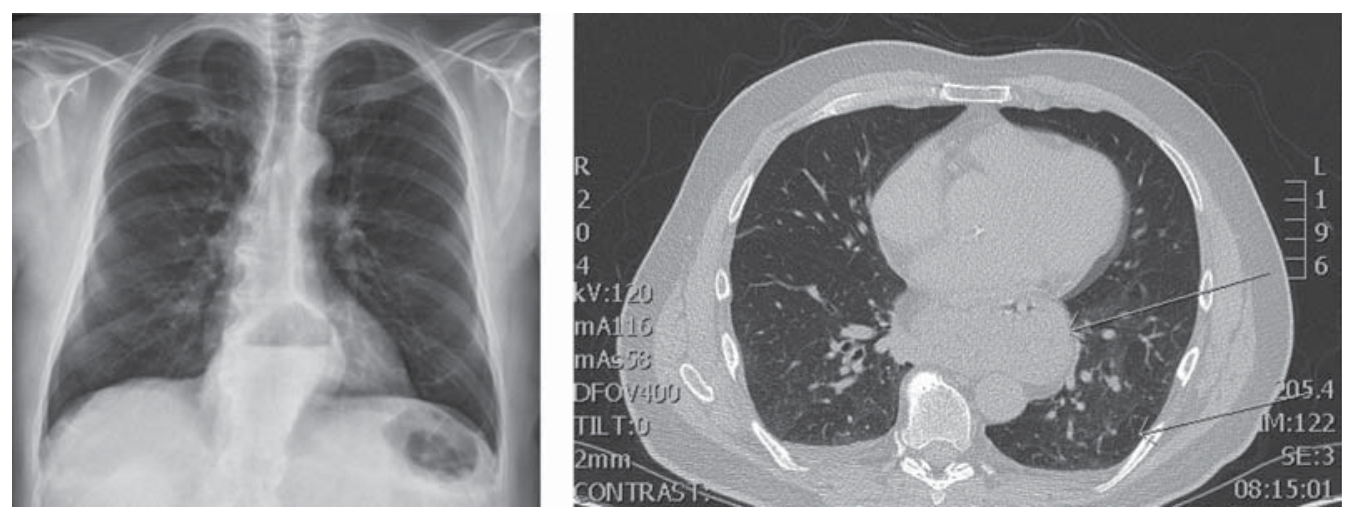

Fig. 1. (for Abstract P78)

history was unremarkable. He has been seen by an experienced gastroenterologist and treated with high dose proton pump inhibitors for longer than 2 monthes. He denies shortness of breath at rest, night sweats, fever or weight loss. Mantoux testing was negative. Serum was negative for anti-basement membrane antibodies, ANA, ANCA and rheumatoid factors. Hematocrit was within normal limits. Physical examination was normal as well chest X-ray. Lung function showed normal dynamic and static lung volumes. Diffusion capacity for CO was within normal limits.

We decided to perform bronchoscopy. CT-scan just previously to the bronchoscopy CT-scan was considered normal by a radiologists, to us there appeared to be a faint nodular pattern in the distal inferior lung and middle lobe. During bronchoscopy the mucosa of the entire bronchial system showed marked inflammation and a localized bleeding mucosal lesion within the middle lobe bronchus. There was absence of prurid discharge.

Bronchoalveolar lavage within the middle lobe was negative for tuberculous species in PCR and culture, regular cultures reveald low amounts of oral flora. Cytologic evaluation resulted in normal cell count and differential, there were, however, a few iron ladden macrophages.

Because of the severity of inflammation we thought of a reflux associated bronchiolitis and repeated the CT-scan in exspiration, showing a mosaic pattern with air trapping confirming the presence of bronchiolitis. Those findings were consistent with the diagnosis of diffuse aspiration bronchiolitis. In $\mathrm{pH}$-metric testing persistent reflux could then be documented. We suggested surgery fearing a progression of the bronchiolitis into an irreversible state and. After the persistent reflux suspected was confirmed the patient could be scheduled for surgery.

\section{Poster Session 2 \\ Basic Research/Tumour/Transplantation}

P79

\section{Lung on Chip: Co-culture of Alveolar Epithelial Cells and Bone Marrow Derived Stem Cells in a Microfluidic System}

\author{
A. Schoeni' , L. Barbe' ${ }^{2}$, A. Gazdhar' 1 , T. Geiser ${ }^{3}$, O. Guenat ${ }^{1}$ \\ ${ }^{1}$ University of Berne, Berne, ${ }^{2}$ CSEM, Landquart, ${ }^{3}$ University \\ Hospital of Berne, Berne
}

Background: Microfluidic systems are novel tools to study cellcell interactions in vitro. This project focuses on the development of a new microfluidic device to co-culture alveolar epithelial cells and mesenchymal stem cells to study cellular interactions involved in healing the injured alveolar epithelium.

Methods: Microfluidic systems in polydimethylsiloxane were fabricated by soft lithography. Human alveolar A549 epithelial cells were seeded and injury tests were made on the cells by the perfusion with media containing $\mathrm{H}_{2} \mathrm{O}_{2}(0.0625 \mu \mathrm{M}, 0.625 \mu \mathrm{M}, 1.25$ $\mu \mathrm{M})$ or bleomycin $(0.002 \mathrm{U} / \mathrm{ml}, 0.02 \mathrm{U} / \mathrm{ml}, 0.2 \mathrm{U} / \mathrm{ml})$ during 6 or 18h. Rat -bone marrow derived stem cells (BMSC) were then introduced into the system and cell-cell interaction was studied over 24 hrs.

Results: A successful co-culture of A549 alveolar epithelial cells and BMSC has been achieved in the microfluidic system. The seeded alveolar epithelial cells and BMSC adhered to the bottom surface of the microfluidic device and proliferated under constant perfusion. Epithelial injury to mimic mechanisms seen in a variety of lung diseases like for instance pulmonary fibrosis was induced in the microchannels by perfusing with $\mathrm{H}_{2} \mathrm{O}_{2}$ or bleomycin. Migration of BMSC towards the injured epithelium was observed as well as cell-cell interactions between the two cell types.

Conclusions: We demonstrate a novel microfluidic device aimed at mimicking the epithelial barrier in the alveolar space, showing interactions between different cell types on the basis of a 
changing microenvironment. Also we were able to confirm interactions between injured alveolar epithelium and BMSC. Further studies are needed to characterize the cell-cell interactions in microfluidic systems., and showed that BMSC try to heal the injured epithelium.

\section{P80 \\ HGF Expressing Stem Cells in the Human Fibrotic Lung Originate from the Bone Marrow}

N. Susuri ${ }^{1}$, A. Gazdhar ${ }^{1}$, M. Gugger ${ }^{1}$, T. Geiser ${ }^{2}$

${ }^{1}$ University of Berne, Berne, ${ }^{2}$ University Hospital of Berne, Berne

Background: Pulmonary fibrosis (PF) is a progressive disease of unknown etiology. Abnormal alveolar epithelial wound repair after injury may result in pulmonary fibrosis. We hypothesize that stem cells have a healing capacity by migrating to the site of injury and secreting hepatocyte growth factor (HGF) which supports alveolar epithelial repair, therefore contributing in reduction of fibrosis.

Methods: Immunohistochemistry using paraffin lung sections from patients with two histological lung fibrosis pattern, usual interstitial pneumonia (UIP) and non-specific interstitial pneumonia (NSIP) (both $\mathrm{n}=5$ ) was performed with several stem cell markers.

Results: Specific cells in the lung parenchyma were stained positive for HGF in UIP and NSIP. They were mainly located in the fibrotic areas. These HGF-positive cells did not co-stain for markers of alveolar epithelial cell (Surfactant protein C) or fibroblasts (vimentin). However, HGF-positive cells showed strong costaining for the mesenchymal stem cell markers CD44, CD29, CD105, and CD90, indicating that HGF positive cells in the lung are of stem cell origin. The HGF-positive cells were also positive for CXCR4, suggesting that the HGF-positive cells in UIP and NSIP are recruited from the bone marrow.

Conclusion: HGF-positive stem cells with the origin of the bone marrow (CXCR4 positive) can be detected in both UIP and NSIP, indicating a crucial role in the development or resolution of pulmonary fibrosis. These data indicate a possible role for stem cell therapy of patient with fibrotic lung disease in the future.

\section{P81 \\ Commercial Surfactant Modulates Dendritic Cell Phenotype and Functionality}

M. Huguenot, F. Blank, T. Geiser, C. von Garnier

University Hospital of Berne, Berne

Background: With its exquisitely delicate architecture, the lung is a highly vulnerable organ to inhaled, potential harmful antigen. Maintenance of local immunological homeostasis and hence the integrity of the gas-exchange surfaces stretches the discriminatory powers of the immune system to their limits. To maintain immu- nological homeostasis the respiratory tract is endowed with a high density of immune cells (e.g. dendritic cells, DC) that underlie stringent control mechanisms. Pulmonary surfactant, well known for its surface tension reducing properties, has also been shown to exhibit very important immunoregulatory properties, largely governed by the two hydrophilic surfactant proteins SP-A and SP-D. As commercial surfactants are obtained by lipid-extraction, they contain no hydrophilic proteins but only SP-B and SP-C. The aim of this study was to monitor the immunomodulatory functions of the commercial surfactant Curosurf (CS) using an in vitro model of human pulmonary dendritic cells.

Methods: We used in vitro differentiated human blood monocytes derived dendritic cells (MDDC) exposed CS (250ug/ml) for $12 \mathrm{~h}$ in the presence or absence of lipopolysaccaride (LPS). We analysed the expression pattern of specific surface markers and the functionality (antigen uptake (Alexa Fluor conjugated ovalbumin) and processing (DQ-ovalbumin) capacities) by flow cytometry and laser scanning microscopy.

Results: We observed decreased antigen uptake capacity in MDDC exposed to CS and LPS (MFI: 66\% of control) associated with an increase in MHC-II (MFI: $123 \%$ of control) and CD86 (MFI: $128 \%$ of control). Curosurf alone showed almost no effect in the absence of LPS.

Conclusion: Our data suggests that CS potentially induces maturation in MDDC, that display less antigen uptake, and up-regulate both co-stimulatory molecules (e.g. CD86) and MHC-II in a time dependent manner. Further experiments are required to confirm the CS-induced maturation focusing on the modulation of downstream immune responses such as $\mathrm{T}$ cell stimulation and cytokine production.

\section{P82 \\ Systemic Distribution of Biomedical Nanoparticles Applied via the Pulmonary Route}

R. Vogel, F. Blank, A. Gazdhar, H.R. Widmer, T. Geiser, C. von Garnier

University Hospital of Berne, Berne

Background: Research into biomedical nanoparticles (NP) for novel diagnostic or therapeutic applications has been constantly increasing over the last years. Monitoring NP-induced effects on the immune system and distribution in the organisms is essential for the further development of novel drug and vaccine nanocarriers. In the present project we aimed to follow the fate and the distribution of poly(vinylalcohol)-coated super-paramagnetic iron oxide NP (PVA-SPIONS; 50nm in diameter) delivered via the pulmonary route with a particular focus on the lung parenchyma and brain.

Methods: PVA-SPIONS were delivered intra-bronchially (leftsided intubation) to adult male Fisher F344 rats. One to 30 days after exposure, lung and brain tissue were collected and processed for laser scanning microscopy (LSM). Paraffin sections from the lung were immune-labelled for the specific antigen presenting cell markers CD68 (macrophrages) and MHC class II (dendritic cells), sections for the brain were labeled with NeuN (neurons). Stained sections were qualitatively analyzed using LSM. Furthermore, the 
expression of pro-inflammatory cytokines was measured in the lung tissue using ELISA.

Preliminary data showed that the vast majority of NP localized in the lung parenchyma were found inside alveolar macrophages, whereas NP were rarely associated with dendritic cells. No NP were seen in the controlateral right control lung. The cytokine profile in the BALF showed an enhanced secretion of most proinflammatory cytokines (IL-5, IL-6, IFN-gamma, IL-1beta, IL-10) until 30d after exposure. In the brain tissue PVA-SPIONS were associated with, mostly localized intra-cellularly.

Conclusion: Based on the preliminary data we conclude that, though a considerable amount of PVA-SPIONS are primarily phagocytosed by macrophages, but enter the circulation and cross the blood-brain barrier. Furthermore, the delivered particles induced an inflammatory response in the lung as assessed by cytokine patterns. More research is required to elucidate precise translocation mechanisms of biomedical NP delivered to the respiratory tract and their downstream fate. Moreover, a in-depth assessment of particle-lung immune cell interaction is a prerequisite to develop novel nanocarrier strategies for drugs and vaccines via the pulmonary route.

\section{P83}

\section{Sequential Resection of Superior Vena Cava to} Decrease Cerebral Complication During T4 Bronchial or Thymic Carcinoma Surgery

P. Morand ${ }^{1}$, F. Beckers' ${ }^{2}$, C. Ludwig ${ }^{2}$, E. Stoelben ${ }^{2}$

${ }^{1}$ Inselspital, Bern, ${ }^{2}$ Lungenklinik Köln Merheim, Köln, D

Background: For advanced bronchial or thymic tumors infiltrating the superior vena cava (SVC) it is indicated to perform a resection of the SVC. Usually the SVC is completely clamped, resected and then bypassed. During clamping, intracerebral blood pressure increases and can cause brain damage. We describe here an alternative technique of resection of the SVC in order to reduce the risk of cerebral injury.
Methods: 10 patients ( 7 men, 3 women), mean age: 57,4 years (44-69 year). Seven patients suffered from central bronchial carcinoma and 3 patients had a thymic carcinoma. 1.To establish a bypass between the left vena anonyma and the right atrium using an $8 \mathrm{~mm}$ Gore-Tex graft consolidated with rings. 2. Complete resection of the SVC and the tumor. 3. To establish a bypass between the right vena anonyma and the right atrium, distally to the vena cava stump, using an $8 \mathrm{~mm}$ Gore-Tex graft consolidated with rings. Prophylactic post-operative anticoagulation with low weight heparin twice a day is indicated.

Results: 1 patient because of a thrombosis had to be reoperated. None of the patients presented cerebral injury or bleeding.

Conclusion: Sequential resection of the SVC is technically feasible and secure. This technique is a good alternative to reduce cerebral complications associated with complete resection of the SVC when total clamping is used.

\section{P84 \\ Intermediate Term Outcome of Lung Resection in High Risk Patients with Non Small Cell Lung Cancer}

T. Schneider, M. Kuhn, M. Furrer

Cantonal Hospital of Graubünden, Chur

Background: Complete anatomical resection of the primary tumor remains the treatment of choice for patients with stage I-IIIa Non Small Cell Lung Cancer (NSCLC) and preoperative pulmonary evaluation is usually done to define high risk patients. We were interested in the intermediate term outcome of patients not having fulfilled all cut-off values of the preoperative functional assessment.

Methods: In 34 patients with NSCLC all treated in our institution in 2010 the onco-surgical intention of treatment was an anatomical resection. To asses the functional operability the criteria of Basel (BC) were used\#. 8 patients (24\%) didn't fulfil completely the citeria and were operated nevertheless. ICU and hospital stay, 30-day-mortality and 6-min walk-distance-test 30 days postoperatively were individually analysed in these patients.

Table 1. (for Abstract P84)

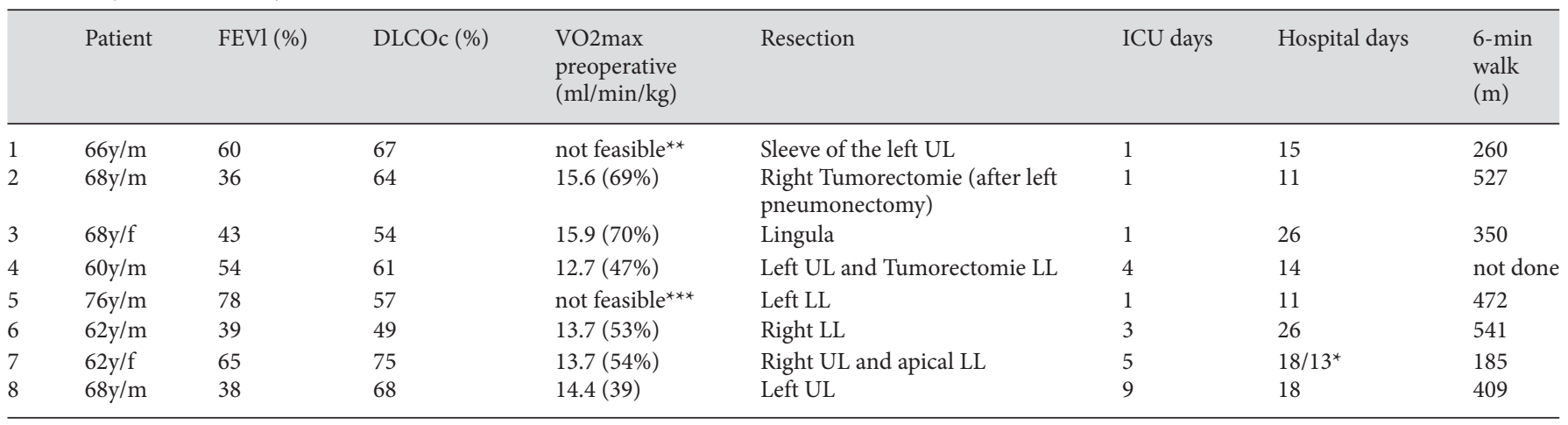

${ }^{*}$ acute arteriel occlusion/severe gastric bleeding; ${ }^{* *}$ severe gonarthritis; ${ }^{* * *}$ general weakness after induction chemotherapy. 
Results: In four cases the calculated postoperative VO2max was below the critical threshold of $40 \%(\geq 10 \mathrm{ml} / \mathrm{min} / \mathrm{kg}$ ) (pat. 4 , $6-8$ ). In the remaining two cases (pat 2-3) the absolute value of FEV1 was about $1000 \mathrm{ml}$. The 30 -day-mortality was $0 \%$. All results are summarized in the table below.

Conclusions: $24 \%$ of our lung cancer patients operated in 2010 were classified as critical for a surgical treatment. Even not having fulfilled completely the criteria for functional resectability the 30 -day-mortality was $0 \%$ and the intermediate outcome was acceptable in 7 out of 8 high risk patients.

\#Eur Resp J 1998; 11:198-212/modified in Eur Resp J 2009; 33:1206-1215

\section{P85 \\ Seasonal Influenza Vaccination 2010/2011: Uptake and Reasons for Non-Vaccination Among Lung Transplant Recipients}

M. Schuurmans, E. Perret, A. Zuercher, G. Fretz, A. Boehler

University Hospital of Zurich, Zurich

Background: Seasonal influenza vaccination is recommended for lung transplant recipients (LTR) in order to prevent complications of infection such as acute allograft rejection. Yearly vaccination is recommended by the attending physicians and covered by the health care insurance. Vaccination is voluntary. Systematic assessments of influenza vaccination uptake among LTR are not available. The aim of this study is to assess vaccination uptake and reasons for non-vaccination among LTR.

Methods: All LTR were offered to be vaccinated during followup visits on a voluntary basis. Transient contraindications (signs of infection) lead commonly to postponement of vaccination. Patients without contraindications not volunteering for vaccination were asked to fill in a widely used questionnaire assessing reasons for non-vaccination.

Results: Of 168 eligible LTR 135 (80\%) were vaccinated in a 4 -month period. The most frequent reasons for vaccination refusal were in descending order: (1) Belief, that the vaccination is not sufficiently effective, (2) belief, that one is unlikely to be affected by influenza disease, (3) recurring infections not permitting vaccination, (4) hospitalisation for complications, and (5) increased prednisone dose making vaccination response unlikely.

Conclusions: Vaccination uptake among LTR was high in comparison to published data for other solid organ transplant recipients. Reasons for non-vaccination were partly due to beliefs related vaccination effectiveness. A considerable number of LTR were not vaccinated despite lack of clear contraindications. Improving knowledge about vaccine effectiveness among LTR may lead to improved seasonal influenza vaccination uptake among these LTR.

\section{P86 \\ Pandemic H1N1 Influenza Virus Vaccination in Lung Transplant Recipients: Coverage, Safety and Clinical Effectiveness in the Zurich Cohort}

M. Schuurmans, G.M. Tini, L. Dalar, G. Fretz, C. Benden, A. Boehler

University Hospital of Zurich, Zurich

Background: Pandemic 2009 H1N1 influenza virus (H1N1) infection is considered harmful to lung transplant recipients (LTR). Vaccination against this virus is recommended for LTR, but little is known about associated benefits and risks. The aim is to document safety and clinical effectiveness of the $\mathrm{H} 1 \mathrm{~N} 1$ vaccine in LTR.

Methods: All LTR received an information letter on the H1N1 pandemic, hygiene and vaccination recommendations. After completing a questionnaire, volunteering LTR received Pandemrix * (H1N1 2009 Monovalent AS03-Adjuvanted Vaccine, GSK). Adverse events (AEs) were documented at short-term follow-up visits and by telephone. Any flu-like symptoms were reported and a low threshold for performing nasal/pharyngeal swabs for virus detection was maintained. Evidence for allograft rejection was systematically assessed during 6 months post-vaccination.

Results: Of 168 eligible LTR (107 were already vaccinated for 2009 seasonal influenza) 148 LTR (88\%) received at least one vaccination with the $\mathrm{H} 1 \mathrm{~N} 1$ vaccine and 115 received a second dose. After the first vaccination, $44 \%$ had no adverse reactions. Six selflimiting severe AEs occurred, the remainder were minor to moderate predominantly injection site reactions. After the second vaccination, AEs were clearly less frequent. All AEs resolved completely. No evidence for an increased rate of allograft rejection was noted among vaccinated LTR. Documented H1N1 infection occurred in 2 of 148 vaccinated LTR in contrast to 5 infections in 20 non-vaccinated LTR.

Conclusions: $\mathrm{H} 1 \mathrm{~N} 1$ vaccination was generally well tolerated with mild to moderate predominantly local AEs in most LTR and few self-limiting severe events. Clinical effectiveness was good.

\section{Poster Session 3 Sleep/Mechanical Ventilation}

\section{P87 \\ Impact of Age on Respiratory Polygraphy Quality in Children}

A. Wavre Oberhänsli, J. Haba-Rubio, R. Heinzer

CHUV, Lausanne

Introduction: Obstructive sleep apnea syndrome (OSAS) is a condition encountered in about $3 \%$ of the children. Polysomnography is the gold standard diagnostic procedure but 
Table 1. (for Abstract P88)

\begin{tabular}{|c|c|c|c|}
\hline & $490 \mathrm{~m}$ & $1630 \mathrm{~m}$ & $2590 \mathrm{~m}$ \\
\hline Nocturnal oxygen saturation, \% & $95+/-1$ & $93+/-2^{*}$ & $89+/-2 * \#$ \\
\hline AHI total, $1 / \mathrm{h}$ & $9.2+-7.5$ & $12.7+/-8.9^{*}$ & $23.7+/-18.3^{* \#}$ \\
\hline AHI obstructive, $1 / \mathrm{h}$ & $6.1+/-7.0$ & $4.7+4.4$ & $6.1+/-6.1$ \\
\hline AHI central, 1/h & $3.1+/-4.0$ & $8.0+/-8.5^{\star}$ & $17.6+/-17.1$ *\# \\
\hline median CPAP, mbar & $8.2+/-2.2$ & $8.9+/-2.5^{*}$ & $9.7+/-2.7 * \#$ \\
\hline 90.\%ile CPAP, mbar & $10.8+/-2.6$ & $11.6+/-2.7^{\star}$ & $12.2+/-2.4^{\star \#}$ \\
\hline Slow wave sleep, \% & $14.6+/-7.8$ & $13.8+/-8.6$ & $10.7+/-6.3^{\star \#}$ \\
\hline Driving simulator reaction time, $\mathrm{s}$ & $2.4+/-0.7$ & $2.3+/-0.8$ & $2.3+/-0.8$ \\
\hline Subjective insomnia & $1(0 ; 2)$ & $1(0 ; 1)$ & $1(0 ; 2)$ \\
\hline
\end{tabular}

${ }^{\star} \mathrm{P}<0.05$ vs. $490 \mathrm{~m}$; ${ }^{\#} \mathrm{P}<0.05$ vs. $1630 \mathrm{~m}$. ${ }^{4} 4$ point scale $0=$ slept as usual to $3=$ could not sleep at all. Means (SD) with exception of medians, quartiles for subjective sleep quality.

limited channel home recording (respiratory polygraphy, RP) is increasingly used to detect OSA in children. Compared to polysomnography, $\mathrm{RP}$ is a cheaper and more convenient procedure but data on its' reliability in children are scarce. The aim of our study was to determine the impact of age on RP recording quality.

Methods: RP recordings of children ( 1 to 17 years of age) referred for suspected OSAS were retrospectively analyzed. Quality of the recordings was assessed based on the percentage of reliable signal obtained on the 3 following channels: nasal pressure, oxygen saturation ( $\mathrm{SaO} 2)$, and thorax or abdominal bands. The presence of reliable signals in these 3 channels during at least $50 \%$ of the recording was the minimal requirement to consider the test reliable.

Results: RP recordings of 55 children (40 male) were analyzed. Mean age was 6.7 y.o. (range 16 months-17 years+ 5 months). Mean recording time was $9.38+/-0.9$ hours. Mean Apneahypopnea index (AHI) was $5.2 /$ h. $32.7 \%$ of the children had an $\mathrm{AHI}<1 / \mathrm{h}, 25.4 \%$ had an AHI between 1 and $5 / \mathrm{h}$ and $21.8 \%$ had an $\mathrm{AHI}>5 / \mathrm{h}$. Eleven recordings (20\%) did not meet the minimal quality requirements. There was a statistically significant correlation between children's age and the proportion of the recording time with reliable signals $(\mathrm{r}=0.317 ; \mathrm{p}=0.018) .76 \%$ of the recordings in children above 6 years of age met the minimal quality criteria, whereas only $38 \%$ met these criteria in children under 6 y.o. (Fisher exact test. $\mathrm{p}=0.006$ ).

Conclusion: PG recordings quality is correlated with children's age and significantly improves above 6 years of age.

\section{P88 \\ Does AutoCPAP Therapy Control Breathing Disturbances in Patients with Obstructive Sleep Apnoea Syndrome at Altitude?}

T.D. Latshang, R.M. Henn, Y. Nussbaumer-Ochsner, S. Ulrich Somaini, M. Kohler, K.E. Bloch

University Hospital of Zurich, Zurich

Background: We have previously reported that lowlanders with obstructive sleep apnea syndrome (OSA) experience pronounced breathing disturbances during sleep due to frequent central apnea and impaired driving simulator performance when staying at altitude (Thorax 2010;165:429). The optimal treatment in this setting is unknown. We investigated whether computer-controlled CPAP (autoCPAP) adequately controls breathing disturbances in OSA patients during an altitude sojourn.

Methods: 49 OSA patients living at $<600 \mathrm{~m}$ on long-term CPAP participated in a cross-over trial. Mean age \pm SD was $61 \pm 9 \mathrm{y}$, apnea/hypopnea index (AHI) off CPAP at $490 \mathrm{~m}$ was $46 \pm 22 / \mathrm{h}$. Patients were studied at 3 altitudes for 1 night each in random order while being treated with autoCPAP (5-15mbar): Zurich, $490 \mathrm{~m}$, and Davos at $1630 \mathrm{~m}$ and at $2590 \mathrm{~m}$, respectively. Polysomnography, driving simulator and questionnaire evaluations were performed at the 3 locations.

Results: Pressure delivered and measured by CPAP machines at the 3 altitudes was accurate within $1 \mathrm{mbar}$ in comparison to a water manometer. The obstructive AHI remained unchanged but central AHI and computer adjusted mask pressure increased with increasing altitude (table).

Conclusion: In OSA patients at moderate altitude, autoCPAP adequately controlled obstructive apnea/hypopnea although central events were not completely prevented. A moderate increase in total AHI and a minor reduction in deep sleep at altitude did not impair driving simulator reaction time and subjective sleep quality. Therefore, autoCPAP appears to be suitable for OSA therapy during a short term altitude sojourn.

Support: Swiss National Science Foundation, Lungenliga Zurich and Schaffhausen, Switzerland. 
P89

\section{The Effect of Simulated Obstructive Apnoea and Hypopnoea on Aortic Diameter and Blood Pressure}

A.-C. Stöwhas ${ }^{1}$, M. Namdar ${ }^{1}$, P. Biaggi ${ }^{1}$, E.W. Russi ${ }^{1}$, K.E. Bloch ${ }^{1}$, J.R. Stradling ${ }^{2}$, M. Kohler ${ }^{1}$

${ }^{1}$ University Hospital of Zurich, Zurich, ${ }^{2}$ Churchill Hospital, Oxford, UK

Background: Preliminary evidence supports an association between obstructive sleep apnea (OSA) and thoracic aortic dilatation. The mechanisms through which OSA may promote thoracic aortic dilatation are incompletely understood. Therefore, we studied the acute effects of simulated apnea and hypopnea on aortic diameter and blood pressure in humans.

Methods: In 20 healthy volunteers the diameter of the aortic root was measured by echocardiography and peripheral blood pressure was continuously recorded prior, during and immediately after simulated obstructive hypopnea (inspiration through threshold load), simulated obstructive apnea (Mueller maneuver), endexpiratory central apnea and normal breathing in randomized order.

Results: Proximal aortic diameter increased significantly during inspiration through a threshold load $(+6.48 \%$ (SE3.03), $\mathrm{p}=0.007)$, but not during Mueller maneuver $(+3.86 \%$ (SE2.71), $\mathrm{p}=0.336)$ or end-expiratory central apnea (+0.62\% (SE 2.94), $\mathrm{p}=0.445)$. Maneuver-induced changes in mean blood pressure were observed during inspiration through a threshold load $(-10.5$ (SE2.2) $\mathrm{mmHg}, \mathrm{p}<0.001$ ), the Mueller maneuver $(-8.8$ (SE2.4) $\mathrm{mmHg}, \mathrm{p}<0.001)$, and end-expiratory central apnea $(-4.2$ (SE1.4) $\mathrm{mmHg}, \mathrm{p}=0.052)$. There was a significant increase in mean blood pressure on release of threshold load inspiration (+8.1 (SE2.9) $\mathrm{mmHg}, \mathrm{p}=0.002)$, Mueller maneuver $(+10.7$ (SE2.9) $\mathrm{mmHg}$, $\mathrm{p}<0.001)$ and end-expiratory central apnea $(+10.6(\mathrm{SE} 2.5) \mathrm{mmHg}$, $\mathrm{p}<0.001)$.

Conclusions: Simulated obstructive hypopnea/apnea and central apnea induced considerable changes in blood pressure and obstructive hypopnea was associated with an increase in proximal aortic diameter. Further studies are needed to investigate effects of apnea and hypopnea on transmural aortic pressure and aortic diameter in order to define the role of OSA in the pathogenesis of aortic dilatation.

\section{P90 \\ CPAP Therapy Withdrawal - A Model to Evaluate Treatments for Obstructive Sleep Apnoea}

A.-C. Stoewhas ${ }^{1}$, K.E. Bloch' ${ }^{1}$, E.W. Russi ${ }^{1}$, J.R. Stradling ${ }^{2}$, M. Kohler ${ }^{1}$

${ }^{1}$ University Hospital Zurich, Zurich, ${ }^{2}$ Churchill Hospital, Oxford, UK

Rationale: Evaluating new treatments for OSA recruiting previously untreated patients in randomised controlled trials is time consuming and expensive. A more efficient model to investigate OSA treatments could be during withdrawal of CPAP.
Objectives: To determine the effects of two weeks CPAP withdrawal on sleep-disordered breathing, sleepiness and psychomotor performance.

Methods: 41 OSA patients on CPAP were randomized to either withdraw CPAP (subtherapeutic-CPAP) or continue CPAP for 14 days. At baseline, 7 and 14 days, apnea/hypopnea index (AHI,1/h), oxygen desaturation index (ODI,1/h), Epworth sleepiness scale (ESS), Osler test, divided attention driving simulator (DADS) and psychomotor vigilance test (PVT) were assessed.

Measurements and main Results: Withdrawal of CPAP increased $\mathrm{AHI}$ at 7 and 14 days (mean difference in AHI change +31.9 (95\%CI 20.1,43.7) and +33.5 (95\%CI 22.4/44.6), respectively) and ODI (mean difference in ODI change +26.3 (95\%CI $16.6,36.0)$ and +26.4 (95\%CI 16.1,36.8), respectively) in comparison to continuation of CPAP ( $\mathrm{p}<0.001$ for all comparisons). ESS increased significantly at 7 and 14 days in the CPAP withdrawal group compared to the CPAP group (mean difference in ESS change $+1.9(95 \%$ CI $0.4,3.3)$ and +2.7 (95\%CI 1.2,4.3), $\mathrm{p}=0.015$ and $\mathrm{p}<0.001$, respectively). Withdrawal of CPAP was not associated with deterioration in the Osler test, DADS and the PVT at 7 and 14 days.

Conclusions: CPAP withdrawal usually leads to a rapid recurrence of OSA and a slow return of subjective sleepiness, but is not associated with deterioration in psychomotor performance within 14 days. Therefore, this model seems suitable and safe to evaluate treatment effects on OSA.

\section{P91 \\ Berlin and STOP-BANG Questionnaires for Detecting Sleep Apnoea in the General Population}

D. Andries, J. Haba-Rubio, F. Bastardot, N. Tobback, P. Vollenweider, M. Tafti, R. Heinzer

CHUV, Lausanne

Introduction: Berlin questionnaire (BQ) has been proposed as a screening tool for identifying patients at risk for obstructive sleep apnea (OSA). STOP-BANG score (Snoring, Tiredness during daytime, Observed apnea, High blood Pressure, Body mass index $>35$, Age $>50$, Neck circumference $>40 \mathrm{~cm}$, Gender) has been shown to be a useful tool to screen for obstructive sleep apnea (OSA) during preoperative evaluation. The aim of our study is to evaluate the performance of these questionnaires for detecting OSA in a large sample of middle-aged general population.

Methods: 458 subjects ( $47.7 \%$ women, $50.6 \pm 7.5$ years old, BMI $25.2 \pm 4.9 \mathrm{~kg} / \mathrm{m}^{2}$ ) participating in an ongoing populationbased cohort study (HypnoLaus, Lausanne, Switzerland) underwent a complete polysomnographic recording at home and an extensive clinical workup including STOP BANG parameters and BQ questionnaire. BQ includes 3 categories: 1) witnessed apnea and snoring 2) daytime sleepiness and 3) obesity or hypertension. A positive score in 2 or more categories was considered suggestive of OSA. A STOP-Bang score of 3 or more out of a possible 8 was considered suggestive of OSA. These scores were compared to apnea-hypopnea index (AHI) determined by PSG. AHI was scored according to 2007 AASM criteria. 


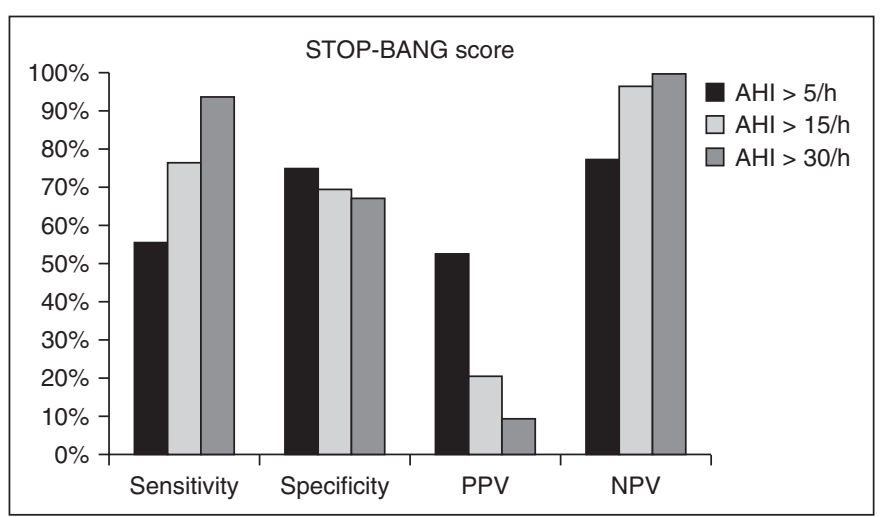

Fig. 1.

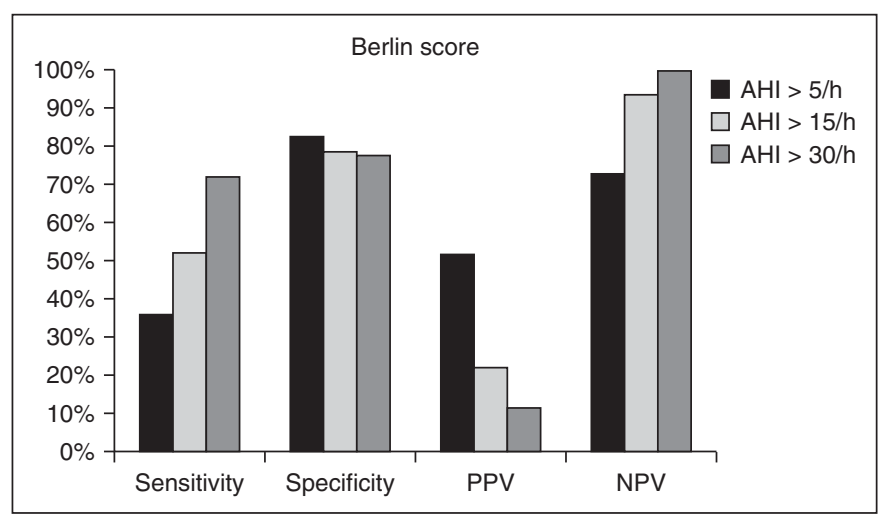

Fig. 2.

Results: Prevalence of positive BQ score was $24.4 \%$. BQ sensitivity, specificity, positive predictive value (PPV) and negative predictive value (NPV) to detect OSA were $36.1 \%, 82.4 \%, 51.8 \%$ and $72.9 \%$ respectively for an $\mathrm{AHI}>5 / \mathrm{h} ; 52.1 \%, 78.8 \%, 21.9 \%$ and 93.5\% for an $\mathrm{AHI}>15$; and $72.2 \%, 77.6 \%, 11.4 \%$ and $98.6 \%$ for an AHI $>30 / h$. Mean STOP-BANG score was $2.16 \pm 1.37$. 34.9\% of the subjects had a score $\geq 3$. To detect OSA with AHI thresholds of 5/h, 15/h and 30/h, sensitivity, specificity, PPV and NPV were respectively $55.6 \%, 75.2 \%, 52.5 \%$ and $77.5 \%$ for an $\mathrm{AHI}>5 / \mathrm{h}$; 76.7\%, 69.4\%, 20.6\% and $96.6 \%$ for an AHI $>15 / \mathrm{h} ; 93.8 \%, 67.2 \%$, $9.4 \%$ and $99.7 \%$ for an $\mathrm{AHI}>30 / \mathrm{h}$. For an $\mathrm{AHI}>5 / \mathrm{h}$, the area under the ROC curve was 0.60 for BQ score and 0.67 for STOP-BANG score.

Conclusion: Due to their poor sensitivity, BQ questionnaire and STOP-BANG score do not seem to be adequate screening tools for OSA in the general population. They could however be useful in ruling out severe OSA considering their high negative predictive value for an $\mathrm{AHI}>30 / \mathrm{h}$.
P92

Mathematical Processing of Airflow Signal Can Identify Loss of Respiratory Rate Variability as a Marker of Patient-ventilator Asynchrony in NonInvasive Ventilation

D. Adler ${ }^{1}$, R. Grave de Peralta Menendez', F. Espa ${ }^{1}$, J.-L. Pépin², S. Perrig ${ }^{1}$, J.-P. Janssens ${ }^{1}$

${ }^{1} \mathrm{HUG}$, Genève, ${ }^{2} \mathrm{CHU}$, Grenoble, F

Background: Patient-ventilator asynchrony caused by wasted efforts (PVA-WE) is frequent in COPD patients undergoing noninvasive ventilation (NIV) who are considered effectively treated. PVA-WE remains undetected if only simple monitoring tools such as pulsoximetry and transcutaneous capnography (TcPCO2) are used. Polysomnography (PSG) under NIV, the gold standard for NIV monitoring, is expensive and not always available. Indeed, development of cheap monitoring tools is urgently needed. Here we show that simple mathematical processing of airflow signal alone can provide information on PVA-WE unavailable so far.

Methods: Severe COPD patients under NIV were studied on two consecutive nights. PSG under NIV was recorded with the patient's usual ventilator settings (night1). Ventilator settings were adjusted using on-line PSG with TcPCO2/SaO2 monitoring in order to reduce PVA-WE (night2). Patients were compared according to their back-up respiratory rate ('low': $n=3$; RR: 13-14; 'high': n=3: RR: 18-19). Off line mathematical analysis of the flow signal (OMAF) was done using homemade Matlab code in two steps. 1) Semi automatic (supervised) segmentation to detect breath cycles main points and 2) Automatic computation of RR interval with its graphical representation. OMAF results were compared in a 10 minutes window with PVA-WE (night1) and a 10 minutes window without PVA-WE obtained after adjustment of NIV settings (night2). Simultaneous analysis of $\%$ of spontaneous inspirations was obtained from ventilator built-in software. Variability (or its absence) is demonstrated by the temporal course of RR interval over time.

Results (figure 1): PVA-WE occurred in all patients (panel a). In patients with a 'low' back-up rate, PVA-WE was associated with a loss of respiratory rate variability (panel b) and a reduction in percentage of spontaneous inspirations (25-52\%) on the first night. Adjusting NIV settings with PSG on the second night decreased PVA-WE in all patients. In the 'low back-up rate' group, it increased respiratory rate variability (panel c) and percentage of spontaneous inspirations (85-93\%) wich may reflect an improved ability to trigger the ventilator.

Conclusion: Mathematical processing of flow signal to assess PVA-WE is technically feasible in clinical practice. Loss of variability and low $\%$ of spontaneous inspiration as surrogate markers for PVA-WE require further investigation since they occurred only in 'low' back-up rate COPD patients. 


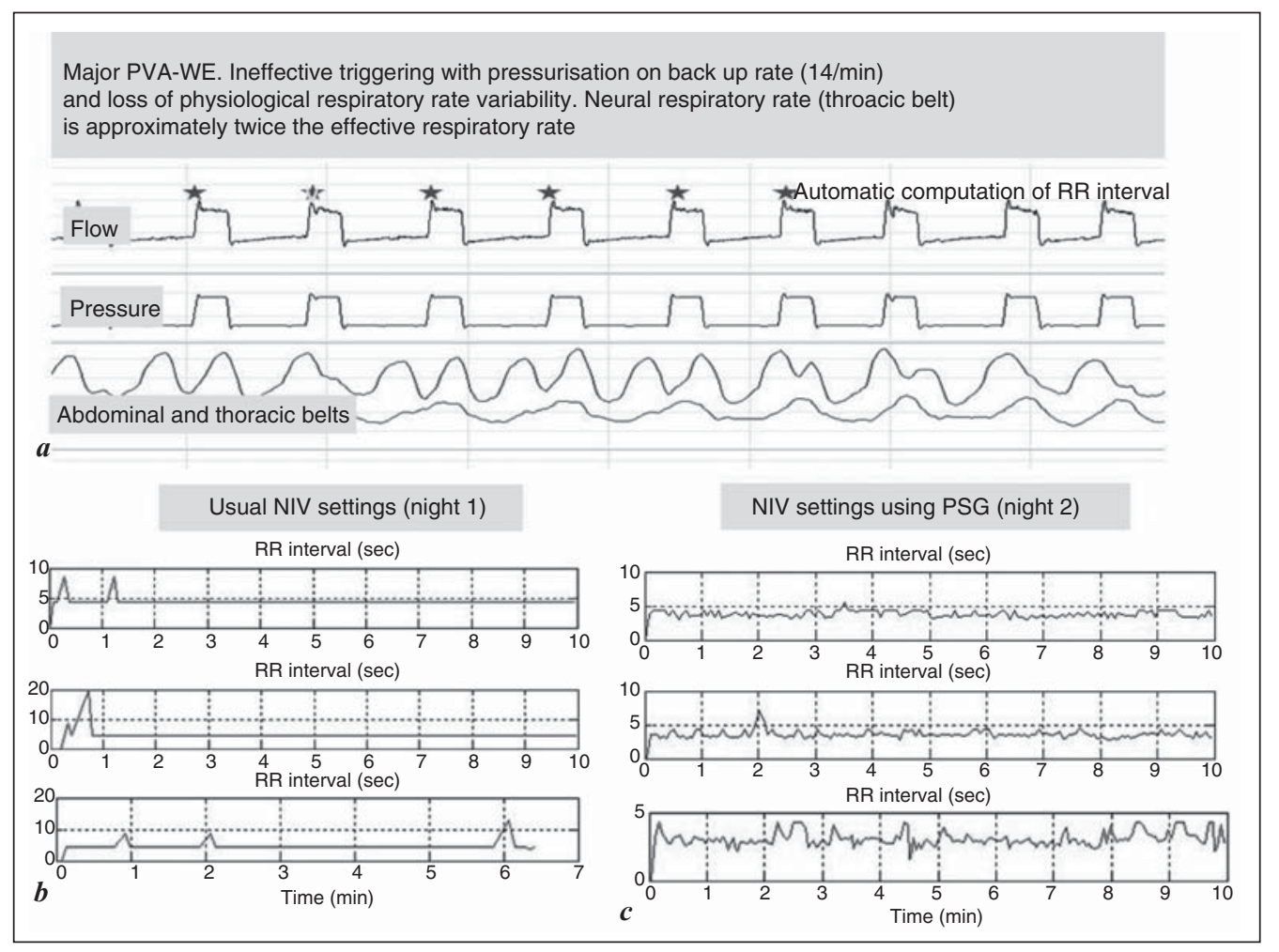

Fig. 1. (for Abstract P92)

\section{P93 \\ Severe Metabolic Acidosis in Chronically Ventilated Duchenne Muscular Dystrophy Patients}

C.M. Lo Cascio, T.D. Latshang, M. Kohler, T. Fehr, K.E. Bloch

University Hospital of Zurich, Zurich

Background: Duchenne muscular dystrophy (DMD) leads to progressive muscular weakness, loss of ambulation, tetraparesis and chronic respiratory failure around age 9, 14 and 20, respectively. Long-term mechanical ventilation improves quality of life and has increased the life expectancy to currently 35 years. With prolongation of life previously unrecognized DMD complications might arise. The purpose of this ongoing study is to analyze clinical characteristics and causes of severe metabolic acidosis, a condition we have recently observed in several DMD patients.

Methods: DMD patients admitted to the emergency room, university hospital of Zurich, with severe metabolic acidosis during the last 5 years were included. All were on long-term positive pressure ventilation, 6 out of 8 were ventilated for $24 \mathrm{~h} /$ day. History, physical examination, blood and urinary chemistry and arterial blood gas analysis were evaluated.

Results: Eight DMD patients $(26 \pm 4$ y) were included. At admission, they suffered from dyspnea despite assisted ventilation by mask $(n=7)$ or tracheostomy $(n=1)$ and from abdominal discomfort and distension. All were treated for chronic constipation with laxatives and other measures, in 5 of 8 this treatment had been intensified in the days before admission. All patients had reduced their food and fluid intake in the last $1-5$ days; 2 patients presented with cough and purulent sputum. Patients were in severely reduced general condition with tachycardia (median 130, range 96-156/min) although mean arterial blood pressure was not reduced (median 93, range $75-103 \mathrm{mmHg}$ ). Body temperature was $36.5-40.0^{\circ} \mathrm{C}$. The table shows results of the arterial blood gas analysis at admission on assisted ventilation and supplemental oxygen titrated to maintain SpO2 $>90 \%$. Urinary ketones were qualitatively positive in all. Rehydration, parenteral and/or enteral nutrition, pharmacological regulation of intestinal motility and antibiotics lead to complete recovery in all patients.

Table 1. Results of arterial blood gas analysis

\begin{tabular}{lllllrr}
\hline Variable & $\mathrm{pH}$ & $\mathrm{PaCO} 2, \mathrm{kPa}$ & $\mathrm{HCO} 3-, \mathrm{mmol} / \mathrm{l}$ & Lactate, $\mathrm{mmol} / \mathrm{l}$ & $\mathrm{K}+, \mathrm{mmol} / \mathrm{l}$ & Anion gap, mmol/l \\
\hline Normal range & $7.35-7.45$ & $4.6-6.0$ & $22-26$ & $0.7-2.1$ & $3.3-4.5$ & $8.0-12.0$ \\
Mean $\pm \mathrm{SD}$ & $7.1 \pm 0.1$ & $4.02 \pm 1.30$ & $11.2 \pm 5.1$ & $0.8 \pm 0.2$ & $3.5 \pm 0.5$ & $20.1 \pm 8.0$ \\
\hline
\end{tabular}


Conclusions: Our observations in older DMD patients on longterm ventilation demonstrate that severe, metabolic acidosis related to chronic constipation and its treatment, reduced fluid and food intake and associated respiratory infection may result in a life-threatening condition. Thus, adequate regulation of bowel movements, hydration and nutrition seem to be crucial in prevention of this complication.

Grant support: Lunge Zürich

\section{P94 \\ Home-Based versus Hospital-Based Monitoring of Long-Term Home Ventilation: A Pilot Study}

\section{P. Pasquina, P. Farr, P. Bourqui, P.-O. Bridevaux, J.-P. Janssens}

University Hospital of Geneva, Geneva

Background: Many centers monitor patients under home ventilation (HMV) by short hospitalizations, for at least $24 \mathrm{hrs}$. This allows performing tests such as nocturnal oxymetry, capnography, polygraphy, arterial blood gases (ABG), pulmonary function tests (PFT), checking interface, and data provided by ventilator software. However, for many patients, these hospital stays may appear as tedious and unnecessary; furthermore, because of the large number of HMV patients, obtaining beds for HMV patients is a problem; conversely, a substantial amount of information can be collected at home and may suffice for monitoring HMV patients; also home-based evaluation may be less expensive.

Method: To compare a standard 24-hour hospital based evaluation vs. a home-based evaluation performed by specialized nurses with a subsequent outpatient medical control. Home-based evaluation includes items of medical history, vital signs, quantification of lower limb edema, spirometry, $\mathrm{SpO} 2$, nocturnal oximetry, home ABG, and downloading on a PC of data provided by ventilator software (compliance, leaks, tidal volume and minute ventilation, apnea and hypopnea index). Two successive 1 year periods were compared ('Usual care' vs 'Home based evaluation') in stable patients. Endpoints were Health-Related Quality of Life (HRQL: SF-36 and SGRQ), patient satisfaction (17 item questionnaire), costs, and hospitalizations or emergency visits for acute cardiopulmonary symptoms.

Results: Fifteen patients (aged 65 \pm 15 yrs, under HMV for $6.1 \pm 3.2$ yrs) with restrictive disorders (CVF: $66 \pm 27 \%$ predicted) were included. Two patients died of cardio-pulmonary failure during the initial part of study. For all other patients, PFT, ABG, nocturnal oximetry, compliance showed no significant change between hospital and home evaluation. HRQL questionnaires (SF36 and SGRQ) remained unchanged, while patients noted improvements in several items of satisfaction questionnaire and globally preferred home-based evaluation. Interestingly, home evaluation induced lesser direct and indirect costs for patients than hospital evaluations. Conversely, going to the hospital was considered as inconvenient for $60 \%$ of all patients. No acute hospitalization occurred during the 'home-based' observation period.

Conclusions: For stable patients under HMV, coupling a homebased thorough evaluation by specialized nurses with an outpatient medical visit is feasible, increases patient satisfaction and may decrease cost of medical follow-up in selected patients.

\section{Poster Session 4 Rehabilitation}

\author{
P95 \\ Pulmonary Rehabilitation in Switzerland 2007/2008 \\ M. Frey' ${ }^{1}$ P. Zuehlke², U. Lagler ${ }^{3}$, W. Karrer ${ }^{4}$ \\ ${ }^{1}$ Klinik Barmelweid, Barmelweid, '2Lungenliga Schweiz, Bern, \\ ${ }^{3}$ Pneumologische Praxis/Privatklinik Bethanien, Zürich, \\ ${ }^{4}$ Luzerner Höhenklinik Montana, Crans Montana and Working \\ Group (WG) Pulmonary Rehabilitation and Patient Education of \\ the Swiss Respiratory Society
}

Introduction: In 2002 a WG of the ALVR (Arbeitsgemeinschaft Leistungserbringer-Versicherer für wirtschaftliche und qualitätsgerechte Rehabilitation) have published quality criteria for pulmonary rehabilitation programs (PR). Since January 2005, Swiss health insurance companies must pay for PR in in- and outpatients on a legal basis, but no agreement could be achieved on the rate to be paid for out-patient rehabilitation. To accredit a program, audits must be performed by two specialists. The audit focuses on the quality of the program structure and the rehabilitation process, as well as on outcome measurements. Currently 10 in- and 55 out-patient programs are accredited.

Methods: To assure the quality of the programs, an assessment is done every two years by means of a questionnaire, assessing the number of patients treated, the diagnoses and the complications. In 2005/2006 the questionnaire was sent out for the first time. Actually all programs programs returned the questionnaire. Failure to reply on two consecutive questionnaires results in loss of accreditation.

Results: In 2007 and 2008 respectively, 4882/5075 patients in an in-patient and 1232/1354 patients in an out-patient have been treated. $87 \%$ of the program directors replied (05/06: 77\%). As in 2005/2006 most patients suffered from COPD (46\%/48\%), but also patients with other diagnoses qualifying for PR were enrolled in the programs. Out-patient programs mainly treat COPD patients (68\% in 2007 and 2008). Acute exacerbations occurred in $154 / 155$, fatal complications in $24 / 14$ cases. About $92 \%$ of the patients completed the programs. However, the dropout rate is considerably higher in the out-patient (19\%/15\%) programs than in the in-patient programs $(4 \% / 4 \%)$.

Conclusions: The number of treated patients remained at a constant level. In the same period there was an increase in outpatient programs. The response rate was also higher in the second period.

Switzerland has a widespread network of PR programs (in- and out-patient settings), which is still expanding. Over the past years many of the accredited programs also have improved in quality, such as infrastructure and rehabilitation process.

The questionnaire is a good tool to maintain and improve the program quality in collaboration with the institutions. 


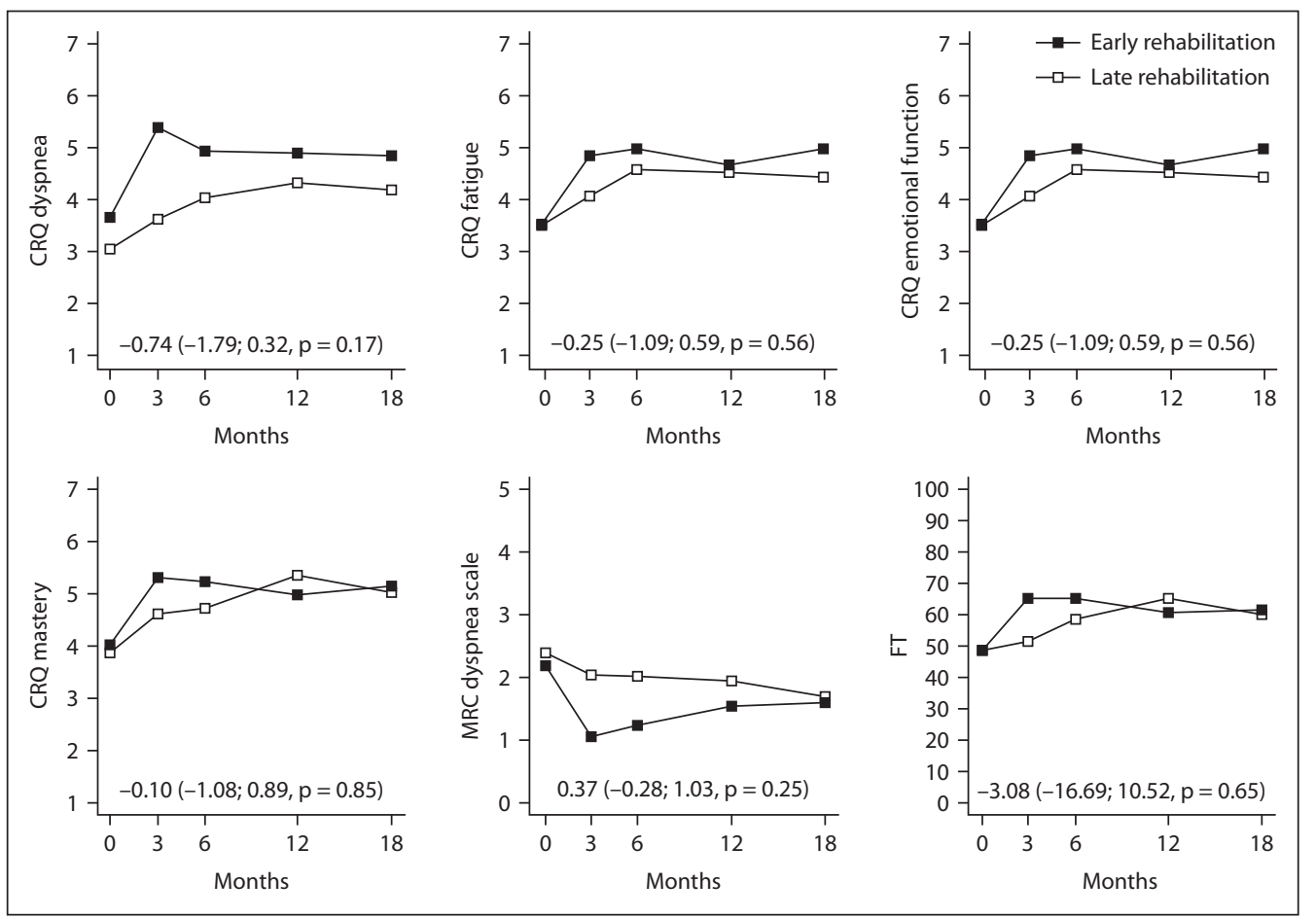

Fig.1. (for Abstract P96)

\section{P96 \\ Timing of Pulmonary Rehabilitation - Swiss Trial on Pulmonary Rehabilitation after COPD Exacerbation (SOPRE)}

M. Puhan ${ }^{1}$, A. Spaar ${ }^{1}$, M. Frey ${ }^{2}$, A. Turk ${ }^{3}$, O. Brändli ${ }^{3}$, D. Ritscher ${ }^{4}$,

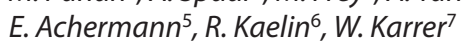

${ }^{1}$ University of Zurich, Zurich, ${ }^{2}$ Klinik Barmelweid, Barmelweid, ${ }^{3}$ Zürcher Höhenklinik Wald, Wald-Faltigberg, ${ }^{4}$ Lungenpraxis Morgental, Zurich, ${ }^{5}$ Limmattalspital, Schlieren, ${ }^{6}$ Private Practice, Morges, ${ }^{7}$ Luzerner Höhenklinik Montana, Crans-Montana

Background: While in some countries most patients are referred to (late) pulmonary rehabilitation in stable state, patients are traditionally referred to (early) pulmonary rehabilitation just after an exacerbation in countries such as Switzerland. There are no trials comparing different timings of referral to pulmonary rehabilitation. Our aim was to compare the effects of early and late pulmonary rehabilitation on exacerbation rates and health-related quality of life (HRQL) in exacerbated COPD patients.

Methods: We randomized COPD patients (GOLD stages II-IV) and a recent exacerbation to early (within two weeks) or to late pulmonary rehabilitation (starting six months after randomization and in stable state). The primary outcome was the exacerbation rate over 18 months and secondary outcomes included HRQL and mortality. We used multivariate analyses and an intention-totreat analysis approach.

Results: We randomized 36 patients. Patients with early rehabilitation $(n=19)$ had, on average, 2.61 (SD 2.96) exacerbations requiring systemic corticosteroids and/or antibiotics compared to 2.77 (SD 3.41) in patients with late rehabilitation (adjusted incidence rate ratio 0.83 [95\% CI $0.43-1.63$ ], $\mathrm{p}=0.60$ ). Over the 18 months period, Chronic Respiratory Questionnaire domain scores were higher for patients with early rehabilitation ( 0.54 for dyspnea, 0.76 for fatigue, 0.71 for emotional function and 0.25 for mastery) but differences did not reach statistical significance (Figure).

Conclusion: Exacerbation rates were similar but our trial indicates that early rehabilitation may lead to faster recovery from exacerbations and may provide an effective post-exacerbation management for COPD patients.

Trial registration: ISRCTN8461231

P97

The Effects of Controlled Breathing During Pulmonary Rehabilitation in Patients with COPD

A.J.R. van Gestel' ${ }^{1}$, M. Kohler ${ }^{1}$, J. Steier ${ }^{2}$, S. Teschler ${ }^{3}$, E.W. Russi ${ }^{1}$, H. Teschler ${ }^{3}$

${ }^{1}$ University Hospital of Zurich, Zurich, ${ }^{2}$ Kings College London School of Medicine, London, UK, ${ }^{3}$ University Hospital Ruhrlandklinik, Essen, D

Background: Conventional pulmonary rehabilitation programs improve exercise tolerance but have no effect on pulmonary function in patients with COPD. The role of controlled breathing using 
respiratory biofeedback during rehabilitation of patients with COPD remains unclear.

Objectives: To compare the effects of a conventional 4-week pulmonary rehabilitation program with those of rehabilitation plus controlled breathing interventions.

Methods: A randomized controlled trial was performed. Pulmonary function (FEV1), exercise capacity (6-minute walking distance, 6MWD), health-related quality of life (chronic respiratory questionnaire, CRQ) and cardiac autonomic function (rMSSD) were evaluated.

Results: Forty COPD patients (mean \pm SD age $66.1 \pm 6.4$, FEV1 $45.9 \pm 17.4 \%$ predicted $)$ were randomized to rehabilitation $(n=20)$ or rehabilitation plus controlled breathing $(n=20)$. There were no statistically significant differences between the two groups regarding the change in FEV 1 (mean difference $-0.8 \%$ predicted, 95\% CI -4.4 to $2.9 \%$ predicted, $\mathrm{p}=0.33$ ), $6 \mathrm{MWD}$ (mean difference $12.2 \mathrm{~m}$, $95 \% \mathrm{CI}-37.4$ to $12.2 \mathrm{~m}, \mathrm{p}=0.16$ ), CRQ (mean difference in total score $0.2,95 \% \mathrm{CI}-0.1$ to $0.4, \mathrm{p}=0.11$ ) and rMSSD (mean difference $2.2 \mathrm{~ms}, 95 \% \mathrm{CI}-20.8$ to $25.1 \mathrm{~ms}, \mathrm{p}=0.51$ ).

Conclusions: In patients with COPD undergoing a pulmonary rehabilitation program, controlled breathing using respiratory biofeedback has no effect on exercise capacity, pulmonary function, quality of life or cardiac autonomic function.

Key words: Controlled breathing, chronic obstructive pulmonary disease, diaphragmatic breathing, pursed lips breathing, pulmonary rehabilitation, respiratory biofeedback

\section{P98 \\ Changes in the CRQ, CAT and Updated BODE Index by Pulmonary Rehabilitation}

\section{A. Stucki, Y. van der Feijst}

Berner Reha Zentrum, Heiligenschwendi

Background: There is strong evidence showing that pulmonary rehabilitation programs are beneficial to patients with COPD in order to improve exercise capacity, symptoms, and health-related quality of life. The responsiveness and the changes in health related quality of life scores, e.g. CRQ scores are well known. It is unclear to which extend a standardised in-hospital rehabilitation program may change the short and simple new COPD assessment (CAT) test score and the updated BODE Index in severe and very-severe COPD patients.

Method: In a prospective study we assessed the CRQ, CAT and updated BODE scores in severe and very-severe COPD patients (GOLD class III and IV) before and after attending a multidisciplinary in-hospital pulmonary rehabilitation program. The assessment comprised in addition sociodemographic and health data: age, sex, co-morbidities, medication, use of oxygen and or CPAP or BIPAP. Patients followed an inpatient respiratory rehabilitation of approximately 3 week's duration. The rehabilitation program included interval exercise or continuous exercise, breathing therapy, relaxation therapy, nutritional therapy, guidedwalking tours, and patient education.

According to the initial BODE Index and due to statistical reasons we formed two BODE severity groups; group one with $0-7$ points and group two with $8-15$ points.
Results: 315 patients attending a pulmonary rehabilitation program were assessd between first of July and end of December 2010. Thereof 132 patients presented with severe or very-severe COPD. The mean age was 69.2 years, exactly one third female, mean duration of stay 19.7 days.

I. Both groups showed a significant improvement for all four CRQ domains. Only for the domain dyspnea the improvement was significant smaller for patients with a higher BODE score (second group).

II. The CAT score showed a similar significant improvement in both groups (reduction of 4.5 and 5 points respectively)

III. Also the updated BODE Index showed a significant reduction in both groups, however, this reduction was significant less for the second group.

Conclusion: The simple CAT test and the updated Bode Index might both provide a valid assessment for changes in an inpatient pulmonary rehabilitation program for severe and very-severe COPD patients.

\section{P99}

Patient Needs and Expectations Regarding Development of Self-help Groups for Rare Pulmonary Diseases

L. Kolopp', G. Neuhaus' , J.-M. Egger' ${ }^{2}$, I. Progin'², N. Schawalder', R. Holderegger ${ }^{3}$, R. Lazor ${ }^{4}$

${ }^{1}$ University of Lausanne, Lausanne, ${ }^{2}$ Ligue Pulmonaire Suisse, Berne, ${ }^{3}$ University Hospital of Berne, Berne, ${ }^{4} \mathrm{CHUV}$, Lausanne

Patients with rare diseases often suffer from isolation and lack of information about their disorder, due to its rarity. Self-help groups may help affected individuals to cope with their illness, but such groups do not exist for many rare disorders, and individual patients may lack skills, motivation or resources to build such groups themselves. We surveyed patients affected by rare pulmonary diseases to determine their needs and expectations about contacts with other persons affected by the same disease and selfhelp groups. Physicians of the Swiss Respiratory Society were requested to forward a questionnaire to their patients affected by a rare disease. Data were collected by the Swiss Pulmonary League. 86 filled questionnaires were available. The diseases were sarcoidosis $(52 \%)$, interstitial lung disease $(9 \%)$, primary ciliary dyskinesia $(8 \%)$, pulmonary vasculitis $(7 \%)$, cystic lung diseases $(7 \%)$, alpha-1-antitrypsine deficiency (6\%), and others (11\%). Patients expressed the following interests: meet other persons affected by a rare pulmonary disease $(27 \%)$, meet other persons affected by the same disease (68\%), participate to a self-help group without supervision by a specialist (64\%), participate to a self-help group with supervision by a specialist (71\%), have direct individual contact with persons affected by the same disease (60\%), provide their address to the Swiss Pulmonary League and allow to be contacted by other persons having the same disease (76\%), participate to an internet forum on their disease (53\%), and be recorded in a rare disease registry (91\%). $73 \%$ reported they had found understandable information about their disease, $43 \%$ knew about an existing self-help group, and $33 \%$ have been in contact with that group. Only 16\% could imagine to found a self-help group themselves. 
Conclusion: the majority of surveyed patients were interested by contacts with other persons having the same disease. Internet forum were found less interesting than personal contact with individuals or groups. Only a small proportion would engage in the development of a self-help group. External support and guidance might be useful to assist patients with rare diseases in the development of self-help groups.

\section{P100 \\ Correlation of Physical Performance and Quality of Life in Adult Patients with Cystic Fibrosis}

M. Hofer, B. Fiechter Lienert, T. Kurowski, A. Boehler

University Hospital of Zurich, Zurich

Aim: To prospectively evaluate physical performance and quality of life in a cohort of adult CF patients (non transplant candidates).

Methods: Physical performance and quality of life were measured with pulmonary function (FEV1), six-minute walk test (6MWT), SF-36 and St George's Respiratory Questionnaire (SGRQ). Symptoms of depression where evaluated with the short form of the Patient Health Questionnaire (PHQ-D).

Results: From 62 patients 23 patients ( 10 females) with a mean age of $31 \pm 9 y$ performed the investigation. Mean body mass index (BMI) was $22 \pm 3 \mathrm{~kg} / \mathrm{m} 2$, FEV1 $2.3 \pm 1.3 \mathrm{~L}(59 \pm 26 \%)$ and $6 \mathrm{MWT}$ $726 \pm 128 \mathrm{~m}$. Values of the 8 domains of SF36 were $86 \pm 17$ (physical functioning, normal healthy adults $>57$ ), $86 \pm 29$ (physical role, $>56$ ), $88 \pm 16$ (bodily pain, >62), $57 \pm 16$ (general health, >45), $59 \pm 12$ (vitality, >47), $86 \pm 16$ (social functioning, $>57$ ), $84 \pm 28$ (emotional role, $>55$ ) and $78 \pm 9$ (mental health, $>41$ ). Total score of SGRQ was $23 \pm 14$ (healthy adults $<6$ ), with a symptom score of $45 \pm 20(<12)$, activity score of $24 \pm 22(<2)$ and impact score of $15 \pm 13(<2)$. In the short PHQ-D no patients had signs of a major depression or anxiety.

We found a significant correlation of FEV1, FEV1\%pred and $6 \mathrm{MWT} \%$ pred with the SF-36 domain 'physical functioning' and all domains of the SGRQ ( $r>0.5$ and $\mathrm{p}<0.01)$.

Conclusion: In our adult CF patients we found normal values for the 8 domains of the SF36. On the other hand, the more specific respiratory questionnaire (SGRQ) showed an impairment of respiration related quality of life compared to healthy adults. There is a significant correlation between physical functioning (FEV1 and 6MWT) and respiratory symptoms.
P101

\section{Physical Performance in Adult Cystic Fibrosis Patients: A Longitudinal Survey}

\section{B. Fiechter Lienert, T. Kurowski, A. Boehler, M. Hofer}

University Hospital, Zurich, Zurich

Aim: To evaluate the physical performance status including muscle strength and endurance in a cohort of adult cystic fibrosis patients.

Methods: Assessments were performed at baseline and at 6 months including pulmonary function (FEV1), six-minutes walking test (6MWT), Kasch Pulse Recovery Step Test (KPRST, scoring 0-4) and maximum voluntary elbow/knee flexion and extension strength (EF,EE, KF,KE).

Results: From 62 patients of the adult CF unit 23 patients (10 females) with a mean age of $31 \pm 9$ y performed the first investigation. Mean body mass index (BMI) was $22 \pm 3 \mathrm{~kg} / \mathrm{m} 2$, FEV1 $2.3 \pm 1.3 \mathrm{~L}(59 \pm 26 \%$ predicted), $6 \mathrm{MWT} 726 \pm 128 \mathrm{~m}$, EF $22 \pm 7$ $(42 \pm 24 \%$ percentile $)$, EE $15 \pm 6(55 \pm 27 \%)$, KF $27 \pm 9(57 \pm 24 \%)$ and $\mathrm{KE} 36 \pm 12(31 \pm 17 \%)$. EF, EE, KF and $\mathrm{KE}$ was in $16,8,8$ and 18 patients below 50 percentile, respectively. Mean score of KPRST was $2.1 \pm 1.2$ with 3 patients with poor, 3 fair, 9 average, 5 good and 3 excellent performance. At the end of the $6 \mathrm{MWT}$ mean pulse rose up to $41 \pm 16$, and $17(74 \%)$ patients had a desaturation of $-5.4 \pm 4.1 \%$ below baseline saturation. There was no significant correlation of desaturation and FEV1. In 14 patients follow-up visits were performed. There were no significant changes in BMI, FEV1, 6MWT, KPRST and elbow and knee extension/flexion strength.

We found a significant correlation between FEV1\%pred and $6 \mathrm{MWT} \%$ pred $(\mathrm{R}=0.79, \mathrm{p}<0.0001)$. There was a weak correlation of FEV1\%pred and 6MWT\%pred with elbow and knee extension and flexion ( $\mathrm{R} 0.45-0.7, \mathrm{p}<0.01)$.

Conclusion: Desaturation in $6 \mathrm{MWT}$ is frequent in our adult $\mathrm{CF}$ and independent of FEV1. KPRST is often impaired. 6MWT and FEV1 were significantly correlated but no correlation was found with KPRST. Elbow and knee flexion and extension strength was impaired with a weak correlation with FEV1 and 6MWT but not KPRST. 


\section{Poster Session 5 Interstitial Lung Disease}

\section{P102 \\ Pulmonary Hypertension in Sarcoidosis - A Case of Successful Treatment with Immunsuppressive Therapy}

\section{A. Kunz, J. Pichler Hefti, T. Geiser, S.R. Ott}

Inselspital, Bern

The guidelines for management of pulmonary hypertension (PH) recommend a diagnostic work-up of newly diagnosed $\mathrm{PH}$ to search for underlying diseases, before affirming the diagnosis of idiopathic pulmonary artery hypertension (PAH) and initiation of treatment. This is of major importance because treatment of the underlying disease can significantly improve $\mathrm{PH}$ and should, hence, be started before considering $\mathrm{PH}$ - specific treatment. We report the case of a patient with sarcoidosis associated $\mathrm{PH}$ who improved significantly after initiation of immunosuppressive treatment with steroids. A 41 years old male reported dyspnea during mountaineering caused by recurrent high altitude edema (HAPE). The right heart catheter revealed an increased pulmonary pressure of sPAP $66 \mathrm{mmHg}$ with an exercise induced increase to sPAP $110 \mathrm{mmHg}$. At that time, a pulmonary or cardiac diseases, HIV infection, vasculits or chronic pulmonary embolism was excluded. A mediastinal lymphadenopathy was interpreted $\mathrm{PH}$ associated because TBNA nor lung biopsy showed findings suggestive for sarcoidosis. Hence, PAH was diagnosed and treatment with bosentan was initiated leading to decrease of pulmonary pressure and resolution of clinical symptoms. Two years later, the patient deteriorated, HAPE reoccurred and pulmonary pressure increased to SPAP $70 \mathrm{mmHg}$. Our reassessment including thoracic CT-scan showed progression of mediastinal lymphadenopathy and EBUS-TBNA revealed epitheloid cell granulomas confirming the diagnosis of sarcoidosis. Immunosuppressive treatment (steroids followed by azathioprin) was started; $\mathrm{PH}$ significantly decreased to sPAP $45 \mathrm{mmHg}$ and the patient was free of clinical symptoms, even after dose reduction of bosentan. Our case is specific for two reasons. 1) To our knowledge, this is the first report of HAPE as first manifestation of sarcoidosis associated PH (SAPH); 2) It shows the need for careful exclusion of underlying diseases in $\mathrm{PH}$, because specific treatment can improve $\mathrm{PH}$. SAPE is a common $(5-74 \%)$ and serious complication of sarcoidosis that is accompanied by a 10 -fold increased mortality as compared to sarcoidosis without $\mathrm{PH}$. In sarcoidosis, $\mathrm{PH}$ is usually mild to moderate, but is associated with a poor prognosis (5-year survival 59\%). Causative treatment of underlying sarcoidosis can improve $\mathrm{PH}$ without PH specific therapy. Thus, all newly diagnosed cases of $\mathrm{PH}$ should be evaluated for underlying diseases, before initiation of $\mathrm{PH}$ specific treatement.

\section{P103 \\ Treatment of Pulmonary Sarcoidosis with Mycophenolate Mofetil - An Effective Steroid- Sparing Agent?}

A.-K. Brill, T. Herren, T. Geiser

University Hospital of Berne, Berne

Background: In chronic pulmonary sarcoidosis, stabilisation of lung function and symptomatic relief often requires long-term treatment with systemic corticosteroids. To reduce steroid burden, immunosuppressive agents are added to the therapeutic regimen. We aimed to examine the efficiency of mycophenolate mofetil $(\mathrm{MMF})$ as a steroid-sparing agent.

Methods: In this single centre observational trial, we retrospectively analyzed ten patients with biopsy-proven pulmonary sarcoidosis of radiological stage II $(n=4)$, stage III $(n=1)$, and stage IV $(n=5)$ treated with MMF and systemic corticosteroids within the period between June 2004 and December 2010. Data on corticosteroid dose and duration of MMF administration were documented and clinical course classified by analyzing repeated pulmonary function tests and dyspnea levels. Mild (i.e., continuation of MMF) and severe adverse effects (i.e., interruption of MMF) were recorded.

Results: In $9 / 10$ patients (90\%), who had been on prior immunosuppressive therapies including corticosteroids and steroidsparing agents (azathioprine, $\mathrm{n}=7$; ciclosporine, $\mathrm{n}=1$; azathioprine and pentoxifylline, $\mathrm{n}=1$ ), MMF was introduced due to side effects or unsatisfactory response. In 1/10 patients (10\%) MMF was used as first line steroid-sparing agent. Mean MMF dose varied between $1722 \pm 440 \mathrm{mg}$ and $1425 \pm 567 \mathrm{mg} / 24 \mathrm{~h}$ to maintain plasma through levels between $1-3 \mathrm{mg} / \mathrm{L}$. Corticosteroid dose was significantly $(\mathrm{p}<0.001$, general linear model repeated measures) reduced (7.9 $\pm 2.8 \mathrm{mg}$ and $6.9 \pm 2.5 \mathrm{mg}$ of prednisolone at 6 and 12 months, respectively), while pulmonary function and dyspnea levels were stable in $8 / 10(80 \%)$ or improved in $2 / 10$ patients $(20 \%)$. Daily doses of prednisolone were $<=7.5 \mathrm{mg}$ in $77 \%$ and $<=10 \mathrm{mg}$ in $97 \%$ regarding the total time of follow-up (315 months). Mild adverse effects (i.e, mild leukopenia (> $1.5 \mathrm{G} / \mathrm{l})$, self-limiting respiratory tract infection; gastrointestinal symptoms, moderate increase of liver enzymes) were documented in $7 / 10$ patients (70\%); severe adverse effects (i.e., recurrent respiratory tract infections requiring antibiotic therapy) were encountered in $1 / 10$ patients $(10 \%)$ leading to the discontinuation of MMF after 18 months.

Conclusion: Our results indicate that MMF allows to reduce corticosteroid dosage in chronic pulmonary sarcoidosis treatment to levels below $10 \mathrm{mg} /$ day in most of the patients paralleled by stable or improved clinical condition, suggesting a steroid-sparing effect of MMF in pulmonary sarcoidosis. 


\section{P104}

A Rare Cause of Severe Airway Obstruction: Laryngeal Involvement in Sarcoidosis

\author{
A.-K. Brill' ', P. Ludwig' ', M. Hagemann' ', P. Dumont ${ }^{2}$, T. Geiser ${ }^{1}$, \\ S.R. Ott ${ }^{1}$ \\ ${ }^{1}$ Universitiy Hospital of Berne, Berne, ${ }^{2}$ Cantonal Hospital of \\ Fribourg, Fribourg
}

Background: Laryngeal involvement in sarcoidosis is rare (incidence $0.5-1.4 \%$ ) and often diagnosed with delay and already severely obstructed upper airways in symptomatic patients. Surgical airway management is often unavoidable. We present a case of successful conservative management.

Case report: A 47-year old Ethiopian with biopsy-proven facial cutaneous, endonasal and suspected laryngeal sarcoidosis since 2008 presented with progressive dyspnea on exertion, dry cough and pre-existent hoarseness. Treatment was limited to application of cutaneous topical steroids. Clinically, a biphasic wheezing was present. Pulmonary function testing showed marked limitations of in- and expiratory flow rates consistent with a fixed upper airway obstruction (Fig. 1 A). Normal lung volumes and diffusion capacity were present. Cervical and thoracic scans showed typical bihilary lymphadenopathy and few areas with subpleural ground glass opacities. In addition to cervical lymphadenopathy there was a circular glottic and supraglottic narrowing of the upper airways. Flexible fiberoptic nasolaryngoscopy revealed a granuloma on the posterior pharyngeal wall and complete paralysis of the scarred appearing left vocal cord, fixed in paramedian position. There was pale edematous mucosal tissue with granulomas predominantly on the left arytenoids and aryepiglottic folds. The width of the remaining dorsal glottis was $5 \mathrm{~mm}$. Complete closure of the vocal cords was still possible. Search for other systemic manifestations did not reveal any cardiac or ophthalmic involvement.

Treatment and course: An immunosuppressive therapy with high dose systemic prednisolone (starting dose $1 \mathrm{mg} / \mathrm{kg}$ body weight) was initiated. Azathioprine was added as steroid-sparing agent after tapering prednisolone to $20 \mathrm{mg}$ /day. This resulted in complete remission of stridor and dyspnea paralleled by a marked improvement of respiratory flow rates (Fig 1 B). Surgical or invasive therapy could be avoided. Two month after initiation of therapy a naso-palatine fistula developed in a pre-existing grauloma. An oral device was prescribed.

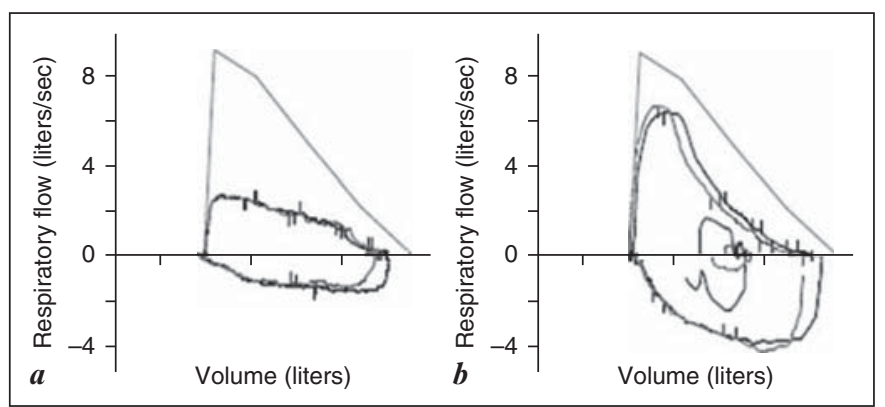

Fig. 1.
Conclusion: Laryngeal involvement in sarcoidosis can cause debilitating symptoms and a possibly life threatening airway obstruction. Thus, in patients with sarcoidosis and progressive dyspnea laryngeal involvement should be considered in the differential diagnosis as early diagnosis and treatment can prevent tracheotomia.

\section{P105}

\section{Multiple Pulmonary Nodules in a Smoker - Got It?}

L. Kern, J. Rüdiger, C. Meyenberger, R. Rodriguez, M.H. Brutsche Cantonal Hospital St. Gallen, St. Gallen

Introduction: The incidental detection of multiple pulmonary nodules in a smoking patient is highly suspicious for the presence of malignant disease of either pulmonary or metastatic origin. However, pulmonary nodules can also be seen in non-malignant extrapulmonary diseases including inflammatory bowel disease.

Case presentation: A 46-year old patient was referred after the detection of multiple pulmonary nodules in both lower lung lobes in an abdominal computed tomography (CT) scan performed for the investigation of frequent loose stools (Figure 1). The patient had a smoking history of 20 pack years and was otherwise healthy. $\mathrm{He}$ complained of temperatures of around $37.2^{\circ}$ Celsius, night sweats and weakness. Symptoms started six weeks prior to admission and did not respond to antibiotics. Upon presentation, the patient was alert and in no distress. Abdominal examination and chest auscultation were unremarkable. Positron emission tomography (PET) scan showed an elevated uptake with a standardized uptake value (SUV) of 4.6.

Pulmonary function tests and arterial blood gas analysis were normal.

Bronchoscopy with brushings was non-diagnostic. Bronchoalveolar lavage (BAL) revealed normal total- and differential cell counts. A transthoracic biopsy showed foreign-body type granuloma and intraalveolar fibroblasts, consistent with organizing pneumonia (Figure 2).

Colonoscopy revealed pancolitis without terminal ileitis. Biopsies of the colonic mucosa showed changes compatible with ulcerative colitis (UC).

The diagnosis of organizing pneumonia (OP) in the context of UC was retained. A therapy with mesalazin was started and the patient's bowel symptoms resolved rapidly. However, he still complained of subfebrile temperatures, weakness and night sweats. Even though no functional impairment induced by OP was found, a course of systemic steroids was started. One week later, the patient was free of symptoms.

Discussion: UC is an inflammatory bowel disease usually limited to the colon mucosa. However, extraintestinal manifestations are well described and include also lung disease. Airway involvement is mostly seen and often presents as chronic cough, mucoid impaction, bronchial obstruction and bronchiectasis. OP in the context of UC has been described before in a few case reports, but seems to be a rare entity with the last publication dating back to 2003. Furthermore, pulmonary manifestations of UC usually appear years after diagnosis, unlike in this case. 

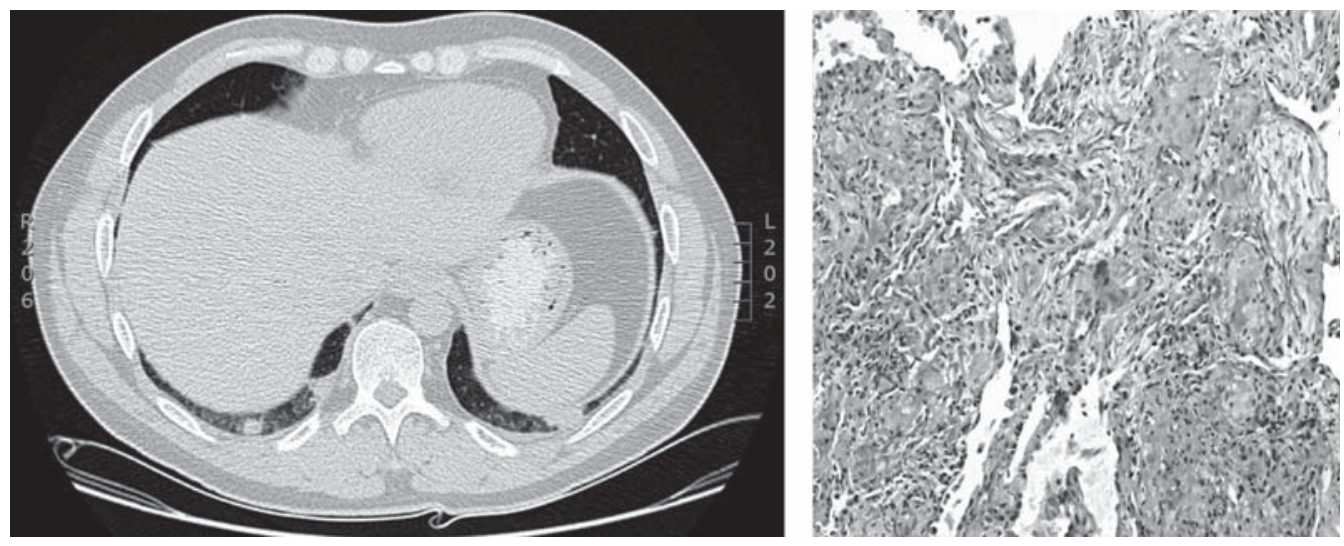

Fig. 1, 2. (for Abstract P105)

\section{P106 \\ Shrinking Lung Syndrome Successfully Treated with Cyclophosphamide and Rituximab}

E. Langenskiold ' , A. Bonetti ${ }^{2}$, J.W. Fitting ${ }^{1}$, R. Heinzer ${ }^{1}$, J. Dudler ${ }^{3}$, F. Spertini ${ }^{1}$, R. Lazor ${ }^{1}$

${ }^{1} \mathrm{CHUV}$, Lausanne, ${ }^{2}$ Private Practice, Lausanne, ${ }^{3} \mathrm{HFR}$, Fribourg

Shrinking lung syndrome (SLS) is a rare manifestation of systemic lupus erythematosus (SLE). Its pathogenesis remains controversial and the treatment is not well defined. We report the case of a 28-year old woman, who was diagnosed with SLE-Sjögren ovelap syndrome in February 2009. She had invalidating joint pain, for which she received prednisone up to $30 \mathrm{mg} /$ day, hydroxychloroquine, and successively methotrexate and azathioprine, with no clinical benefit but a weight gain of $24 \mathrm{~kg}$. In March 2009, she was admitted to ICU for acute pulmonary embolism, with transient right heart failure. No antiphospholipid antibodies were detected. In April, lung function tests were normal, except DLCO at $66 \%$ pred. In July, she developed within a few days NYHA III dyspnea, pleuritic chest pain, dry cough, and severe orthopnea. Respiratory function tests showed the appearance of severe restrictive ventilatory defect, with $50 \%$ volume loss compared to April. Review of consecutive chest X-rays demonstrated progressive elevation of both hemi diaphragms. Chest computed tomography showed neither recurrence of pulmonary embolism, nor pleural or interstitial lung disease, and echocardiography ruled out significant pulmonary hypertension and pericardial effusion. Maximal inspiratory and expiratory pressures were slightly lowered and supine vital capacity (VC) showed 700ml drop (-35\%) compared to sitting VC. Electromagnetic phrenic nerve stimulation confirmed diaphragm paralysis. SLS was diagnosed. In addition to long acting beta2agonists and theophylline, cyclophosphamide $600 \mathrm{mg} / \mathrm{m} 2$ every 4 weeks and rituximab $2 \mathrm{x} 1 \mathrm{~g}$ every 6 month were respectively introduced for respiratory and joint involvements. After 7 perfusions of cyclophosphamide (stopped because of side effects) and $2 \times 2$ doses of rituximab, her condition significantly improved: dyspnea decreased to NYHA stage II, pleuritic pain almost disappeared, and lung volumes increased ( $\mathrm{VC}+70 \%$ compared to baseline). Cyclophosphamide was replaced by azathioprine, rituximab is being continued, and prednisone has been tapered to $7.5 \mathrm{mg} /$ day. Although the relative value of cyclophosphamide and rituximab cannot be determined, this drug combination was effective for treatment of SLS in this case. A favourable effect of rituximab alone has been reported in only two cases. This drug deserves further study in this indication.

\section{P107 \\ Pulmonary Granulomatosis and Organising Pneumonia Induced by Ipilimumab}

L. Noirez, G. Berthod, I. Letovanec, J. Mazza Stalder, O. Michielin,
R. Lazor

CHUV, Lausanne

Background: Cytotoxic T lymphocyte antigen 4 (CTLA-4) is expressed on the surface of activated T-cells and mediates downregulation of immune responses to allow tolerance of self antigens. Ipilimumab, a human monoclonal antibody blocking CTLA-4, has been recently shown effective in advanced melanoma, presumably through enhanced T-cell immune response against tumor self antigens. However, severe immune-related adverse events (IRAEs) have been observed after ipilimumab, including dermatitis, enteritis, and hepatitis.

Methods: We report the case of a 63 year-old man who presented with sarcoid-like pulmonary granulomatosis and organising pneumonia after ipilimumab therapy for melanoma.

Results: The patient received of Ipilimumab $3 \mathrm{mg} / \mathrm{kg}$ (270mg) for advanced melanoma metastatic in particular to the lungs and mediastinal lymph nodes, and unresponsive to conventional chemotherapy by DTIC (dacarbazine, $1000 \mathrm{mg} / \mathrm{m} 2 / 3$ weeks). 4 weeks after the last dose, he developed nonproductive cough and progressive NHYA stage II then III dyspnea. Bilateral crackles were heard at chest auscultation. The chest CT-scan showed regression of pulmonary and mediastinal metastases, but the appearance of new multiple subpleural and parenchymal nodular opacities. Lung functions tests showed moderate restrictive ventilatory defect and 
reduction of carbon monoxide diffusion capacity. Bronchoalveolar lavage showed lymphocytic alveolitis (58\%) with increased CD4/ CD8 ratio. Bacteriology, including acid-fast bacilli and $M$. tuberculosis PCR, was negative. Surgical lung biopsy showed numerous giant-cell granulomas frequently exhibiting central necrosis with predominantly interlobular and subpleural distribution, as well as moderate organising pneumonia pattern with mild interstitial inflammation and fibrosis. No infectious agent was identified. Considering the close temporal relationship between ipilimumab treatment and onset of lung disease, a pulmonary IRAE of ipilimumab was diagnosed. Oral prednisone was started. After 3 weeks, respiratory symptoms were reduced, lung function tests and chest CT scan were moderately improved.

Conclusion: Ipilimumab can induce severe pulmonary toxicity characterised by the unusual combination of sarcoid-like granulomatosis and organising pneumonia, two disorders associated with $\mathrm{T}$ cell-mediated responses. We hypothesize that the enhanced T-cell immunity induced by ipilimumab played a key role in their pathogenesis.

\section{P108 \\ When Bone Invades the Lung}

M. Prella, D. Sistek, A. Pasche, S. Rotman, L.P. Nicod, J.-D. Aubert

CHUV, Lausanne

Background: Pulmonary alveolar microlithiasis(PAM) is a rare lung disease characterized by intra-alveolar formation of calciumphosphate microliths. Most patients remain symptom-free for many years until the development of respiratory failure and death.

Methods: We report a successful case of bilateral lung transplantation in a patient with PAM complicated by pulmonary hypertension $(\mathrm{PH})$.

Results: A 55-year-old non-smoker woman with PAM was referred for lung transplantation. PAM was found incidentally at the age of 22 years because of characteristic radiological findings. The patient was asymptomatic until the age of 47 years when she presented with progressive dyspnea leading to oxygen dependency. Pulmonary function tests showed a restrictive ventilatory defect with total lung capacity of $58 \%$ of predicted (2.3L) and diffuse capacity for CO of $1.5 \mathrm{mmol} / \mathrm{kPA}$.min (23\% of predicted). The CT-scan showed numerous calcified nodules with extensive conglomerations and subpleural calcifications. An echocardiogram revealed $\mathrm{PH}$ with right ventricular dilatation and dysfunction. Right heart catheterization confirmed pre-capillary $\mathrm{PH}$ with mean pulmonary arterial pressure (PAP) of $40 \mathrm{mmHg}$ and a cardiac index of $1.7 \mathrm{l} / \mathrm{min} / \mathrm{m}^{2}$. The patient underwent bilateral lung transplantation after 10 months on the waiting list. Cardiopulmonary bypass was necessary due to hemodynamic instability. Histological examination confirmed PAM with multiple foci of metaplastic ossification together with extramedullary hematopoieses. In the early postoperative period the patient presented with hemorrhagic shock requiring surgical haemostasis. 10 months after transplantation, the patient is free of dyspnea without the need for supplemental oxygen. An echocardiogram shows normal PAP and right ventricular function.
Conclusion: Lung transplantation is the only effective treatment for end-stage PAM. Recurrence after transplantation has not been reported to date. The timing of transplantation is not fully established as the course of the disease is indolent and there are no established prognostic indices. Postoperative hemorrhage is the most frequent complication, especially in patients with advanced pleural involvement and needing cardiopulmonary by-pass. Patients should be referred to a transplantation centre before the development of right ventricular dysfunction.

\section{Poster Session 6 Infectious Disease}

\section{P109 \\ Use of a PCR-based Mycobacterium Tuberculosis Strain Genotyping to Systematically Analyse all Isolates Collected at Geneva University Hospital During 2009 and 2010}

\author{
B. Ninet, C. Ionati, S. Sauthier, J. Schrenzel, J.-P. Janssens \\ University Hospital Geneva, Geneva
}

Background: Genotyping analysis of Mycobacterium tuberculosis isolates is increasingly applied in the control of tuberculosis outbreaks. During 2009 and 2010, we tested in our laboratory an easier and faster method than the gold standard RFLP or other PCR-based methods such as spoligotyping or mycobacterial intersped repetitive unit-variable-number tandem repeat (MIRUVNTR) typing.

Methods: The diversiLabTM strain typing M.tuberculosis kit (Biomérieux), a commercially available repetitive sequence-based PCR method, was used to characterize 96 strains isolated from patients newly diagnosed with tuberculosis during 2009 and 2010 in Geneva.

Results: Among all patients, only two small clusters of three patients each emerged. The first cluster consisted of a married couple and another woman with no evident epidemiological link but of the same ethnic origin. The second cluster included two twin infants and their aunt who looked after them. Fourteen other groups, including two patients with no obvious link, were detected. All these strains displayed few bands and were sometimes very close to each other (with a homology greater than 99\%).

Conclusion: The diversiLab Mycobacterium tuberculosis strain typing can detect transmission between infected cases but remains difficult to interpret with some isolates, most likely because of suboptimal discrimination capabilities. A significant amount of DNA is necessary to perform this technique which is sometimes difficult to obtain with mycobacterium strains. Nevertheless, repPCR for mycobacteria can be a first method of discrimination. Epidemiologic link must be confirmed by another typing method in case of identical result between patients. 


\section{P110 \\ High Rate of Completion of Preventive Therapy for Latent Tuberculosis Infection Among Asylum Seekers. A Pilot Study in Vaud County}

A. Sarivalasis ${ }^{1}$, E. Langenskiold ${ }^{2}$, C. Lutchmaya-Flick ${ }^{3}$, O. Daher ${ }^{3}$, P. Bodenmann', J.-P. Zellweger ${ }^{4}$

${ }^{1}$ Policlinique Médicale Universitaire, Lausanne, ${ }^{2} \mathrm{CHUV}$, Lausanne, ${ }^{3}$ Centre de Soins et Hôpital, Ste-Croix, ${ }^{4}$ Ligue

Pulmonaire Vaudoise, Lausanne

Background: Latent tuberculosis infection (LTBI) among asylum seekers is widespread and preventive treatment may avoid future cases of tuberculosis. The efficacy of screening and preventive treatment depends on the rate of completion of the treatment and varies from 10 to $86 \%$ according to various studies. The objective of this study was to assess the adherence to preventive treatment in a population of asylum seekers with latent tuberculosis infection recently arrived in Switzerland.

Methods: In a prospective cross - sectional study, asylum seekers recently arrived in two migration centers in the county of Vaud were screened with Interferon Gamma Release Assay (IGRA). Migrant with a positive test result were referred for a chest radiograph and a medical examination. After exclusion of individuals with active tuberculosis or prior therapy for tuberculosis, preventive treatment with rifampicine for 4 months (4R) or isoniazid for 9 months (9H) was offered to all participants except those with a contraindication. The adherence to treatment was assessed clinically during the monthly follow-up.

Results: From a population of 693 adults, 393 could be screened, and $98(24.93 \%)$ had a positive IGRA test. Eleven did not attend the initial medical examination. Among the 87 who were examined, eight received a full course of antituberculous therapy for documented or suspected active disease, 2 had already been treated for tuberculosis and $2 \mathrm{had}$ a contraindication to the administration of preventive treatment. A preventive treatment was offered to 75 individuals ( $4 \mathrm{R}$ in 74 and $9 \mathrm{H}$ in one), of which 59 (78.7\%) completed treatment. Thirteen (17.3\%) did not attend the monthly clinical control and two preventive treatments were suspended because of inadequate adherence (alcoholism and toxicomania).

Conclusions: Prescribing preventive treatment for latent tuberculosis to asylum seekers is a challenge. The vulnerability factors experienced by asylum seekers and the high mobility of this population make initial assessment of migrants with a positive test result and observance of the preventive treatment difficult but it seems possible to obtain a high rate of completion for migrants who accept the treatment. We believe that this is the result of the great level of coordination between nurses and medical practitioners and the efficacy of the management procedures.

\section{P111}

Treatment of MDR Tuberculosis in Switzerland. A Case Control Study

\section{P. Brun, H. Shang Meier, W. Karrer}

Luzerner Höhenklinik Montana, Crans-Montana

Background: There is growing concern about multidrug resistant (MDR) tuberculosis with resistance against isoniazid (INH) and rifampin (RMP). MDR tuberculosis can mostly be cured in western countries, but treatment with second-line drugs is difficult, long-lasting, provided with many side effects and expensive.

Methods: The aim of our study was to follow the patients with tuberculosis, hospitalized at the Luzerner Höhenklinik Montana (LHM) between 2005 and 2010, to evaluate how many of them were infected with multidrug resistant tuberculosis bacteria and to control their treatment to full recovery. The information in relation to post clinical treatment was gathered through personal contact with the treating physicians per e-mail and telephone interviews with the patients.

Results: Of 46 patients with tuberculosis hospitalized at the LHM between 2005 and 2010, five patients were assessed having multidrug resistant tuberculosis. Three suffered from pulmonary tuberculosis, one from tuberculous spinal infection (spondylodiscitis), and one from lymph node tuberculosis. One patient with lung tuberculosis was shown after some weeks of treatment not to have MDR tuberculosis, as in a second evaluation the laboratory findings proved susceptibility to all first line drugs. Drug therapy was performed with second line antituberculous drugs in combination of 4 to 5 drugs like Aminoglycosides, Ethionamid, Oxazolidinones and Quinolones. Drug therapy was given for at least 12 months. In two patients treatment had been completed before 2011, two are still under treatment. All patients required initially for a prolonged period intravenous drug therapy. In two patients surgery was performed during treatment (resection of the upper lobe of the lung, lymph node resection). All patients showed side-effects, such as nausea and vomiting and elevated liver enzymes, but also harmful complications occurred like labyrinthine hearing loss (aminoglycosides), polyneuropathy and neuropsychological abnormalities (Oxazolidinones).

Conclusion: Cases of multidrug resistant tuberculosis are still challenging for physicians and patients as diagnosis may not be evident and treatment remains difficult. In two cases the diagnosis was delayed due to different reasons. Duration of treatment is long, drug application is sometimes difficult as partially intravenous, side effects are frequent and especially the cost of treatment is remarkably high. 


\section{P112}

\section{Health-Related Quality of Life and Patient-Reported Outcome in Patients with Acute Bronchitis Receiving EPs ${ }^{\circledR} 7630$ Treatment}

H. Matthys' ${ }^{1}$, V.G. Lizogub ${ }^{2}$, P. Funk ${ }^{3}$, F.A. Malek ${ }^{3}$

${ }^{1}$ University Hospital Freiburg, Freiburg, D, ${ }^{2}$ National Medical University, Kiev, UA, ${ }^{3}$ Dr. Willmar Schwabe GmbH \& Co. KG, Karlsruhe, D

Background: Health-related quality of life (HRQL) and patientreported outcome (PRO) have become important target parameters for the evaluation of medical treatment within clinical trials. The herbal drug preparation EPs 7630 from the roots of Pelargonium sidoides (Umckaloabo ${ }^{\circledR}$; ISO Arzneimittel, Ettlingen, Germany) has been successfully used by adults and children $(>=1$ year of age) in the treatment of acute bronchitis for several years $[1,2]$. We present further exploratory results of a recently reported dose-finding study with $\mathrm{EPs}^{\otimes} 7630$ tablets in adults suffering from acute bronchitis [3], now focussing on HRQL and PRO.

Patients and Methods: 406 patients were randomly assigned to one of four parallel treatment groups (10, 20 or $30 \mathrm{mg} \mathrm{EPs} 7630$ tablets or placebo t.i.d.) for a treatment period of 7 days. HRQL and PRO were assessed by means of questionnaires (e.g. EQ-5D, EQ VAS, SF-12) as secondary outcome measures at each study visit or daily in the patient's diary.

Results: At day 7, patient-reported outcome measures were clinically relevantly and significantly more improved in all three $\mathrm{EPs}^{\circledast} 7630$ groups compared to placebo: remission or improvement of the 5 dimensions in EQ-5D ( $\mathrm{p}<0.05$, for all but two pair-wise comparisons between the active treatment groups and placebo in all 5 dimensions), EQ VAS ( $\mathrm{p}<0.0001$, each comparison), physical component score of SF-12 ( $\mathrm{p}<0.0001$, each comparison ), impact of patient's sickness ( $\mathrm{p}<0.05$, each comparison), shorter duration of activity limitation ( $\mathrm{p}<0.0001$, each comparison), patientreported treatment outcome $(\mathrm{p}<0.0001$, each comparison), and satisfaction with treatment ( $\mathrm{p}<0.0001$, each comparison).

Conclusion: The results indicate that treatment with EPs ${ }^{\circledast} 7630$ leads to a statistically significant and clinically relevant improvement of HRQL and PRO in adult patients with acute bronchitis in all three dosage levels investigated.

[1] Timmer A et al. Pelargonium sidoides extract for acute respiratory tract infections. Cochrane Database of Systematic Reviews 2008, Issue 3. Art. No.: CD006323. DOI:10.1002/14651858.CD006323.pub2.

[2] Agbabiaka TB et al. Pelargonium sidoides for acute bronchitis: a systematic review and meta-analysis. Phytomedicine 2008;15:378-85.

[3] Matthys $\mathrm{H}$ et al. Efficacy and tolerability of EPs 7630 tablets in patients with acute bronchitis: a randomised, double-blind, placebo-controlled dose-finding study with a herbal drug preparation from Pelargonium sidoides. Curr Med Res Opin 2010;26:1413-22.

\section{P113}

\section{Colonisation with Pseudomonas Aeruginosa and} Antibiotic Resistance Patterns in COPD Patients

\author{
K. Engler, K. Mühlemann, C. Garzoni, H. Pfahler, T. Geiser, \\ C. von Garnier
}

Inselspital, Bern

Background: P. aeruginosa infections play a major role in the exacerbation frequency and severity of COPD. Colonisation with P. aeruginosa accelerates lung function decline, most likely due to higher exacerbations frequencies. With this retrospective study, we aimed to determine epidemiologic and microbiological characteristics of colonisation with P. aeruginosa in COPD patients treated in a tertiary hospital centre.

Methods: Three-hundred and eighty-six patients that tested positive for P. aeruginosa in at least one respiratory sample during the study period (2004-2008) were retrospectively analysed for underlying respiratory disease, as well as colonisation patterns, morphology and antibiotic resistance patterns of $\mathrm{P}$. aeruginosa strains.

Results: Approximately one third $(n=112,29.0 \%)$ of patients with P. aeruginosa in respiratory samples suffered from COPD. Colonisation with P. aeruginosa was present in all COPD stages, but prevalence significantly increased with disease severity (GOLD 1: 4.7\%, GOLD 2: 10.3\%; GOLD 3: 10.8\%; GOLD 4: $17.9 \%$; $\mathrm{p}=0.0005)$. Forty-one percent of COPD patients with P. aeruginosa-positive respiratory samples were chronic carriers, of which $8 \%$ had mucoid strains. Carriage of a mucoid strain was associated with advanced COPD stage GOLD $4(\mathrm{p}=0.01)$. Resistance against newer generation cephalosporines was most frequently encountered and resistance against ciprofloxacin was found in more advanced COPD stages.

Conclusions: Colonisation with $\mathrm{P}$. aeruginosa was present in all COPD severity stages. Early diagnosis and treatment with appropriate i.v. anti-pseudomonas antibiotics may therefore be important in all COPD severity stages to prevent increasing resistance against ciprofloxacin, the only available oral anti-pseudomonas antibiotic.

\section{P114 \\ Efficacy of Roflumilast in Patients with a History of Frequent Exacerbations: Pooled Data from Pivotal 12-month Studies}

T.D. Bethke ${ }^{1}$, H. Rusch ${ }^{1}$, A. Gooss ${ }^{2}$

${ }^{1}$ Nycomed Deutschland GmbH, Konstanz, D, ${ }^{2}$ Nycomed Pharma AG, Dübendorf

Background: COPD exacerbations are associated with a substantial patient and healthcare burden, which is greater for frequent exacerbations. This analysis studied the effect of roflumilast on moderate or severe exacerbations by exacerbation history using pooled data from two 12-month studies.

Methods: M2-124 and M2-125 were replicate, randomised, placebo-controlled, double-blind, multicentre trials in COPD 
patients with severe-to-very severe airflow obstruction, a history of exacerbations and chronic bronchitis. Patients were randomised to roflumilast $500 \mu \mathrm{g}(\mathrm{n}=1537)$ or placebo once daily $(\mathrm{n}=1554)$ for 12 months.

Results: The rate of frequent exacerbations was lower with roflumilast than placebo: patients with 2 exacerbations: $11.5 \%$ with roflumilast vs $13.6 \%$ with placebo; with $3: 5.9 \%$ vs $6.8 \%$; with 4: $2.4 \%$ vs $3.5 \%$; with $5: 1.0 \%$ vs $2.1 \%$, and with $\geq 6: 0.6 \%$ vs $1.5 \%$. The mean exacerbation rate per patient per year was significantly lower with roflumilast than placebo in patients with $<2$ exacerbations in the previous year $(0.96$ vs $1.15,-16.5 \%$; $=0.006)$. The difference between roflumilast and placebo was greater in patients with $\geq 2$ exacerbations in the previous year ( 1.51 vs $1.95,-22.3 \%$; $\mathrm{p}=0.002$ ). A benefit was also seen with roflumilast vs placebo for time to first (hazard ratio [HR] $0.89, \mathrm{p}=0.019$ ), second (HR 0.79, $\mathrm{p}=0.001$ ) and third exacerbation (HR 0.73, p=0.003).

Conclusions: Frequency of exacerbations was lower and time to exacerbation was longer with roflumilast than placebo. The greatest benefit from roflumilast was seen in patients with a history of frequent exacerbations ( $\geq 2$ /year).

\section{P115 \\ Recovery of Lung Function After Intensive Treatment Due to Pulmonary Exacerbation in Children with Cystic Fibrosis}

\section{R. Kraemer, S. Gallati, M.H. Schoeni \\ Universitätsklinik für Kinderheilkunde, Bern}

Rationale: Lung function generally recovers after treatment of pulmonary exacerbations in patients with cystic fibrosis (CF). However, it is unclear how often return to previous baseline is achieved.

Objectives: (1) Determination of the proportion of children who significantly improved or failed to recover lung function after intensive treatment due to pulmonary exacerbation, and (2) identification of factors associated with success or failure over a substantial range of time.

Methods: Data from 24 CF-patients ( 15 males, 9 females) covering a time-span of 3 years around 44 occasions of hospitalization collected prior and after intensive treatment of pulmonary exacerbations were investigated. Serial measurements of whole-body plethysmography, multibreath nitrogen washouts and blood gas analysis provided data pertaining to functional residual capacity (FRCpleth, FRCMBNW), lung clearance index (LCI), volume of trapped gas (VTG), effective specific airway resistance (sReff), forced expiratory indices (FEV1, FEF50), $\mathrm{PaO} 2$ and $\mathrm{PaCO} 2$.

Results: Significant changes were observed for oxygenation given by $\mathrm{PaO} 2(\mathrm{p}<0.001)$, as well as sReff $(\mathrm{p}=0.032)$ and FEF50 $(\mathrm{p}=0.038)$ indicating an improvement of lung function, however, only after 24 occasions (54.5\%). During 13 occasions (29.5\%) lung function remained within a $\pm 8 \%$ change, and during 7 occasions (15.9\%) lung function even deteriorated.

Conclusions: Improvement of oxygenation depended specifically on improvement of obstruction (sReff) and small airway function (FEF50). In contrast, deterioration of gas exchange was closely associated with failure to recover ventilation inhomogene- ities (LCI) and/or to the decrease of trapped gas (VTG). In consequence, these complex physiopathological interactions regarding assessment of treatment efficacy demonstrate, that outcome parameters have to be carefully selected and defined, and not restricted to spirometric parameters only.

\section{P116 \\ Frequency of Prolonged Viral Shedding in Influenza A (H1N1) Infection of Lung Transplant Recipients}

M. Schuurmans, A. Zuercher, G.M. Tini, S. Gerber, C. Benden, A. Boehler

University Hospital of Zurich, Zurich

Background/Aim: Influenza A (H1N1) infection is considered a serious threat to lung transplant recipients (LTR) because immunosuppression may lead to potentially severe viral infection and possible bacterial superinfection both having the potential to trigger allograft rejection. Influenza vaccination is recommended for LTR. Prolonged viral shedding (PVS) after influenza infection is generally defined as positive detection of influenza A (H1N1) virus by real-time polymerase chain reaction (RT-PCR) at day 7 after diagnosis. The aim of this observational study was to quantify the viral shedding among laboratory-proven $\mathrm{H} 1 \mathrm{~N} 1$ infections among LTR in consecutive influenza seasons.

Methods: LTR routinely are offered influenza vaccination at follow-up visits in our outpatient clinic. LTR are also instructed to contact and visit our clinic when signs of infection occur or lung function deteriorates $>10 \%$ on consecutive days. Prompted by these events we routinely perform nasopharyngeal swabs (NPS) for viral and bacterial analysis. In case of suggestive symptoms for influenza infection we start oseltamivir (Tamiflu) and moxifloxacin (Avalox) treatment pending results from NPS. For laboratory proven $\mathrm{H} 1 \mathrm{~N} 1$ infection we continue both treatments until weekly performed NPS become negative. We defined PVS as laboratoryproven $\mathrm{H} 1 \mathrm{~N} 1$ infection with at least 2 consecutive positive RT-PCR results 7 or more days apart.

Results: In the influenza season 2009/2010 seven patients had H1N1 infection of which 2 had PVS (28\%). Two infections occurred in vaccinated LTR. All were managed on an outpatient basis without serious complications. For the influenza season 2010/2011 we have so far documented nine H1N1 infections with PVS in 5 LTR (55\%). All infected LTR had previously received at least 1 dose of $\mathrm{H} 1 \mathrm{~N} 1$ vaccine. Two infections required hospitalisation. 3 LTR had PVS for $>3$ weeks. Documentation is ongoing. Patient, treatment and vaccination characteristics will be provided.

Conclusion: PVS occurred in consecutive influenza seasons in $28 \%$ and $55 \%$ of all H1N1 infections in LTR. Infections occurred despite vaccination in 11 of 16 LTR. In this context early treatment resulted in favourable outcomes rarely requiring hospitalisation. 


\section{Poster Session 7 \\ Various Topics}

\section{P117 \\ Effects of Acute Exposure to Moderate Altitude on Vascular Function and Metabolism}

A.-C. Stoewhas ${ }^{1}$, T.D. Latshang ${ }^{1}$, C.M. Lo Cascio ${ }^{1}$, K. Stadelmann ${ }^{1}$, N. Tesler ${ }^{2}$, R. Huber ${ }^{2}$, P. Achermann ${ }^{1}$, K.E. Bloch ${ }^{1}$, M. Kohler ${ }^{1}$

${ }^{1}$ University Hospital Zurich, Zurich, ${ }^{2}$ Children's Hospital Zurich, Zurich

Background: Travel to moderate altitude for leisure activities or occupational reasons is popular. However, little is known about the effect of acute exposure to moderate altitude on vascular function and metabolism. Therefore, the purpose of our study was to investigate the effects of hypobaric hypoxia induced by acute altitude exposure on vascular function and metabolism in healthy volunteers.

Methods: In 51 healthy male subjects, oxygen saturation, blood pressure, heart rate, arterial stiffness (stiffness index, SI), lipid profiles and insulin resistance (HOMA-index) were measured at 490 $\mathrm{m}$ (Zurich) and at two consecutive days at moderate altitude (2590 m, Davos Jakobshorn).

Results: Mean (SD) age of the study population was 26.9 (9.3) years and mean BMI was $23.1(2.5) \mathrm{kg} / \mathrm{m}^{2}$. Overnight oxygen saturation decreased significantly upon ascent to $2590 \mathrm{~m}$. Mean blood pressure and heart rate were significantly increased at $2590 \mathrm{~m}$ compared to $490 \mathrm{~m}$ but this was not associated with an increase in arterial stiffness (table).

There was a significant increase in high density lipoprotein and a decrease of cholesterol/ high density lipoprotein ratio and triglycerides at altitude, however, insulin-resistance did not change significantly upon ascent to altitude (table).

Conclusion: Acute exposure to altitude is associated with considerable changes in blood pressure, heart rate and lipid profile, most likely due to increased sympathetic nervous system activity.
Our findings may be of relevance to patients with cardiovascular disease when traveling to altitude.

Grant support: Zurich Center for Integrative Human Physiology, SUVA

\section{P118}

\section{An Unusual Case of Severe Pulmonary Hypertension}

E. Lo Priore, T. Schmidhauser, M. Pons, A. Azzola

Ospedale Civico, Lugano

Background: Pulmonary hypertension $(\mathrm{PH})$ is a threatening condition of various origin commonly encountered in daily clinical practice, defined by a mean pulmonary artery pressure $>25$ $\mathrm{mmHg}$. $\mathrm{PH}$ is often secundary to left heart disease, pulmonary embolism (PE), or chronic hypoxic lung disease. Pulmonary arterial hypertension $(\mathrm{PAH})$ is caused by a paucity of rare diseases. The incidence of acute PE is around 1/1'000 persons/year and represents the third cardiovascular cause of death. PE secondary to malignancy is a common paraneoplastic manifestation. Hovewer direct seeding of cancerous microemboli to the pulmonary circulation is uncommon.

Case report: a 75-year-old caucasian man was admitted to our ward because of gradual onset of dyspnea over the last two months. The physical examination showed bilateral pitting edemas and bilateral diffuse crackles. The laboratory exames displayed significant elevation of the D-dimer value and marked sideropenic anemia. CT-scan excluded acute PE but showed a diffuse interstitial involvement of unclear etiology and dilatation of the pulmonary arteries. The presence of $\mathrm{PH}$ was confirmed by echocardiography which revealed severly increased systolic pulmonary artery pressure. The clinical condition worsened rapidly. Due to severe hypoxic respirature failure the patient required oro-tracheal intubation. Despite broad spectrum antibiotics, high dose corticosteroids and extensive support therapy, cardiac arrest and death occurred four days after ICU admittance. Autopsy was performed. The macroscopic lung examination showed an increase texture without thrombotic material in the main branch arteries, but the histological analysis revealed several neoplastic thromboemboli as well as a carcinomatous lymphagitis. The primary tumor was

Table 1. Effects of altitude exposure on vascular function and metabolism (for Abstract P117)

\begin{tabular}{|c|c|c|c|}
\hline Variable & $490 \mathrm{~m}$ & 2590 m (day 1) & $2590 \mathrm{~m}$ (day 2) \\
\hline Oxygen saturation (\%) & $95.9(1.0)$ & $89.7(1.8)^{* *}$ & $90.6(1.8)^{\star *} \# \#$ \\
\hline Mean blood pressure (mmHg) & $88.5(5.6)$ & $92.9(6.5)^{* *}$ & $94.2(6.0)^{* *}$ \\
\hline Heart rate $(\mathrm{bpm})$ & $56.7(8.8)$ & $60.1(10.4)^{*}$ & $60.1(9.5)^{* *}$ \\
\hline Stiffness Index (m/s) & $6.0(1.4)$ & $6.3(1.3)$ & $6.1(1.0)$ \\
\hline Total Cholesterol (mmol/l) & $4.0(0.7)$ & $4.1(0.7)$ & $4.04(0.8)$ \\
\hline HDL-Cholesterol (mmol/l) & $1.2(0.3)$ & $1.3(0.2)^{\star \star}$ & $1.4(0.3)^{\star *}$ \\
\hline Chol./HDL-Chol.-Ratio & $3.3(0.8)$ & $3.2(0.8)^{*}$ & $3.1(0.8)^{\star \star} \#$ \\
\hline LDL-Cholesterol (mmol/l) & $2.6(0.7)$ & $2.7(0.7)$ & $2.6(0.7)$ \\
\hline Triglycerides (mmol/l) & $0.8(0.3)$ & $0.7(0.3)^{*}$ & $0.7(0.3)^{\star *}$ \\
\hline HOMA-Index $(\mu \mathrm{U} / \mathrm{ml}) /(\mathrm{mmol} / \mathrm{l})$ & $0.7(0.5)$ & $0.7(0.5)$ & $0.6(0.3)$ \\
\hline
\end{tabular}

Values are means (SD). Chol.= cholesterol; HDL= high density lipoprotein; $\mathrm{LDL}=$ low density lipoprotein, $\mathrm{HOMA}-\mathrm{index}=\mathrm{Homeostatic}$ model assessmentindex $=$ fating insulin $(\mu \mathrm{U} / \mathrm{ml}) \times$ fasting glucose $(\mathrm{mmol} / \mathrm{l}) / 22.5{ }^{*} \mathrm{p}<0.05$ and ${ }^{* *} \mathrm{p}<0.001 \mathrm{vs} 490 \mathrm{~m}$; $\mathrm{p}<0.05$ and \#\# $\mathrm{p}<0.001 \mathrm{vs} 2590 \mathrm{~m}($ day 1$)($ ANOVA). 
identified as a poorly differentiated signet-ring cell gastric adenocarcinoma with multiple organ involvement (lymph nodes, liver, bone marrow).

Conclusions: Pulmonary tumor embolism (PTE) is described as possible first clinical manifestation of solid tumors, specially in case of occult gastric cancer. PTE_is often associated to severe pulmonary hypertension and commonly diagnosed only postmortem. PTE should be considered as possible cause of $\mathrm{PH}$, specially if absence of evidence for other causes and if other suspicious signs are present (i.e. interstitial lung disease possibliy due to lymphangitis or sideropenic anemia).

\section{P119 \\ Mountain Medicine Outpatient Consultation; Report of the Lausanne Experience}

P.-Y. Jayet, C. Sartori, E. Rexhaj, S. Rimoldi, H. Duplain, U. Scherrer

CHUV, Lausanne

Background: With development of modern transport facilities, travel to high-altitude destinations has become increasingly popular. Large numbers of persons who often do not have any previous high altitude experience are exposed to high-altitude related medical risks and seek advice. We recently opened a high-altitude medicine outpatient consultation staffed by experienced high-altitude medicine physicians with two aims in mind; 1) provide advice for persons planning to travel to high altitude with no previous high altitude experience, and 2) investigate and provide advice to patients who experienced high-altitude related medical problems during previous exposure. Here, we report the experience for 2010.

Methods: Patient work up consisted of a detailed medical history (including potential fetal and/or perinatal pathologic events and specific altitude related medical problems), complete clinical examination, and detailed study of the planned trek (ascension rate, sleeping altitude). Subjects with no previous high altitude experience planning treks implicating a possible risk of high-altitude pulmonary edema (HAPE) underwent assessment of the pulmonary artery pressure response (PAP, by echocardiography) to a hypoxic challenge in order to identify individuals at potential risk for this problem. A systolic PAP response $\geq 35 \mathrm{mmHg}$ during the hypoxic challenge $(\mathrm{FiO} 2,11.5)$ was considered indicating an increased risk for HAPE.

Results: In 2010, 51 persons were examined at our high altitude consultation (30 males, mean age 49 y, range, 22-72). 36\% were referred by their practitioners and $64 \%$ were self referrals. The majority of subjects (75\%) were seeking advice before high altitude exposure; roughly half of them suffered from a medical condition (cardiovascular, ORL or respiratory, and/or metabolic disease) requiring additional specific counseling. During the hypoxic challenge PAP increased from $25 \pm 11$ to $31 \pm 9 \mathrm{mmHg}$. In 10 of the subjects, PAP increased to a value $\geq 35 \mathrm{mmHg}$ putting these subjects at risk for HAPE. Based on the result of the examination, we strongly advised against high altitude exposure in 1 subject suffering from pulmonary hypertension; AMS or HAPE prophylaxis was prescribed in 9 and 7 subjects, respectively. None of the subjects reported major altitude-related medical problems during the trek.

Conclusion: These data demonstrate the need of specialized counseling for the steadily increasing number of people visiting high altitude destinations.

\section{P120 \\ Analgesic Intolerance in a 9-Year-Old Patient \\ C. Geidel, K.-H. Stirner, A. Jung, R. Lauener \\ Hochgebirgsklinik Davos, Davos}

Background: Aspirin-exacerbated respiratory disease (AERD) is an allergy-like reaction after intake of nonsteroidal anti-inflammatory drugs (NSAID). It shows no IgE-mediated mechanism, but an imbalance in arachidonic acid metabolism, which is enhanced by the acute intake of NSAID. The prevalence in the total population is estimated as 0.6 to $2,5 \%$. Epidemiological data suggests that $5-10 \%$ of all asthmatics do react with life-threatening asthma attacks after the ingestion of aspirin or other NSAID. In children $<10$ years, AERD is recognized as extremely rare. Here we report a 9 yrs old patient with proven AERD.

Case report: The first emergency hospitalization (paediatric ICU) occurred because the patient developed angioedema and dyspnoea after taking diclofenac $25 \mathrm{mg}$ in the context of a febrile infection. During the months before there already had been a similar situation after administration of diclofenac, which could be managed on outpatient basis. Medical history showes a normally developed boy. A single episode of seizure during fever was described as well as a cow's milk intolerance during infancy. Symptoms in terms of asthma, rhinitis, nasal polyps or urticaria weren't observed. Administration of paracetamol (doses up to $17 \mathrm{mg} / \mathrm{kg}$ ) during febrile infections were well tolerated. Allergy tests showed no sensitization, a prick-to-prick test with diclofenac was negative. There was high suspicion of an intolerance to analgetics as cause of the symptoms. The 'gold standard' of diagnosis, an aspirin challenge via inhalation (patient inhales rising doses of acetylsalicylic acid starting with $1,25 \mathrm{mg}$ ) showed a decrease of FEV1 to about $50 \%$ predicted after inhalation of $5 \mathrm{mg}$ acetylsalicylic acid, assuring the diagnosis of aspirin-exacerbated respiratory disease. Adaptive desactivation can induce a reversible tolerance to NSAID, which also leads to an improvement in signs and symptoms of an underlying AERD. This potential therapy wasn't considered in our patient for the moment because of his young age. The boy's medical history suggested paracetamol as a possible intervention in case of fever or pain. Alternatively, celecoxib might be considered for treatment.

Conclusion: Aspirin-exacerbated respiratory disease is a rare in children. However, in view of the possible dramatic course of the disease and frequently used NSAR in daily life this condition should not be underestimated. 


\section{P121}

\section{Recurrent Pulmonary Infections in an Elderly Woman}

D. Studer ${ }^{1}$, M. Gugger ${ }^{1}$, B. Klaeser ${ }^{1}$, M. Kaufmann ${ }^{2}$, R.A. Schmid ${ }^{1}$, T. Geiser ${ }^{1}$

${ }^{1}$ Inselspital, Bern, ${ }^{2}$ Pneumologische Praxis, Solothurn

Case presentation: An 81-year old, never-smoking woman presented herself with recurrent pulmonary infections treated with antibiotics. She complained about dyspnea on exertion, progressive dry cough, night sweating, heat feeling in the head, diarrhea and intermittently thoracic oppression on exertion. She had diabetes mellitus type 2, hypothyreosis due to thyreoiditis de Quervain and obesity. The physical findings presented a patient in a good general condition with unremarkable pulmonary auscultation. The lung function test revealed normal static and dynamic volumina.

In the CT scan a smooth right lower lobe nodule (size $3.5 \mathrm{~cm}$ ) with enlarged hilar lymph nodes was seen. Bronchoscopy showed an endoluminal tumor in the right lower lobe. Transbronchial needle aspiration showed a neuroendocrine tumor not differentiable between typical versus atypical tumor. Because of the low rate of proliferation (1-2\%) there was no evidence for an undifferentiated tumor. The measurement of the 24-hour urinary excretion of 5-hydroxyindoleacetic acid was normal. Octreotidscintigraphy revealed a somatostatin receptor-expressing nodule in the right lower lobe without any metastasis, indicating a carcinoid tumor. Magnetic resonance imaging of the cerebrum and sceletal scintigraphy ruled out any distant metastasis. Finally, a curative surgery of the resectable carcinoid was performed. Histopathologically, a typical carcinoid tumor was found without any lymphatic metastasis in 10 reviewed lymph nodes.

Conclusion: Bronchial carcinoid tumors are a rare group of pulmonary neoplasms mainly occurring in the fifth or sixth decade. We present a patient over 80 years old presenting with a typical carcinoid tumor that was finally diagnosed due to recurrent pulmonary infections.

\section{P122}

\section{Comparison of Different Brushing Techniques to} Obtain Nasal Epithelial Cells from Human Subjects

\author{
A. Stokes ${ }^{1}$, E. Kieninger ${ }^{1}$, L. Künzi' ${ }^{2}$, B.S. Kopf ', M. Geiser², \\ C. Casaulta ${ }^{1}$, N. Regamey ${ }^{1}$ \\ ${ }^{1}$ University Children's Hospital Berne, Berne, ${ }^{2}$ University of \\ Berne, Berne
}

Background: Nasal epithelial cells have been shown to be good surrogate markers for bronchial epithelial cells in inflammation studies. We aimed at comparing different available brushing techniques allowing collection of nasal epithelial cells with regard to efficiency in establishing cell cultures and acceptability to subjects, especially to children.

Methods: Nasal epithelial cells were obtained by brushing the inferior surface of the middle turbinate of both nostrils (each nostril was brushed twice) using three different brushing instruments: a 3-mm cytology brush, a neonatal flocked nasal swab and a nasal mucosal curette. Primary cell cultures were established by seeding freshly brushed nasal cells into growth medium. Cell count, cell viability (assessed by trypan blue staining), cell differentiation (assessed on cytospin preparations by hematoxylin and eosin staining) and success rate in establishing cell cultures (assessed by time to confluency) were compared between groups. A standard numeric pain intensity scale was used to assess the acceptability of each method to subjects.

Results: So far 10 subjects have been brushed, each of them by two different instruments. Higher number of cells were obtained using cytology brushes $\left(1.5 \times 10^{6} \pm 1.1 \times 10^{6}\right.$ cells $\left./ \mathrm{mL}\right)$ compared to nasal swabs $\left(0.8 \times 10^{6} \pm 0.7 \times 10^{6}\right.$ cells $\left./ \mathrm{mL}\right)$ and curettes $(0.8 \times$ $10^{6} \pm 0.6 \times 10^{6}$ cells $\left./ \mathrm{mL}\right)$. Viability was highest for cells obtained using cytology brushes $(92.5 \pm 5 \%)$ compared to the other two techniques (swabs: $61.0 \pm 30 \%$; curette: $62.6 \pm 30 \%$ ) (all $\mathrm{p}<0.05)$. More than $95 \%$ of cells obtained with all instruments were of epithelial origin. Success rate in establishing primary cell cultures (confluent cell layers after $10 \pm 4$ days in a T25 cell culture flask) was $>90 \%$ with cytology brushes and approximately $50 \%$ with the other techniques. Pain intensity was lowest with swabs and highest with brushes.

Conclusion: All brushing techniques allow collection and growth of primary human nasal epithelial cells. The most efficient but also most unpleasant method is the nasal cytology brush. These findings need to be considered when planning studies involving children. 


\section{P123 \\ Efficacy of Roflumilast When Used with Concomitant Inhaled Corticosteroids from the OPUS/RATIO Studies}

H. Rusch ${ }^{1}$, A. Gooss², T.D. Bethke', S. Rennard ${ }^{3}$

${ }^{1}$ Nycomed Deutschland GmbH, Konstanz, D, ${ }^{2}$ Nycomed Pharma AG, Dübendorf, ${ }^{3}$ University Nebraska Medical Center, Omaha, USA

Background: Roflumilast, an oral, selective phosphodiesterase 4 inhibitor, reduces exacerbation rates and improves lung function in COPD patients. Inhaled corticosteroids (ICS) are commonly used to manage COPD, particularly among individuals at risk for exacerbations. This post-hoc analysis studied the effects of roflumilast vs placebo in COPD patients with or without concomitant ICS treatment.

Methods: This pooled, post-hoc analysis of two replicate, randomized, double-blind, placebo-controlled studies (M2-111 and M2-112) compared oral roflumilast $500 \mu \mathrm{g}$ QD with placebo over 52 weeks. Stable dose ICS use was permitted. Lung function outcomes and exacerbation rates were examined.

Results: Of 2686 randomized patients, 1622 used concomitant ICS ( $n=809$ roflumilast and $n=813$ placebo) and 1064 did not ( $n=518$ and $n=546$, respectively). Among patients receiving concomitant ICS, $65 \%$ had severe disease vs $68 \%$ without ICS; $28 \%$ with ICS vs $21 \%$ without ICS had very severe COPD. For the overall population, pre-bronchodilator and post-broncho-dilator FEV1 improved (mean $\pm \mathrm{SE}$ ) with roflumilast compared to placebo by $51 \pm 7 \mathrm{~mL}(\mathrm{p}<0.0001)$ and $53 \pm 8 \mathrm{~mL}(\mathrm{p}<0.0001)$ and exacerba- tions were decreased by $14.3 \%(\mathrm{p}=0.026)$. With concomitant ICS use, pre-bronchodilator FEV1 $(53 \pm 9 \mathrm{~mL} ; \mathrm{p}<0.0001)$ and postbronchodilator FEV1 $(54 \pm 9 \mathrm{~mL} ; \mathrm{p}<0.0001)$ improved with roflumilast vs placebo. Without concomitant ICS use, pre-bronchodilator FEV1 $(49 \pm 13 \mathrm{~mL} ; \mathrm{p}=0.0002)$ and post-bronchodilator FEV1 $(53 \pm 13 \mathrm{~mL} ; \mathrm{p}<0.0001)$ improved with roflumilast compared to placebo. Roflumilast+ICS reduced the moderate/severe exacerbation rate vs placebo+ICS (rate ratio 0.72 vs 0.89 ; $-18.8 \%$; $\mathrm{p}=0.014$ ); the percentage of patients experiencing an exacerbation was numerically lower with roflumi-last+ICS vs placebo+ICS (36\% vs $42 \%$ ). Without concomitant ICS, the exacerbation rate (roflumilast 0.42 ; placebo $0.46 ;-7.7 \% ; \mathrm{p}=0.55$ ) was not affected by roflumilast; the percentage of patients experiencing an exacerbation was numerically lower with roflumilast vs placebo (23\% vs $27 \%$ ).

Conclusion: Roflumilast was effective at improving lung function independent of concomitant ICS use and reduced exacerbation rates in patients taking concomitant ICS, who may be recognized by clinicians as more likely to experience exacerbations. Roflumilast improves lung function, reduces the rate of exacerbations, and can have an additive effect in patients receiving concomitant ICS. 
Aubert, J.-D. P108

Achermann, E. P96

Achermann, P. 61, P117

Adler, D. 23, P92

Alves, M. 29

Andries, D. 60, P91

Azzola, A. 27, P118

Ballinary, P. 34

Barandun, J. 34

Barbe, L. 30, P79

Bastardot, F. 60, P91

Baty, F. 27, 63, 64, P72

Beckers, F. P83

Benden, C. 65, P86, P116

Berthod, G. P107

Bethke, T.D. P76, P114, P123

Betticher, D. 63, 64

Biaggi, P. P89

Blank, F. 32, P81, P82

Bloch, K.E. 59, 61, P88, P89, P90, P93P117

Bodenmann, P. P110

Boehler, A. 65, P85, P86, P100, P101, P116

Bonetti, A. P106

Bourqui, P. P94

Brändli, O. P96

Bratton, D. 58

Bridevaux, P.-O. P94

Brill, A.-K. P103, P104

Brun, P. P111

Brutsche, M.H. 27, 63, 64, P72, P78, P105

Bubendorf, L. 63

Buess, C. 34

Casaulta, C. P122

Cathomas, R. 64

Cesson, V. 31

Clarenbach, C.F. 25, 26, P71

Combescure, C. 62

Condrau, S. 27

Contal, O. 62

Craig, S. 58

Crowe, S. 63, 64

Daher, O. P110

Dalar, L. P86

DallaLana, K. P73

Davies, R.J.O. 58

Diemer, S. P74, P77

Dröge, C. 63,64
Dudler, J. P106

Dumont, P. P104

Duplain, H. P119

Edwards, M.R. 29

Egger, J.-M. P99

Engler, K. P113

Espa, F. 23, P92

Farr, P. P94

Fehr, T. 65, P93

Ferrarotti, I. 33

Fiechter Lienert, B. P100, P101

Fitting, J.W. P106

Fretz, G. P85, P86

Frey, M. P95, P96

Früh, M. 63, 64

Funk, P. P112

Furrer, M. P84

Gallati, S. P115

Garzoni, C. P113

Gazdhar, A. 30, P79, P80, P82

Geidel, C. 28, P74, P77, P120

Geiser, M. P122

Geiser, T. 29, 30, 32, P79, P80, P81, P82, P102, P103, P104, P113, P121

Gerber, S. P116

Gooss, A. P76, P114, P123

Grave de Peralta Menendez, R. P92

Guenat, O. 30, P79

Gugger, M. P80, P121

Haba-Rubio, J. 60, P87, P91

Hagemann, M. P104

Hanania, N. P76

Heinrichs, I. 28, P77

Heinzer, R. 60, P87, P91, P106

Henn, R.M. P88

Herren, T. P103

Hess, L. 59

Hofer, M. P100, P101

Holderegger, R. P99

Holt, P. 32

Huber, R. 61, P117

Huguenot, M. P81

Imboden, M. 33

Ionati, C. P109

Isenring, B.D. P72
Janssens, J.-P. 23, 62, P92, P94, P109

Jayet, P.-Y. P119

Jochmann, A. 24

Johnston, S.L. 29

Jolliet, P. 62

Jung, A. 28, P74, P77, P120

Kaelin, R. P96

Karrer, W. P95, P96, P111

Kaufmann, M. P121

Kern, L. 27, P72, P78, P105

Kieninger, E. 29, P122

Klaeser, B. P121

Klingler, K. 34

Kohler, M. 25, 26, 58, 59, 61, P71, P88, P89, P90, P93, P97, P117

Kolopp, L. P99

Kopf, B.S. 29, P122

Kraemer, R. 34, P115

Kuhn, M. P84

Kunz, A. P102

Künzi, L. P122

Kurowski, T. P100, P101

Lagler, U. P95

Langenskiold, E. P106, P110

Latshang, T.D. 59, 61, P88, P93, P117

Lauener, R. 28, P74, P77, P120

Lazor, R. P99, P106, P107

Leeson, P. 58

Letovanec, I. P107

Leuppi, D. 24

Lizogub, V.G. P112

Lo Cascio, C.M. 59, 61, P93, P117

Lo Priore, E. P118

Ludwig, C. P83

Ludwig, P. P104

Luisetti, M. 33

Lutchmaya-Flick, C. P110

Maier, S. 24

Malek, F.A. P112

Matthys, H. P112

Mazza Stalder, J. P107

Menz, G. 28

Mettler, G. 25, 26, P71

Meyenberger, C. P105

Michielin, O. P107

Miedinger, D. 24
Möller, A. 28

Morand, P. P83

Mueller, M.K. 65

Mühlemann, K. P113

Na, K.J. 63, 64

Namdar, M. P89

Neuhaus, G. P99

Nicod, L.P. 31, P108

Nicoll, D. 58

Ninet, B. P109

Noirez, L. P107

Nunn, A. 58

Nussbaumer-Ochsner, Y. P88

Obregon, C. 31

Ott, S.R. P102, P104

Ottaviani, S. 33

Pasche, A. P108

Pasquina, P. P94

Pépin, J.-L. 23, 62, P92

Pepperell, J.C.T. 58

Perret, E. P85

Perrig, S. 23, P92

Pfahler, H. P113

Pfister, A. P73

Pichler Hefti, J. P102

Piecyk, A. 34

Pless, M. 63, 64

Pons, M. P118

Prella, M. P108

Probst-Hensch, N.M. 33

Progin, I. P99

Puhan, M. P73, P96

Regamey, N. 29, P122

Rennard, S. P123

Rexhaj, E. P119

Rimoldi, S. P119

Ritscher, D. P96

Rochat, T. 33

Rodenstein, D. 23

Rodriguez, R. P105

Rothe, T. P75

Rotman, S. P108

Rüdiger, J. P78, P105

Rusch, H. P76, P114, P123

Russi, E.W. 25, 26, 33, P71, P89, P90 P97,

Sallin, P. 30

Sarivalasis, A. P110 
Sartori, C. P119

Sauthier, S. P109

Schaffroth, S. 24

Schawalder, N. P99

Scherer, T. 34

Scherr, A. 24

Scherrer, U. P119

Schmid, R.A. P121

Schmidhauser, T. P118

Schneider, T. P84

Schoeni, A. P79

Schoeni, M.H. P115

Schrenzel, J. P109

Schuurmans, M. 65, P85, P86, P116

Senn, O. 26, P73

Shang Meier, H. P111

Sistek, D. P108

Spaar, A. P96
Spertini, F. P106

Stadelmann, K. 61, P117

Steier, J. P97

Steurer-Stey, C. P73

Stirner, K.-H. P74, P120

Stoelben, E. P83

Stoewhas, A.-C. P90, P117

Stokes, A. P122

Stoller, S. P73

Stöwhas, A.-C. 25, 26, 61, P89

Stradling, J.R. 58, P89, P90

Strickland, D. 32

Stucki, A. P98

Studer, D. P121

Stumbles, P. 32

Susuri, N. P80

Tafti, M. 60, P91

Takahashi, H. 23
Tamm, M. 24, 27

Teschler, S. P71, P97

Tesler, N. 61, P117

Thun, G.A. 33

Thurnheer, R. 59

Tini, G.M. 65, P86, P116

Tobback, N. 60, P91

Turk, A. P96

Ulrich Somaini, S. P88

van der Feijst, Y. P98

van der Sluijs, K. 31

van Gestel, A.J.R. 25, 26, P71, P97

Vareille, M. 29

Vignaux, L. 62

Vogel, R. P82

Vollenweider, P. 60, P91 von Garnier, C. 32, P81, P82, P113

von Moos, R. 63

Wavre Oberhänsli, A. P87

Widmer, H.R. P82

Wiegand, J. 27

Zappa, F. 63, 64

Zellweger, J.-P. P110

Zorzetto, M. 33

Zuehlke, P. P95

Zuercher, A. P85, P116 